University of Tennessee Health Science Center

UTHSC Digital Commons

$12-2015$

\title{
Medicaid Discontinuity among Adults with Cardiovascular Disease or High Risk Conditions: Associations with Medication Adherence and Health Care Utilization
}

Kiraat Divyang Munshi

University of Tennessee Health Science Center

Follow this and additional works at: https://dc.uthsc.edu/dissertations

Part of the Health and Medical Administration Commons, Health Services Administration Commons, and the Health Services Research Commons

\section{Recommended Citation}

Munshi, Kiraat Divyang , "Medicaid Discontinuity among Adults with Cardiovascular Disease or High Risk Conditions: Associations with Medication Adherence and Health Care Utilization" (2015). Theses and Dissertations (ETD). Paper 397. http://dx.doi.org/10.21007/etd.cghs.2015.0220.

This Dissertation is brought to you for free and open access by the College of Graduate Health Sciences at UTHSC Digital Commons. It has been accepted for inclusion in Theses and Dissertations (ETD) by an authorized administrator of UTHSC Digital Commons. For more information, please contact jwelch30@uthsc.edu. 


\title{
Medicaid Discontinuity among Adults with Cardiovascular Disease or High Risk Conditions: Associations with Medication Adherence and Health Care Utilization
}

\author{
Abstract \\ BACKGROUND: Medicaid coverage among adults is often characterized by discontinuity - loss of \\ Medicaid coverage, and churning, or entering and exiting Medicaid - over short durations. Little is known \\ about the impact of having discontinuous Medicaid coverage on access to care, preventive care, \\ pharmacotherapy, primary care, and hospitalizations, among non-elderly adults with cardiovascular \\ disease (CVD) or high-risk conditions.
}

OBJECTIVES: This dissertation employed a three empirical research papers approach to pursue the following aims: (1) characterize the adult subpopulations with CVD or conditions placing them at high risk for CVD who lack continuous Medicaid coverage, and examine the characteristics associated with Medicaid discontinuity, (2) examine associations between Medicaid discontinuity, medication adherence and medication utilization, and (3) examine the associations between Medicaid discontinuity, access to care, preventive care, primary care visits, and hospitalizations.

METHODS: This was a retrospective comparative analysis of the 2002-2011 Medical Expenditure Panel Survey employing a repeated cross-sectional study design. Study sample included adults aged 18-64 years diagnosed with $\geq 1$ CVD (defined as acute myocardial infarction, coronary artery disease, congestive heart failure, peripheral and visceral atherosclerosis, or stroke) or high-risk conditions for CVD (defined as hypertension, lipid disorders, diabetes, or chronic kidney disease) who reported having Medicaid coverage any time during survey year. Individuals having continuous, full-year Medicaid coverage (Continuous Medicaid) were compared to those with(Discontinuous-Uninsured), and separately to those with(Discontinuous-Insured). Associations between Medicaid discontinuity, access to care, and preventive care were estimated using multivariate logistic regression. Medication adherence, measured as medication possession ratio (MPR) with adequate adherence being considered at MPR $>0.8$, was estimated using multivariate logistic regression. Medication utilization, measured as the number of allcause, and disease-specific prescription drug fills, was estimated using multivariate negative binomial regression. Four health care services utilization outcomes - inpatient, emergency room (ER), hospital outpatient, and office-based physician visits - measured as both, number of all-cause and number of disease-specific visits, were estimated using either zero-inflated negative binomial regression or negative binomial regression depending on the distribution of the outcome of interest.

RESULTS: Overall, $31.8 \%$ of adults with CVD or high-risk conditions for CVD hadcoverage, majority of whom (23.5\%) belonged to the Discontinuous- Uninsured group. Of those who had Medicaid at the beginning of the year, only $21.9 \%$ of the Discontinuous-Uninsured, and $8 \%$ of the Discontinuous-Insured still had Medicaid by the year end. Male gender, minority race/ethnicity, receiving disability benefits or participating in a federal assistance program, Medicaid managed care enrollment, and diagnosis of respiratory illnesses were the characteristics associated with lower odds of Medicaid discontinuity, whereas being married, residing in the South, having higher income, or education, being employed, and having fair to poor perceived health status were associated with higher odds of Medicaid discontinuity. Overall adherence to commonly prescribed therapeutic medication classes, measured as average MPR, was not significantly different between the Continuous Medicaid and the two discontinuous coverage groups, whereas examination of class-specific adherence yielded mixed results. Discontinuous Medicaid coverage was associated with significantly lower allcause and disease-specific prescription drug utilization among both the discontinuous Medicaid groups. Medicaid discontinuity was associated with poor access to care, and higher diseasespecific inpatient and ER hospitalizations among both the discontinuous Medicaid coverage groups. Additionally, among the Discontinuous-Uninsured, Medicaid discontinuity was associated with lower odds of routine medical checkup, lower all-cause primary care 
office visits, and higher disease-specific hospital outpatient visits.

CONCLUSION: Among non-elderly adults with CVD or high-risk conditions, for CVD having discontinuous Medicaid coverage was found to be associated with poor access to care and preventive care, poor adherence to certain medication classes and lower utilization of prescription medications, higher hospitalizations for CVD or associated conditions, and lower primary care office visits. Disruptions in and loss of Medicaid coverage among adults with CVD or high-risk conditions may lead to negative health outcomes due to the disruptions in continuity of care and inability to appropriately manage these disease conditions. This research provides strong support for implementation of policies to stabilize Medicaid coverage and reduce Medicaid discontinuity among individuals with CVD or high-risk conditions for CVD, such as the 12 month continuous Medicaid eligibility provisions currently in place for low-income children. Such policies will greatly improve some of the adverse access to care, preventive care, pharmacotherapy, and medical care outcomes observed in this study. Vulnerable populations with chronic, debilitating conditions may benefit from such policies to a greater extent compared to the overall low-income adult population. Simultaneously, reenrollment and outreach strategies may need to be more efficiently implemented to ensure individuals who are eligible for Medicaid, continue to remain enrolled in Medicaid. Such enabling strategies employed by Medicaid managed care organizations may be adopted by State Medicaid agencies to ensure greater continuity in Medicaid coverage for low-income vulnerable populations with chronic and debilitating diseases.

\section{Document Type}

Dissertation

Degree Name

Doctor of Philosophy (PhD)

\section{Program}

Health Outcomes and Policy Research

\section{Research Advisor}

James E. Bailey, M.D., M.P.H.

\section{Keywords}

Access to Care, Cardiovascular Disease, Health Care Utilization, Insurance Discontinuity, Medicaid, Medication Adherence

\section{Subject Categories}

Health and Medical Administration | Health Services Administration | Health Services Research | Medicine and Health Sciences | Public Health 
Medicaid Discontinuity among Adults with Cardiovascular Disease or High Risk Conditions: Associations with Medication Adherence and Health Care Utilization

\author{
A Dissertation \\ Presented for \\ The Graduate Studies Council \\ The University of Tennessee
}

In Partial Fulfillment

Of the Requirements for the Degree

Doctor of Philosophy

From The University of Tennessee

By

Kiraat Divyang Munshi

December 2015 
Copyright $(\underset{2}{ } 2015$ by Kiraat Divyang Munshi. All rights reserved. 


\section{DEDICATION}

To the Almighty for the invisible hand that has guided me throughout my life.

To my late grandparents for their blessings.

To my family for their love and unwavering support.

To my extended family and friends and the unsung heroes who have helped me in life.

To all the Average Joes and Janes out there. 


\section{ACKNOWLEDGEMENTS}

I have been lucky to have had exceptional dissertation committee members. I begin my expressing my deepest gratitude to my advisor and mentor, Dr. Jim Bailey, the most kind hearted, gentle person, and researcher and academic par excellence. I will cherish the time I have spent as your mentee for the rest of my life. The academic and research freedom you provide to your mentees is unparalleled, and the intellectually stimulating discussions regarding work and research, and conversations about all things non-academic have benefitted me immensely. Dr. Rob Nolly has been supportive throughout the doctoral program during the seminars and provided great feedback and inputs during discussions regarding this, and various other research studies. Dr. George Relyea has provided excellent guidance in the data analysis aspect of not only this dissertation, but other projects as well. Dr. Jim Wan has been kind to meet me several times on short notice and provide tremendous help in the study design and analytic methodology. Dr. Cameron Kaplan has always asked thought-provoking questions and provided insightful feedback that has helped me in critical thinking and improving my research studies. Thank you, y'all! I also thank all my teachers and professors for all the knowledge and wisdom I have gained during my educational pursuits.

I have been blessed to be born as the son of the most amazing, inspirational parents one can have in Mr. Divyang Munshi and Mrs. Varada Munshi. The tremendous upbringing I have had the fortune of having, the good values, beliefs, and morals infused in me have made me a better person and for that I am forever indebted to them. My best friend and wife, Satya Surbhi, has been the bedrock of my adult life. She has steadfastly been by my side through the all the highs and lows, and not only provided the love, affection and emotional support I needed, but being a colleague, has been my go-to person for all academic and career discussions. I thank my brother Killol and his wife Prutha for their love and support at all times in life, and especially during my doctoral education. I would like to thank my wonderful in-laws, Dr. A. K. Chaudhary and Mrs. Meena Chaudhary, for their unconditional love and support, and blessings during this critical phase in my life. I thank my brother-in-law, Dr. Bipul Baibhav, for his knowledgeable inputs for this dissertation in his capacity as a brilliant cardiologist, and thank him and his wife, Anna Steltenpohl, for their support and affection. I pay my respects to my beloved paternal and maternal grandparents, Uvaba (Smt. Urvashiben Munshi), Baaji (Mrs. Sangmitra Mehta), Dadaji (Mr. Rohit J. Mehta), and Dada (Mr. Jayendraprasad Munshi) whose blessings have made this achievement possible, and who I know are smiling in heaven.

I would also like to thank the SafeMed team, especially Jill Nault, Ms. Celia Ridley, and Bonnie Binkley. A big thank you to my friends, both in India and the US who have helped me in every way possible, and with whom I have shared some of the best moments of my life. I thank my colleagues in my doctoral program, especially Dr. Mustafa Hussein for his help with a part of this dissertation. And finally, with folded hands, I bow my head to Bhagavan for all that has been bestowed upon me in life. I am truly humbled! 


\begin{abstract}
BACKGROUND: Medicaid coverage among adults is often characterized by discontinuity - loss of Medicaid coverage, and churning, or entering and exiting Medicaid - over short durations. Little is known about the impact of having discontinuous Medicaid coverage on access to care, preventive care, pharmacotherapy, primary care, and hospitalizations, among non-elderly adults with cardiovascular disease (CVD) or high-risk conditions.

OBJECTIVES: This dissertation employed a three empirical research papers approach to pursue the following aims: (1) characterize the adult subpopulations with CVD or conditions placing them at high risk for CVD who lack continuous Medicaid coverage, and examine the characteristics associated with Medicaid discontinuity, (2) examine associations between Medicaid discontinuity, medication adherence and medication utilization, and (3) examine the associations between Medicaid discontinuity, access to care, preventive care, primary care visits, and hospitalizations.
\end{abstract}

METHODS: This was a retrospective comparative analysis of the 2002-2011 Medical Expenditure Panel Survey employing a repeated cross-sectional study design. Study sample included adults aged 18-64 years diagnosed with $\geq 1$ CVD (defined as acute myocardial infarction, coronary artery disease, congestive heart failure, peripheral and visceral atherosclerosis, or stroke) or high-risk conditions for CVD (defined as hypertension, lipid disorders, diabetes, or chronic kidney disease) who reported having Medicaid coverage any time during survey year. Individuals having continuous, full-year Medicaid coverage (Continuous Medicaid) were compared to those with $<12$ months of Medicaid coverage with no other insurance reported during the year (DiscontinuousUninsured), and separately to those with $<12$ months of Medicaid along with other sources of health insurance during the year (Discontinuous-Insured). Associations between Medicaid discontinuity, access to care, and preventive care were estimated using multivariate logistic regression. Medication adherence, measured as medication possession ratio (MPR) with adequate adherence being considered at $\mathrm{MPR} \geq 0.8$, was estimated using multivariate logistic regression. Medication utilization, measured as the number of all-cause, and disease-specific prescription drug fills, was estimated using multivariate negative binomial regression. Four health care services utilization outcomes - inpatient, emergency room (ER), hospital outpatient, and office-based physician visits measured as both, number of all-cause and number of disease-specific visits, were estimated using either zero-inflated negative binomial regression or negative binomial regression depending on the distribution of the outcome of interest.

RESULTS: Overall, $31.8 \%$ of adults with CVD or high-risk conditions for CVD had $<12$ months of Medicaid coverage, majority of whom (23.5\%) belonged to the Discontinuous-Uninsured group. Of those who had Medicaid at the beginning of the year, only $21.9 \%$ of the Discontinuous-Uninsured, and $8 \%$ of the Discontinuous-Insured still had Medicaid by the year end. Male gender, minority race/ethnicity, receiving disability benefits or participating in a federal assistance program, Medicaid managed 
care enrollment, and diagnosis of respiratory illnesses were the characteristics associated with lower odds of Medicaid discontinuity, whereas being married, residing in the South, having higher income, or education, being employed, and having fair to poor perceived health status were associated with higher odds of Medicaid discontinuity. Overall adherence to commonly prescribed therapeutic medication classes, measured as average MPR, was not significantly different between the Continuous Medicaid and the two discontinuous coverage groups, whereas examination of class-specific adherence yielded mixed results. Discontinuous Medicaid coverage was associated with significantly lower all-cause and disease-specific prescription drug utilization among both the discontinuous Medicaid groups. Medicaid discontinuity was associated with poor access to care, and higher disease-specific inpatient and ER hospitalizations among both the discontinuous Medicaid coverage groups. Additionally, among the Discontinuous-Uninsured, Medicaid discontinuity was associated with lower odds of routine medical checkup, lower all-cause primary care office visits, and higher disease-specific hospital outpatient visits.

CONCLUSION: Among non-elderly adults with CVD or high-risk conditions, for CVD having discontinuous Medicaid coverage was found to be associated with poor access to care and preventive care, poor adherence to certain medication classes and lower utilization of prescription medications, higher hospitalizations for CVD or associated conditions, and lower primary care office visits. Disruptions in and loss of Medicaid coverage among adults with CVD or high-risk conditions may lead to negative health outcomes due to the disruptions in continuity of care and inability to appropriately manage these disease conditions. This research provides strong support for implementation of policies to stabilize Medicaid coverage and reduce Medicaid discontinuity among individuals with CVD or high-risk conditions for CVD, such as the 12 month continuous Medicaid eligibility provisions currently in place for low-income children. Such policies will greatly improve some of the adverse access to care, preventive care, pharmacotherapy, and medical care outcomes observed in this study. Vulnerable populations with chronic, debilitating conditions may benefit from such policies to a greater extent compared to the overall low-income adult population. Simultaneously, reenrollment and outreach strategies may need to be more efficiently implemented to ensure individuals who are eligible for Medicaid, continue to remain enrolled in Medicaid. Such enabling strategies employed by Medicaid managed care organizations may be adopted by State Medicaid agencies to ensure greater continuity in Medicaid coverage for low-income vulnerable populations with chronic and debilitating diseases. 


\section{TABLE OF CONTENTS}

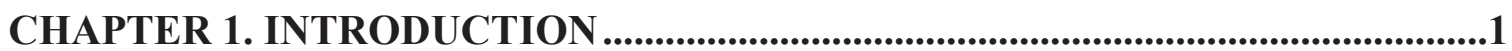

INSTABILITY IN HEALTH INSURANCE .......................................................

INSTABILITY IN MEDICAID AMONG LOW-INCOME ADULT

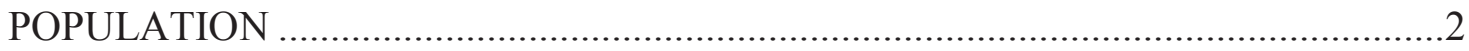

CARDIOVASCULAR DISEASE OR ASSOCIATED HIGH-RISK CONDITIONS

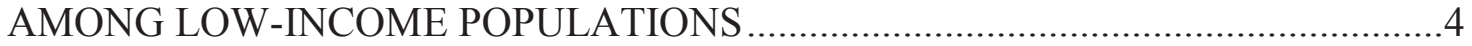

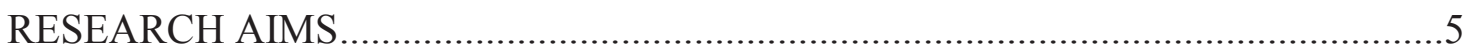

Aim 1: To Examine the Individual Characteristics Associated with Medicaid

Discontinuity ........................................................................................... 5

Aim 2: To Examine the Associations between Medicaid Discontinuity, Medication Adherence and Medication Utilization ..................................................5

Aim 3: To Examine the Associations between Medicaid Discontinuity, Access to

Care, Preventive Services Use, and Utilization of Health Care Services ...................5

\section{CHAPTER 2. CHARACTERISTICS ASSOCIATED WITH MEDICAID} DISCONTINUITY IN ADULTS WITH CARDIOVASCULAR DISEASE OR ASSOCIATED HIGH-RISK CONDITIONS ........................................................7

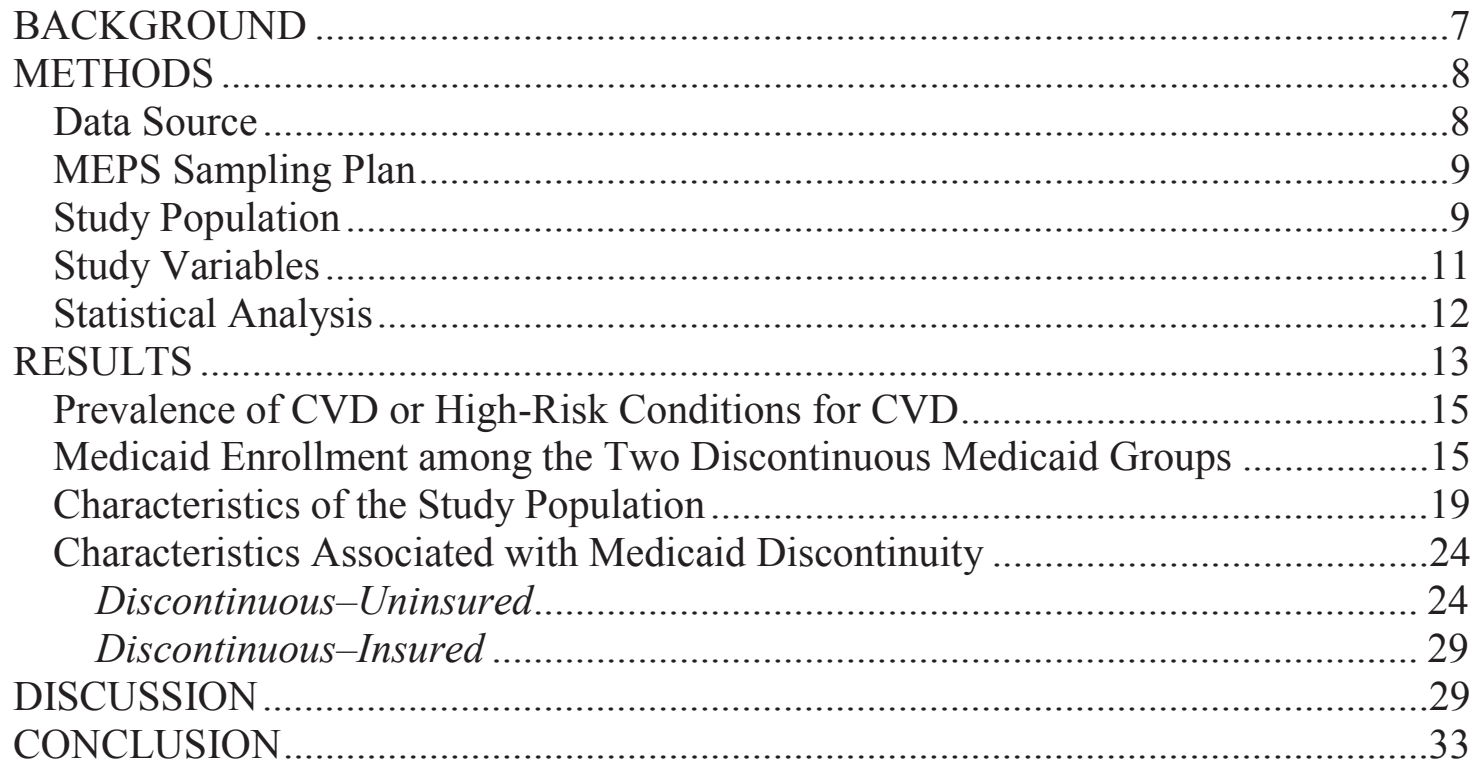

CHAPTER 3. ASSOCIATIONS BETWEEN MEDICAID DISCONTINUITY, MEDICATION ADHERENCE AND MEDICATION UTILIZATION AMONG ADULTS WITH CARDIOVASCULAR DISEASE OR ASSOCIATED HIGHRISK CONDITIONS ............................................................................................34

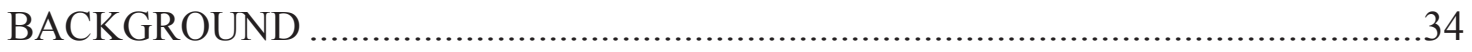

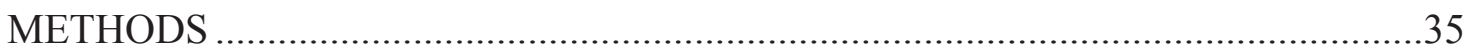

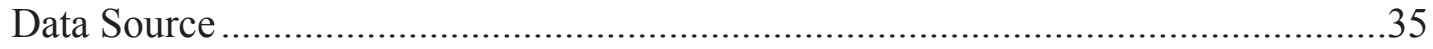

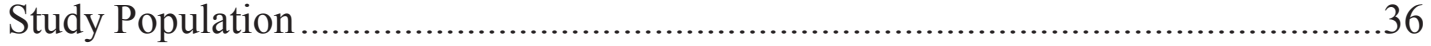

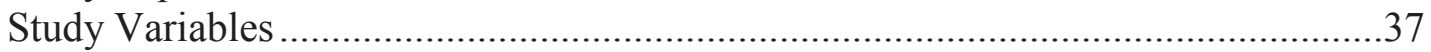




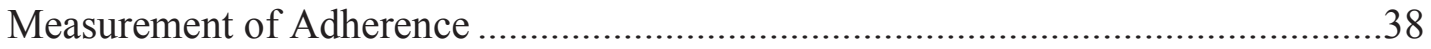

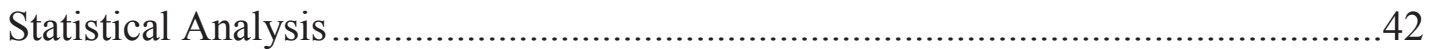

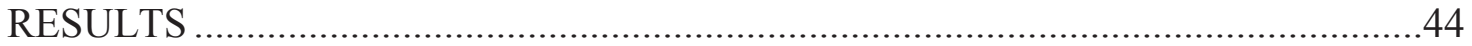

Characteristics of the Study Populations ...............................................................4

Prescription Medication Use in the Study Population ........................................53

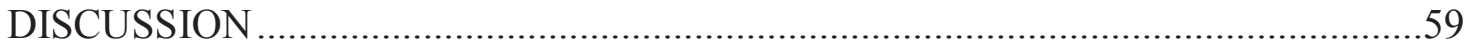

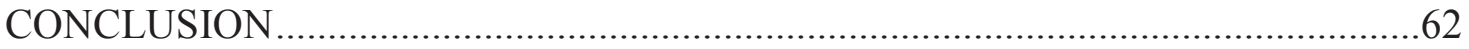

\section{CHAPTER 4. ASSOCIATIONS BETWEEN MEDICAID DISCONTINUITY, ACCESS TO CARE, PREVENTIVE CARE, AND HEALTH SERVICES UTILIZATION AMONG ADULTS WITH CARDIOVASCULAR DISEASE OR ASSOCIATED HIGH-RISK CONDITIONS ...............................................64}

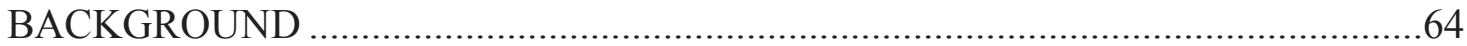

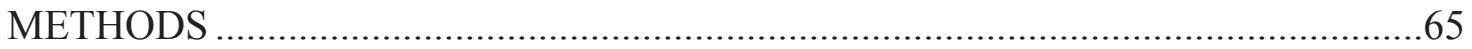

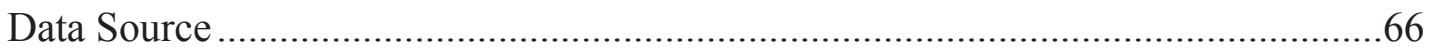

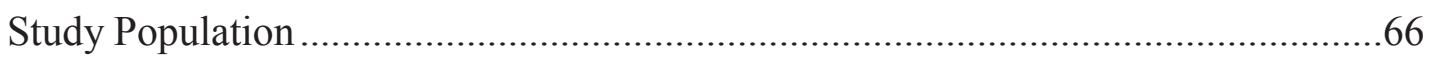

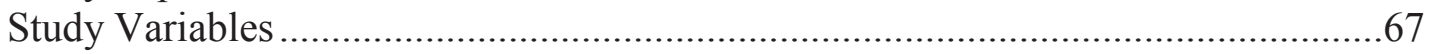

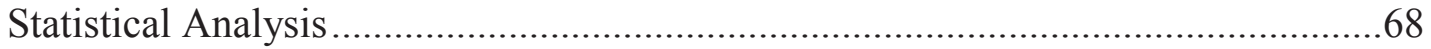

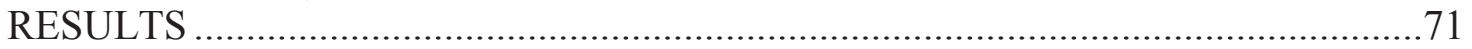

Characteristics of the Study Populations .............................................................. 71

Medicaid Enrollment among the Discontinuous Medicaid Coverage Groups ..........75

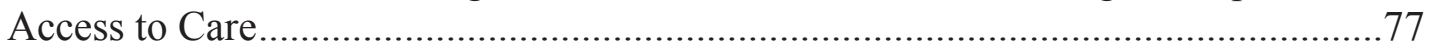

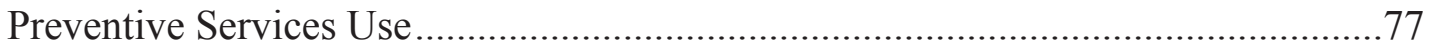

Utilization of Health Care Services ................................................................... 80

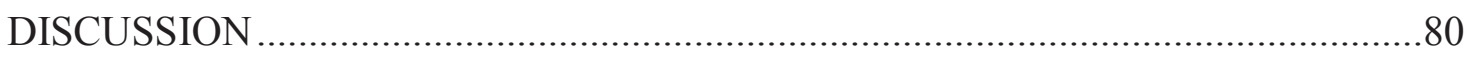

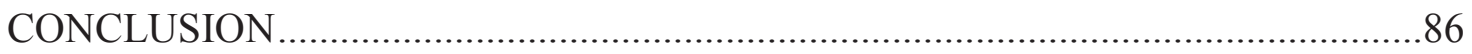

CHAPTER 5. CONCLUSION ...................................................................................88

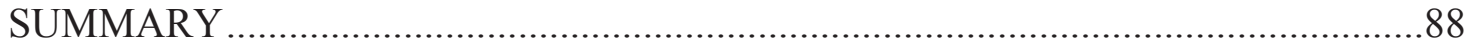

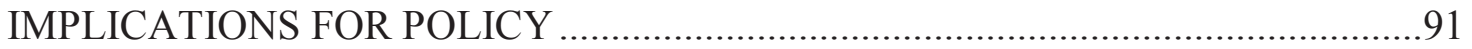

IMPLICATIONS FOR FUTURE RESEARCH ..................................................92

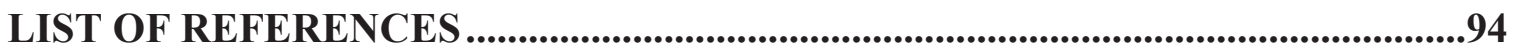

APPENDIX A. SUPPLEMENTAL MATERIALS FOR CHAPTER 3....................103

APPENDIX B. SUPPLEMENTAL MATERIALS FOR CHAPTER 4....................109

VITA 


\section{LIST OF TABLES}

Table 2-1. Diagnosis and Event Components Used to Select the Study Population......16

Table 2-2. Prevalence of CVD or High-Risk Conditions for CVD in the Study

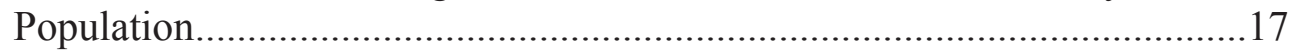

Table 2-3. Medicaid Enrollment among the Discontinuous Medicaid Groups..............18

Table 2-4. Characteristics of the Study Population ....................................................20

Table 2-5. Characteristics Associated with Medicaid Discontinuity in the Study

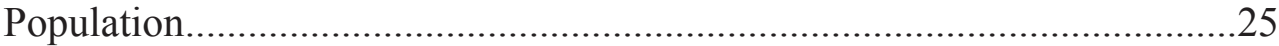

Table 3-1. Refill Days of Supply Algorithms and MPR Validation in 2011 Sample $(\mathrm{N}=5,475)$

Table 3-2. Refill Days of Supply Algorithms and MPR Validation in 2010 Sample $(\mathrm{N}=5,058)$

Table 3-3. Study Population Characteristics when Examining Medication

Adherence

Table 3-4. Study Population Characteristics when Examining Medication Utilization

Table 3-5. Summary Statistics for Prescription Medication Use in the Study

Population

Table 3-6. Association between Medicaid Discontinuity and Prescription

Medication Adherence in the Study Population....

Table 3-7. Association between Medicaid Discontinuity and Prescription Medication Utilization in the Study Population 58

Table 4-1. Characteristics of the Study Population (2002-2011) .72

Table 4-2. $\quad$ Patterns of Medicaid Coverage among Discontinuous Medicaid Groups ....76

Table 4-3. Association between Medicaid Discontinuity and Access to Care in the Study Population .78

Table 4-4. Associations between Medicaid Discontinuity and Preventive Services Use in the Study Population.

Table 4-5. Associations between Medicaid Discontinuity and Health Care Resources Utilization in the Study Population.... 
Table A-1. Characteristics Associated with Medication Adherence...........................105 


\section{LIST OF FIGURES}

Figure 2-1. MEPS Overlapping Panel Design .............................................................10

Figure 2-2. Schematic Representation of the Study Sample Derivation Process .............14

Figure A-1. Distribution of All-Cause Prescription Drug Fills among Continuous Medicaid and Discontinuous-Uninsured ...................................................103

Figure A-2. Distribution of All-Cause Prescription Drug Fills among Continuous Medicaid and Discontinuous-Insured

Figure A-3. Distribution of Disease-Specific Prescription Drug Fills among Continuous Medicaid and Discontinuous-Uninsured.

Figure A-4. Distribution of Disease-Specific Prescription Drug Fills among Continuous Medicaid and Discontinuous-Insured

Figure B-1. Distribution of All-Cause Inpatient Visits among Continuous Medicaid and Discontinuous-Uninsured

Figure B-2. Distribution of All-Cause Inpatient Visits among Continuous Medicaid and Discontinuous-Insured

Figure B-3. Distribution of Disease-Specific Inpatient Visits among Continuous Medicaid and Discontinuous-Uninsured

Figure B-4. Distribution of Disease-Specific Inpatient Visits among Continuous Medicaid and Discontinuous-Insured

Figure B-5. Distribution of All-Cause Emergency Room Visits among Continuous Medicaid and Discontinuous-Uninsured

Figure B-6. Distribution of All-Cause Emergency Room Visits among Continuous Medicaid and Discontinuous-Insured

Figure B-7. Distribution of Disease-Specific Emergency Room Visits among Continuous Medicaid and Discontinuous-Uninsured.

Figure B-8. Distribution of Disease-Specific Emergency Room Visits among Continuous Medicaid and Discontinuous-Insured

Figure B-9. Distribution of All-Cause Hospital Outpatient Visits among Continuous Medicaid and Discontinuous-Uninsured

Figure B-10. Distribution of All-Cause Hospital Outpatient Visits among Continuous Medicaid and Discontinuous-Insured 
Figure B-11. Distribution of Disease-Specific Hospital Outpatient Visits among Continuous Medicaid and Discontinuous-Uninsured.

Figure B-12. Distribution of Disease-Specific Hospital Outpatient Visits among Continuous Medicaid and Discontinuous-Insured

Figure B-13. Distribution of All-Cause Physician Office Visits among Continuous Medicaid and Discontinuous-Uninsured

Figure B-14. Distribution of All-Cause Physician Office Visits among Continuous Medicaid and Discontinuous-Insured

Figure B-15. Distribution of Disease-Specific Physician Office Visits among Continuous Medicaid and Discontinuous-Uninsured

Figure B-16. Distribution of Disease-Specific Physician Office Visits among Continuous Medicaid and Discontinuous-Insured 


\section{LIST OF ABBREVIATIONS}

$\mathrm{ACE}$

AHRQ

AIDS

ARB

CAD

CCC

CCI

$\mathrm{CHF}$

CHIP

CI

CKD

CMS

CVD

ED

ER

FPL

GED

$\mathrm{HC}$

HIV

HTN

IRR

$\mathrm{MCO}$
Angiotensin converting enzyme inhibitors

Agency for Healthcare Research and Quality

Acquired immunodeficiency syndrome

Angiotensin II receptor blockers

Coronary artery disease

Clinical classification code

Charlson Comorbidity Index

Congestive heart failure

Children's Health Insurance Program

Confidence interval

Chronic kidney disease

Centers for Medicare and Medicaid

Cardiovascular disease/cardiovascular diseases

Emergency department

Emergency room

Federal poverty level

General Educational Development

Household Component

Human immunodeficiency virus infection

Hypertension

Incidence Rate Ratio

Managed care organization 
MEPS

MPR

MSA

NCHS

OR

PDC

PPACA

PVA

SE

SSI

TANF

WHO
Medical Expenditure Panel Survey

Medication Possession Ratio

Metropolitan Statistical Area

National Center for Health Statistics

Odds ratio

Proportion of days covered

Patient Protection and Affordable Care Act

Peripheral vascular disease

Standard error

Supplemental Security Income

Temporary Assistance for Needy Families

World Health Organization 


\section{CHAPTER 1. INTRODUCTION}

\section{INSTABILITY IN HEALTH INSURANCE}

The health care system in the United States (U.S.) has widely recognized to be "fragmented". ${ }^{1}$ The systemic misalignment of incentives, and lack of coordination between physician, and hospitals, leading to poor care coordination, inefficient allocation of resources, contribute to this narrative. This in turn greatly hampers the quality of care that patients receive. Likewise, the health insurance system in the U.S. too is fragmented with most individuals covered by employer-sponsored private health insurance (48\%), and the poor, elderly, and disabled population groups being covered by governmentsponsored public health insurance (roughly $31 \%$ ), with the remaining either purchasing individual private health plans (just $5 \%$ of the total population), or remaining uninsured, which estimates peg at $15 \%$ of the total population, roughly the same as those on Medicaid and Medicare. ${ }^{2}$ Rules and regulations are set separately for both private health insurance by employers, and for public health insurance by government, regarding eligibility for the insurance plans paid for, the specific covered benefits and cost sharing.

The principal requirement for employer-sponsored insurance is employment. Losing employment, changing jobs, moving to different geographic regions, and starting one's own venture, are circumstances that can lead to losing employer-sponsored insurance coverage or gaps in insurance coverage. The number of individuals that lose insurance resulting from loss of employment increased significantly during times of economic recession. ${ }^{3,4}$ As a cost-cutting measure, employers may change the benefit structure of the plans by requiring their employees to pay more through higher premiums, deductibles or co-pays, and also by cutting the numbers and/or types of services covered. Such measures may make health insurance expensive for low-income employees and those who cannot afford such plan structures. When an individual employee receives employment-sponsored coverage, his/her entire family is covered under the insurance plan. Thus, employee spouses and children of may also experience insurance instability as well due to circumstances such as changes in benefit structure where spouse coverage is dropped, loss of coverage or employment, retirement, death or other circumstances.

Government-sponsored public health insurance too has its own set of eligibility criteria and enrollment procedures, and requires individuals to be aware of them to determine whether they are eligible or not, and if they are, to provide the appropriate documentation to enroll for these insurance plans, and maintain eligibility; this is especially true for Medicaid and Children's Health Insurance Plan (CHIP) that are also referred to as means-tested public health insurance programs. ${ }^{5}$ The eligibility requirements and enrollment procedures for Medicaid and CHIP differ from state-tostate, and so any change in residence across states may lead to loss of insurance coverage resulting from either ineligibility or failure to enroll successfully. Traditionally, before the passage of the 2010 Patient Protection and Affordable Care Act (PPACA), not only did the income eligibility thresholds vary greatly between the states, but they were also different for different population groups, such as more generous coverage limits for 
children and pregnant women, less generous coverage limits for non-elderly adults with children, and no coverage for non-elderly adults without any children. ${ }^{6}$ Small fluctuations in income levels for covered non-elderly adults would end up disqualifying individuals with incomes at or near eligibility thresholds. As with employer-sponsored health insurance, changes or loss in Medicaid coverage for one member of the family impacted the continuity in Medicaid for the entire family. Also, changes in family size and compositions would also impact eligibility for these public insurance programs, since yearly poverty levels that are established take into account these factors. The rules and regulations for eligibility and enrollment between public and private health insurance are different, and therefore, individuals who have to transition between one system to another also experience gaps in insurance coverage, either due to failure to follow enrollment procedures, or being unaware of such an option, or even due to the stigma of enrolling for Medicaid or other low-income public programs. ${ }^{6}$ It is broadly due to these diverse factors in this fragmented health insurance system, that individuals and families experience insurance instability and continue to swing into and out of various types of health insurance coverage.

\section{INSTABILITY IN MEDICAID AMONG LOW-INCOME ADULT POPULATION}

Medicaid, the nation's primary public health insurance program for the lowincome population, provides much-needed health insurance coverage to an estimated 67 million individuals who meet the eligibility criteria, which include having incomes and assets below a certain threshold level determined by each State. ${ }^{7}$ These include lowincome children, adults, elderly, and disabled who do not have access to private health insurance, and many of whom are not eligible for any other source of coverage. Medicaid however, has been described as a leaky sieve. Inefficient administrative hurdles and ponderous periodic paperwork requirements, result in discontinuity in Medicaid coverage, characterized by either loss of coverage or "churning" - frequent transitions into and out of various sources of coverage. ${ }^{8,9}$ Such instability often plagues Medicaideligible individuals, leading to both gaps in coverage and interruptions in the continuity of care. Families may have to find new providers or change their existing health treatments due to different provider networks or different services covered in their new health plans, or may end up forgoing needed care after becoming uninsured. Some research has been conducted to examine Medicaid instability and its impact on various outcomes, although the research in this area is not exhaustive.

Harman and colleagues examined Utah Medicaid claims data from 1990-1994 to study the impact of Medicaid interruptions on inpatient psychiatric services use. They found that interruptions in Medicaid were associated with an average of 0.63 more psychiatric hospitalizations per beneficiary, and 8.3 more psychiatric hospitalization days over the study period. ${ }^{10}$ Using the Ohio Medicaid claims from 1992-1999 Koroukian examined the impact of length of enrollment in Medicaid on use of screening mammography among women aged 40-64 years. They found that with each additional year of Medicaid enrollment, the proportion of women receiving screening mammography increased significantly, and [Odds Ratio (OR): 1.59; 95\% Confidence 
Interval (CI): 1.57-1.60], and the mean number of annual mammography exams increased from 0.08/year among women enrolled for less than a year to 0.26 /year among women enrolled for more than 7 years. ${ }^{11}$ Continuous Medicaid enrollment also was shown to have a positive impact on cervical cancer prevention. ${ }^{12}$ Harman and colleagues analyzed Florida Medicaid claims data from 1999-2002 to examine the impact of Medicaid interruption on depressed beneficiaries' health care utilization and expenditures. They found that inpatient episodes, the length of inpatient stay, number of emergency department (ED) visits, and total Medicaid expenditures increased significantly $(P<0.001)$ in the three-month period after an interruption in coverage of more than one month, indicating a significant negative impact of Medicaid instability among low-income elderly with mental illness. ${ }^{13}$ More recently, studies that have examined the impact of interruptions in Medicaid using state-specific data have found that instability in Medicaid is associated with higher risk for hospitalization for ambulatory care-sensitive conditions, and increase in overall program expenditures due to higher inpatient and ED utilization. ${ }^{14}$ In the only study using nationally representative data, Banerjee and colleagues (2010) examined the Medical Expenditure Panel Survey (MEPS) 2000-2004 to study the impact of Medicaid instability on health care utilization, and found that individuals with discontinuous Medicaid coverage had higher inpatient and ED utilization, but lower prescription drug utilization annually. ${ }^{15}$

The literature examining the impact of lack of insurance on health care outcomes in general, and disease-specific outcomes in particular is exhaustive with studies highlighting the adverse impact of lacking health insurance. The literature examining the impact of gaps in health insurance is less comprehensive, however, for the most part this literature has established that even short gaps in insurance coverage may lead to negative outcomes among individuals with chronic conditions. Although these studies have assessed the impact of insurance gaps on patients with numerous conditions including cancer, depression/anxiety and schizophrenia, and diabetes, fewer studies have focused on patient with cardiovascular disease (CVD) and CVD-associated conditions. Studies using more advanced statistical analyses such as zero-inflated linear models, differencein-differences analyses, and instrumental variable regression analyses need to be conducted more to account for endogeneity problems that may confound the association between continuation and discontinuation in Medicaid and other insurance programs and major clinical outcomes. Studies examining Medicaid instability have mostly been conducted using data on a single state's Medicaid program, and there is a paucity of studies (could also be due to lack of national data) that examine nationally representative estimates of churning in Medicaid and its impact on health care outcomes, especially among individuals diagnosed with chronic disease conditions. Although medication adherence/compliance as an area of research has been thoroughly examined with several high-quality robust analyses of adherence, and its impact of health care utilization, research examining the impact of churning in insurance in general and Medicaid in particular is lacking. It is imperative therefore, to examine the impact of Medicaid discontinuity on pharmacotherapy outcomes, such as medication adherence and medication utilization. 


\section{CARDIOVASCULAR DISEASE OR ASSOCIATED HIGH-RISK CONDITIONS AMONG LOW-INCOME POPULATIONS}

CVD is the leading cause of death in the U.S. accounting for approximately 1 in every 4 deaths being attributed to them. ${ }^{16}$ Cardiovascular complications resulting from high blood pressure, lipid disorders, and other risk factors have been known to exert a substantial burden leading to disability, morbidity, and mortality. ${ }^{17,18}$ The economic burden due to CVD has been estimated to exceed $\$ 300$ billion. ${ }^{19}$ Estimates peg the prevalence of cardiovascular disease (CVD), including heart failure and stroke, among low-income adult population at $28 \% .{ }^{20}$ Additionally, many individuals with CVD that have resulted from worsening of other chronic conditions such as hypertension, diabetes, and hyperlipidemia. High blood pressure (24\%), high cholesterol (17\%), and high blood sugar level $(9 \%)$ are some of the prevalent risk factors or high-risk conditions for CVD. ${ }^{21,22}$ For instance, $82 \%$ of low-income diabetic adults, and 74\% of low-income adults with CVD reported one or more additional chronic condition, which indicates the complex disease burden and health care needs in the CVD or high risk population. Additionally, spending for nonelderly adult Medicaid enrollees with CVD and many of the high-risk conditions for CVD ranged from $\$ 9,694$ - $\$ 13,490$ per capita, which was significantly higher than their counterparts without these chronic conditions, and once again reflected the complex chronic disease burden among these diseased subpopulations. ${ }^{20}$

Low-income populations with CVD or conditions placing them at high risk for CVD, such as acute myocardial infarction, congestive heart failure, coronary artery disease, peripheral and visceral atherosclerosis, stroke, hypertension, diabetes, hyperlipidemia, and chronic kidney disease, would face tremendous challenges in managing these complex chronic conditions. CVD and CVD-associated conditions require appropriate and uninhibited access to medical care, preventive care, prescription medications, and periodic primary and specialty care visits for prevention and treatment. Failure to appropriately manage these chronic conditions may exacerbate the symptoms and lead to worsening of health leading, resulting in unplanned hospitalizations and emergency room admissions. Insurance coverage is a critical component and an important factor in ensuring access to and utilization of needed medical care for appropriate disease management. Medicaid, as mentioned above is a critical safety-net health insurance program for low-income populations that provides these vulnerable populations the ability to seek needed medical care. Interruptions and discontinuity in Medicaid coverage may especially have a lead to significantly poor health outcomes among these vulnerable populations, and therefore policies to stabilize Medicaid coverage among low-income populations with CVD or high-risk conditions for CVD may be even more critical to ensuring continuity of care and appropriate disease management among these individuals. While need appropriate medical care, pharmacotherapy is also an important component of disease management for these individuals. Prescribed medicines not only need to be filled in a timely manner as directed, but adhering to the appropriate prescribed medication regimen is also critical to observe positive therapeutic effects of these medications. After reviewing the literature for the definitions for CVD and conditions which are high risk factors, and in 
consultation with a cardiologist to restrict the number of these conditions to those requiring appropriate and prolonged disease management in the outpatient and community settings, the present study has considered the CVD and high-risk conditions for CVD listed above as the index chronic conditions. ${ }^{17,23-26}$ The rationale being that continuity of Medicaid coverage may be even more critical among this subpopulation of the non-elderly adult Medicaid population, and the adverse outcomes due to Medicaid discontinuity may be more detrimental among these individuals.

\section{RESEARCH AIMS}

Using nationally representative data on non-institutionalized, non-elderly adults diagnosed with one or more CVD or high-risk conditions for CVD who reported having Medicaid at any point of time during the year, this dissertation aims to investigate the associations between instability in Medicaid coverage within a year, and access to care, preventive care, prescription drug adherence and utilization, primary care visits, and inpatient and ER hospitalizations.

\section{Aim 1: To Examine the Individual Characteristics Associated with Medicaid Discontinuity}

1) Are the demographic, socioeconomic, Medicaid-, and health-related characteristics significantly different between those who have continuous Medicaid coverage and their counterparts with discontinuous Medicaid coverage during the year?

2) Which characteristics are significantly associated with Medicaid discontinuity in this study population?

\section{Aim 2: To Examine the Associations between Medicaid Discontinuity, Medication Adherence and Medication Utilization}

- Hypothesis 2a: Medicaid discontinuity is associated with poor adherence to commonly prescribed therapeutic classes of medications used to treat CVD or high-risk conditions for CVD

- Hypothesis 2b: Medicaid discontinuity is associated with lower all-cause and disease-specific prescription drug utilization

\section{Aim 3: To Examine the Associations between Medicaid Discontinuity, Access to Care, Preventive Services Use, and Utilization of Health Care Services}

- Hypothesis 3a: Medicaid discontinuity is associated with poor access to care

- Hypothesis 3b: Medicaid discontinuity is associated with a lower likelihood of adequate preventive services use 
- Hypothesis 3c: Medicaid discontinuity is associated with lower all-cause and disease-specific hospital outpatient visits, and office-based physician visits

- Hypothesis 3d: Medicaid discontinuity is associated with higher all-cause and disease-specific inpatient hospitalizations and ER hospitalizations 


\section{CHAPTER 2. CHARACTERISTICS ASSOCIATED WITH MEDICAID DISCONTINUITY IN ADULTS WITH CARDIOVASCULAR DISEASE OR ASSOCIATED HIGH-RISK CONDITIONS}

\section{BACKGROUND}

Cardiovascular disease (CVD) and CVD associated conditions (e.g. hypertension, lipid disorders, and diabetes) exert substantial burden due to disability, morbidity, and mortality. CVD is the major cause of death in the U.S., accounting for 1 in 4 deaths being attributed to them. ${ }^{16}$ Economic burden due to these debilitating chronic conditions is estimated to exceed $\$ 300$ billion annually. ${ }^{19}$ Continuity of care is essential to for appropriate management of these disease conditions, and having uninterrupted access to health insurance is critical to ensure this continuity of care. ${ }^{27,28}$ Medicaid, the nation's primary public health insurance program for the low-income population, provides muchneeded health insurance coverage to an estimated 67 million individuals at any given point in time. ${ }^{7}$ Estimates indicate that over $28 \%$ of the Medicaid population at or below 138 percent of the federal poverty level (FPL) are diagnosed with CVD or high-risk conditions for CVD. ${ }^{29,30}$

Medicaid however, has been described as a leaky sieve. ${ }^{8}$ Inefficient administrative hurdles and ponderous periodic paperwork requirements, result in discontinuity in Medicaid coverage, characterized by wither loss of coverage or "churning" - frequent transitions into and out of various sources of coverage. ${ }^{8,9}$ Such instability often plagues Medicaid-eligible individuals, leading to both gaps in coverage and interruptions in the continuity of care. Families may have to find new providers or change their existing health treatments due to different provider networks or different services covered in their new health plans, or may end up forgoing needed care after becoming uninsured.

The consequences of lack of insurance have been well-documented. Ayanian and colleagues reported that Americans who were uninsured for one year or more were more likely to have foregone routine clinical checkup in the past 2 years, and this pattern was observed among individuals diagnosed with several chronic conditions such as hypertension, hyperlipidemia, HIV/AIDS, among others. Long-term and short-term uninsured (uninsured for less than one year), also did not receive recommended cancer screening, and diabetes care among others. ${ }^{31}$ Recent estimates indicate that these trends have continued into the first decade of the $21^{\text {st }}$ century. Fields and colleagues analyzed the Medical Expenditure Panel Survey (MEPS) data from 2006-2010 to examine the impact of insurance stability and residence on several health care utilization measures. They found that discontinuously insured individuals had more ED visits, fewer physician office visits, and fewer prescription drug fills among others, compared to those with continuous insurance coverage. ${ }^{32}$ Several other studies examining Medicaid data have also reported adverse impacts on access to care, and health outcomes due to gaps in Medicaid coverage. ${ }^{2,10-14}$ 
Medicaid discontinuity, due to either becoming ineligible or "drop-out" despite meeting the eligibility criteria, has been well documented in children. ${ }^{33-35}$ Some research has been conducted to examine Medicaid instability among adults, although this area of research is not exhaustive. For instance, estimates peg the rate of adult disenrollment from Medicaid to range from $21-33 \%$ annually. ${ }^{36-38}$ Sommers examined the predictors of loss of Medicaid and found that young age, male gender, and Hispanic ethnicity were significant risk factors for coverage discontinuity. ${ }^{38}$ However, there have been no estimates of Medicaid instability among non-elderly adults with CVD or high-risk conditions for CVD among whom, the impact of such coverage discontinuity could be detrimental. Including population groups diagnosed with and managing these chronic conditions in policy discussions to improve Medicaid retention among adults is important, as these groups could benefit the most from coverage stability and the resulting continuity in disease management.

The present study aims to: 1) obtain national estimates of Medicaid coverage transitions and discontinuity among non-elderly adults with CVD or high-risk conditions for CVD, 2) characterize the population groups that have continuous and discontinuous Medicaid coverage within a year, and 3) examine the individual characteristics associated with Medicaid discontinuity in this study population.

\section{METHODS}

The present study is a retrospective cross-sectional analysis designed to compare the characteristics of non-elderly adults diagnosed with CVD or high-risk conditions for CVD with continuous full-year Medicaid coverage versus those with discontinuous Medicaid coverage. The study seeks to determine the individual characteristics associated with Medicaid discontinuity in this CVD population. Medicaid, being a means tested source of public health insurance, is not a permanent source of insurance coverage. As discussed earlier, the requirements of periodic reporting of income, assets, family status and residence, apart from changes in one or more of these circumstances are the major sources of instability in Medicaid coverage which may impact access to care and preventive services use for individuals with CVD or high-risk conditions for CVD. In order to capture this instability in the study population, we first describe the prevalence of these chronic conditions in this study population. We then examine in detail, the demographic, socioeconomic, Medicaid-, and health-related characteristics associated with Medicaid discontinuity in this study, and control for these covariates in the multivariate models to determine the individuals characteristics associated with Medicaid discontinuity in this CVD population.

\section{Data Source}

This study analyzed the Medical Expenditure Panel Survey (MEPS). MEPS is a set of large-scale surveys of families, individuals, their medical providers and employers across the United States. It is jointly sponsored by the Agency for Healthcare Research 
and Quality (AHRQ) and the National Center for Health Statistics (NCHS), and has been conducted annually since 1996. It has two major components; the Household Component (HC), and the Insurance Component, with data on medical providers in the HC being supplemented by the Medical Provider Component. MEPS collects detailed information from a nationally representative sample of the civilian noninstitutionalized population of the U.S. on health services utilization and health expenditures, insurance coverage, and sources of payment. ${ }^{39}$

When obtaining national estimates from surveys such as MEPS, appropriate sample size is critical to obtain reliable estimates, since estimates of some population subgroups may vary from year to year. AHRQ suggests a minimum of 100 unweighted participants per cell for producing reliable national estimates. The advantage of survey such as MEPS is that they allow for several years' of data to be pooled together in order to increase the sample size and improve the precision of estimates. The pooling of the different years of data was conducted in accordance with the guidelines provided by MEPS, and data for the years $2002-2011$ were pooled together to allow for a sufficiently large sample size to ensure reliable estimates.

\section{MEPS Sampling Plan}

MEPS has an overlapping panel design. Two separate panels of respondents are interviewed simultaneously during a calendar year. A new panel of sample households is selected each year, and data for each panel are collected for two calendar years. The two years of data for each panel are collected in five rounds of interviews that take place over a two and a half year period. This provides continuous and current estimates of health care expenditures at both the person and household level for two panels for each calendar year. Figure 2-1 illustrates this overlapping panel design in detail with an example of Panels 15 and 16.

\section{Study Population}

The study population consisted of individuals diagnosed with CVD or high-risk conditions for CVD. CVD was defined as any diagnosis of acute myocardial infarction (Clinical Classification code (CCC): 100), coronary artery disease (CCC: 101), congestive heart failure (CCC: 108), peripheral and visceral atherosclerosis (CCCs: 114, 115, 116), stroke (CCCs: 109, 112). High-risk conditions for CVD were defined as hypertension (CCC: 098), lipid disorders (CCC: 053), diabetes (CCC: 049, 050), and chronic kidney disease (CCC: 158). CCC aggregates conditions and procedures into mutually exclusive and clinically homogeneous categories, using Clinical Classification Software. ${ }^{40}$ The study population included respondents who were: (1) aged 18 to 64 years, (2) self-reported either being diagnosed with CVD or high-risk conditions for CVD in their first round of the MEPS survey, or reported having an event (an inpatient, emergency, outpatient, or office-based provider visits, or a prescription medication) associated with one or more of the aforementioned disease conditions at any point of time 


\begin{tabular}{|c|c|c|c|c|c|c|c|c|c|c|c|c|}
\hline & \multicolumn{4}{|c|}{2010} & \multicolumn{4}{|c|}{2011} & \multicolumn{4}{|c|}{2012} \\
\hline & Q1 & Q2 & Q3 & Q4 & Q1 & Q2 & Q3 & Q4 & Q1 & Q2 & Q3 & Q4 \\
\hline \multicolumn{13}{|l|}{$\begin{array}{c}\text { Panel } 14 \\
\text { Round } 3 \\
\text { Round } 4 \\
\text { Round } 5\end{array}$} \\
\hline \multicolumn{13}{|l|}{$\begin{array}{l}\text { Panel } 15 \\
\text { Round } 1 \\
\text { Round } 2 \\
\text { Round } 3 \\
\text { Round } 4 \\
\text { Round } 5\end{array}$} \\
\hline $\begin{array}{l}\text { Panel } 16 \\
\text { Round } 1 \\
\text { Round } 2 \\
\text { Round } 3 \\
\text { Round } 4 \\
\text { Round } 5\end{array}$ & & & & & & & & & & & & \\
\hline $\begin{array}{c}\text { Panel } 17 \\
\text { Round } 1 \\
\text { Round } 2 \\
\text { Round } 3\end{array}$ & & & & & & & & & & & & \\
\hline Sample Size & \multicolumn{4}{|c|}{$N=31,228$} & \multicolumn{4}{|c|}{$N=33,622$} & \multicolumn{4}{|c|}{$\mathrm{N}=37,182$} \\
\hline
\end{tabular}

N is equal to the number of people with a positive person weight on the file.

\section{Figure 2-1. MEPS Overlapping Panel Design}

Reprinted with permission. MEPS-HC Sample Design and Collection Process. Agency for Healthcare Research and Quality, Rockville, Md.

http://www.meps.ahrq.gov/survey_comp/hc_data_collection.jsp. Accessed on April 6, $2015 .^{41}$ 
during the survey, (3) reported having had Medicaid coverage at any point of time during the survey year, and (4) had positive person weights, and were interviewed for all five rounds of the 2-year MEPS survey interviews. Events associated with any particular disease conditions were self-reported and coded by the MEPS data reviewers accordingly. A portion of the self-reported data were verified by contacting the health care providers associated with the events reported and coded accordingly. ${ }^{42}$ Selection of individuals in the study population on the basis of a medical event associated with the disease condition of interest is a valid approach to ensure that the study sample is not impacted by misreporting of chronic conditions diagnoses. ${ }^{43}$ Respondents who reported having Medicare, or pregnancy were excluded from this study.

A major goal of this study was to characterize Medicaid discontinuity among individuals with CVD or high-risk conditions for CVD. Unlike previous studies, this study accounted for presence of other sources of health coverage among the discontinuous Medicaid coverage groups. ${ }^{15}$ This was done since individuals who do not report having continuous full-year Medicaid coverage and have no other health insurance during the year may be different from those with other sources of health insurance coverage, in terms of characteristics and health care utilization. Individuals in the study population who reported having Medicaid coverage during all 12 months of a survey year were classified as having continuous Medicaid coverage, which was the comparison group and designated as 'Continuous Medicaid' in this study. Individuals who reported having $<12$ months of Medicaid coverage during a survey year were divided into two groups: (1) individuals with $<12$ months of Medicaid coverage who did not report any other sources of health insurance during the survey year, designated in this study as 'Discontinuous-Uninsured', and (2) individuals with $<12$ months of Medicaid coverage who also reported having other source(s) of health insurance during the survey year (either private, Tricare, or any hospital- or physician group-based health insurance), designated in this study as 'Discontinuous-Insured'.

\section{Study Variables}

Our primary outcomes of interest are an indicator for Medicaid discontinuity without other health insurance coverage (Discontinuous-Uninsured), and an indicator for Medicaid discontinuity with other sources of health insurance coverage (DiscontinuousInsured). The measures of comparison between the three groups, which were also adjusted for in the regression models included the following covariates:

1) Demographic covariates: age (in years), gender (female vs. male), race/ethnicity (Non-Hispanic White vs. Non-Hispanic Black, Hispanic, and other races), marital status (not married vs. married), family size (N), and geographic residence (Northeast vs. Midwest, South, and West).

2) Socioeconomic covariates: education (less than high school vs. high school/GED, and Bachelor's or higher), income as a percentage of the federal poverty level (FPL) $(<100 \%$ FPL vs. $100-<125 \%$ FPL, and $\geq 125 \%$ FPL), employment (always unemployed vs. intermittent employment, and always employed), binary indicator 
for Metropolitan Statistical Area (MSA) residence.

3) Medicaid-related covariates: binary indicator for Supplementary Security Income (SSI) for disability receipt or participation in the Temporary Assistance for Needy Families (TANF) program, and a binary indicator for Medicaid HMO/Managed Care participation.

4) Health-related covariates: perceived health status (excellent/very good/good vs. fair/poor), perceived mental health status (excellent/very good/good vs. fair/poor), number of CVD or high-risk conditions for CVD $(\mathrm{N})$, binary indicator for mental illness/substance abuse diagnosis, binary indicator for respiratory diseases diagnosis, binary indicator for arthritis/joint pain diagnosis, and Charlson Comorbidity Index (CCI) score.

All regression models included the year fixed effects to capture any macro-level variations during the study period.

\section{Statistical Analysis}

Survey-weighted proportions of prevalence of CVD or high-risk conditions for CVD were calculated. Chi-square tests and t-tests were conducted as part of the initial bivariate statistics to compare characteristics across the groups in the study population. Survey-weighted multivariate logistic regression analyses were then conducted to determine the predictors of Medicaid discontinuity in the study population. As denoted in Equation 2-1 and Equation 2-2,

$$
\begin{aligned}
& \operatorname{Pr}[\mathrm{DU}=1 \mid \mathrm{x}]=\mathrm{F}^{-1}\left(\hat{\alpha}+\sum_{k=1}^{K} \hat{\beta}_{k} X_{k}\right) \\
& \operatorname{Pr}[\mathrm{DI}=1 \mid \mathrm{x}]=\mathrm{F}^{-1}\left(\hat{\alpha}+\sum_{k=1}^{K} \hat{\beta}_{k} X_{k}\right)
\end{aligned}
$$

In the logistic regression analyses, both Discontinuous-Uninsured (denoted by DU) and Discontinuous-Insured (denoted by DI) were modeled as dichotomous outcome variables as a function of the demographic, socioeconomic, Medicaid eligibility and health-related covariates. $\mathrm{F}^{-1}$ denotes the inverse of the cumulative standard logistic distribution function that relates the outcome on the probability scale to the covariates. $\operatorname{Pr}[$ ] denotes the population average probability of Medicaid discontinuity (for both with and without other sources of insurance coverage models) conditional on the covariates. $X$ denotes the vector of aforementioned individual characteristics that predict Medicaid discontinuity in the two population groups of interest. The proportion of missing data was approximately $6 \%$ for the study. As a result, the listwise deletion approach was used to account for missing values. Multicollinearity was assessed for all the regression models, and the variance inflation factor was found to be less than 5, which was lower than the widely accepted threshold of 10 for existence of multicollinearity. The HosmerLemeshow tests for goodness-of-fit were highly insignificant for all the logistic regression models indicating no significant difference between the observed and predicted values of the response variables, and that the model fit the data. All data 
analyses were conducted using SAS 9.3 and STATA 13 to account for the complex survey design of MEPS.

\section{RESULTS}

Figure 2-2 is a schematic representation of the process employed for deriving the unweighted, unadjusted study sample. Of the 344,933 individuals in the ten-year period between 2002 and 2011, 328,135 had positive MEPS person-level weights. After excluding individuals with Medicare (44,921), those who reported pregnancy (17), and those respondents who were not in-scope throughout the survey period $(7,359), 275,838$ non-institutionalized study respondents remained. Of these, 231,675 respondents who did not report being diagnosed with CVD or high-risk conditions for CVD were excluded, resulting in a total of 41,163 individuals being selected who either reported being diagnosed with, or having an event (prescribed medicine, inpatient, ER, outpatient, or office-based provider visits) associated with CVD or high-risk conditions for CVD, which were the index chronic conditions considered in this study. Of these, a total of 38,186 individuals were further excluded as they either did not have Medicaid coverage at any point of time during the study period, or they were less than 18 or more than 64 years of age, resulting in a total overall sample of 5,977 individuals aged 18-64 years who had Medicaid at any given point in time during the study period.

The overall study sample $(5,977)$ was divided further into groups: the continuous Medicaid coverage group consisting of 3,926 individuals (65.7\%) who had uninterrupted Medicaid coverage throughout the year (hereby referred to as Continuous Medicaid), and the discontinuous Medicaid coverage group consisting of 1,900 individuals $(31.8 \%)$ who had less than 12 months of Medicaid coverage. There were 151 individuals $(2.5 \%)$ who reported both full-year Medicaid coverage along with other sources of health insurance, which were excluded in the main analyses and included in the sensitivity analyses. In order to capture the heterogeneity in the discontinuous Medicaid subpopulation due to presence of other sources of health insurance coverage, this group was further divided into two groups: the Discontinuous-Uninsured, which consisted of 1,407 individuals (74.1\% of the discontinuous Medicaid subpopulation, $23.5 \%$ of the total study sample) who did not report any other form of health insurance during the year, and the Discontinuous-Insured, consisting of 493 individuals $(25.9 \%$ of the discontinuous Medicaid subpopulation, $8.3 \%$ of the total study sample) who reported some other form of health insurance during the year (either, private, Tricare, or other forms of hospital- or physician group-based health insurance). Thus, all outcome models were analyzed separately; comparing Continuous Medicaid and the Discontinuous-Uninsured populations, and separately comparing the Continuous Medicaid vs. the DiscontinuousInsured population groups. This was done to capture the variability among the population group of interest, those with Discontinuous Medicaid. Individuals who do not report having continuous full-year Medicaid coverage and have no other health insurance during the year may have significant differences, not only in sociodemographic characteristics and health status, but may also have different health care utilization patterns and health behaviors. 


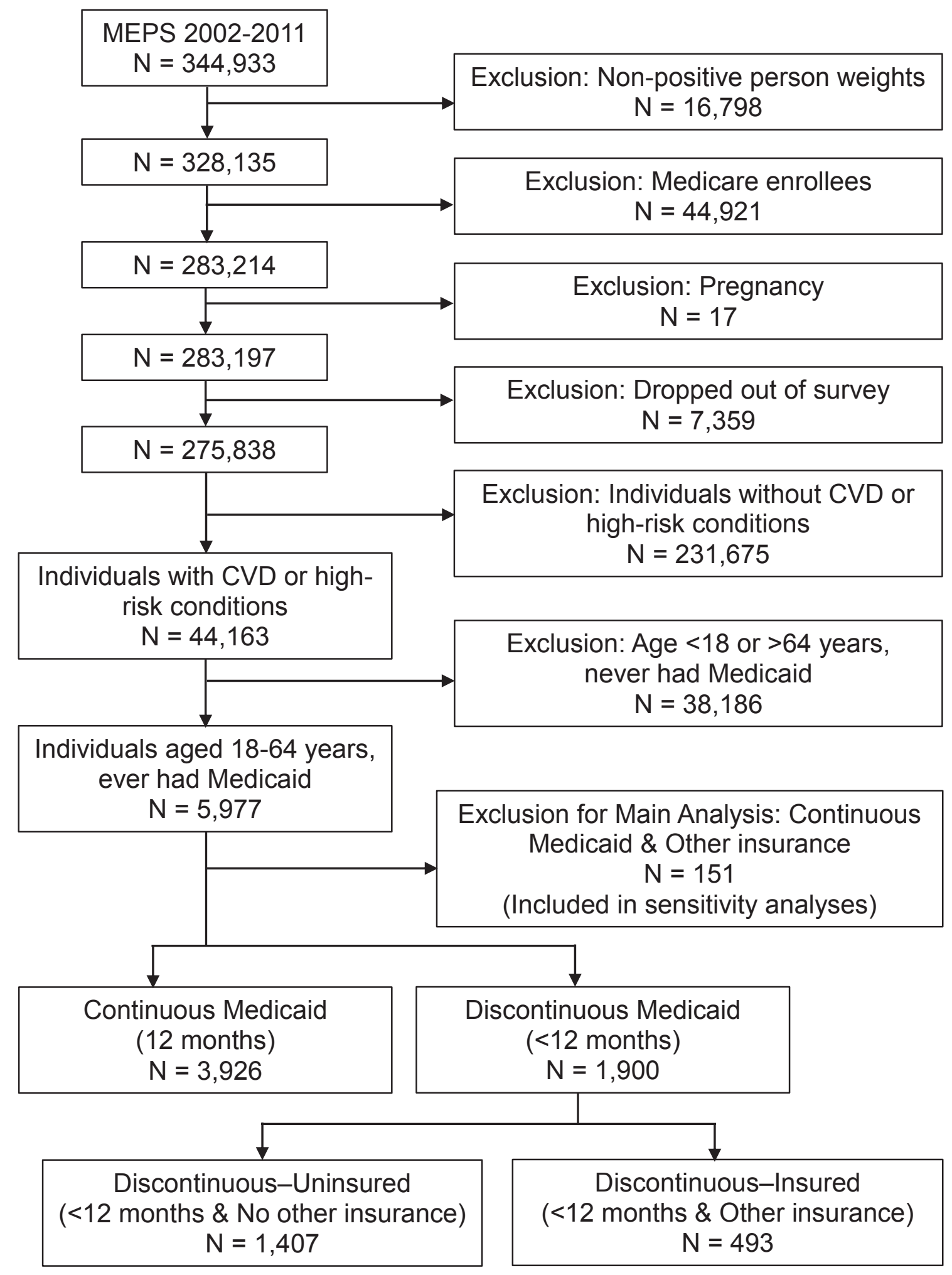

Figure 2-2. Schematic Representation of the Study Sample Derivation Process 
Table 2-1 depicts the distribution of the various diagnosis and utilization components or criteria which were considered in this study for sample selection. From an overall sample of 5,997, 3,997 reported being diagnosed with one or more of the CVD or high-risk conditions for CVD examined in this study, 5,332 reported taking on or more prescription medicines associated with these index chronic conditions, and 3,713 had reported having one or more office-based provider visits for one or more of the index chronic conditions examined in this study.

\section{Prevalence of CVD or High-Risk Conditions for CVD}

Table 2-2 shows the prevalence of CVD or high-risk conditions for CVD in the study population. The prevalence of the index disease conditions was fairly even between the Continuous and Discontinuous-Uninsured groups with hypertension being the most prevalent disease ( $49.9 \%$ vs. $52.7 \%, P=0.179)$, followed by lipid disorders $(34.1 \% \mathrm{vs}$. $31.3 \%, P=0.139)$, and diabetes $(28.3 \%$ vs. $28.5 \%, P=0.918)$. The prevalence of coronary artery disease $(8.1 \%$ vs. $7.3 \%, P=0.407)$, MI ( $4.3 \%$ vs. $4.0 \%, P=0.739)$, stroke $(4.4 \%$ vs. $3.8 \%, P=0.572)$, $\mathrm{CHF}(3.3 \%$ vs. 3.1\%, $P=0.856)$, peripheral and visceral atherosclerosis $(1.6 \%$ vs. $0.9 \%, P=0.082)$, and chronic kidney disease $(0.8 \%$ vs. $0.4 \%, P=0.293)$ had lower prevalence in these groups. Distribution of the index conditions when comparing the Continuous with the Discontinuous-Insured groups was similar. The mean number of index chronic conditions was higher among the Continuous Medicaid group compared to the Discontinuous-Uninsured $(P=0.028)$, and the Discontinuous-Insured groups $(P=0.008)$. When comparing the raw numbers of cardiovascular disease and associated comorbidities in this study population, this study found that $18.7 \%$ of individuals in the Continuous group had 3 or more chronic conditions, compared to $17.7 \%$ in the Discontinuous-Uninsured $(P=0.792)$, and $13.6 \%$ in the Discontinuous-Insured groups $(P=0.096)$.

Among individuals with MI, the most prevalent chronic comorbid index driving diagnosis was hypertension, followed by lipid disorders, coronary artery disease, diabetes, stroke, and congestive heart failure. In this subgroup, the prevalence of stroke was significantly different between the Continuous and Discontinuous-Insured groups $(P=0.027)$. When examining individuals with $\mathrm{CHF}$, the most prevalent chronic comorbid index driving diagnosis was found to be hypertension, followed by lipid disorders, diabetes, coronary artery disease, myocardial infarction, and stroke. Finally, among individuals with coronary artery disease, hypertension was again the most prevalent comorbidity, followed by lipid disorders, diabetes, myocardial infarction, and congestive heart failure (results not shown).

\section{Medicaid Enrollment among the Two Discontinuous Medicaid Groups}

Table 2-3 shows the summary statistics for Medicaid enrollment among the discontinuous Medicaid insurance groups. The average duration of enrollment for the undivided discontinuous Medicaid population was 6.0 months. The Discontinuous- 
Table 2-1. Diagnosis and Event Components Used to Select the Study Population

\begin{tabular}{lccc}
\hline \multicolumn{1}{c}{ Components } & Frequency (N) & $\begin{array}{c}\text { Weighted } \\
\text { Frequency (N) }\end{array}$ & \% (SE) \\
\hline $\begin{array}{l}\text { CVD or High-Risk Conditions } \\
\text { for CVD }\end{array}$ & 3,997 & $2,638,024$ & $67.8(0.9)$ \\
$\begin{array}{l}\text { Rx for CVD or High-Risk } \\
\text { Conditions for CVD }\end{array}$ & 5,332 & $3,474,117$ & $89.3(0.6)$ \\
$\begin{array}{l}\text { Inpatient Visit for CVD or High- } \\
\text { Risk Conditions for CVD }\end{array}$ & 372 & 260,990 & $6.7(0.4)$ \\
$\begin{array}{l}\text { ER Visit for CVD or High-Risk } \\
\text { Conditions for CVD }\end{array}$ & 463 & 304,458 & $7.8(0.5)$ \\
$\begin{array}{l}\text { Outpatient Visit for CVD or } \\
\text { High-Risk Conditions for CVD }\end{array}$ & 480 & 324,800 & $8.3(0.6)$ \\
$\begin{array}{l}\text { Office-Based Visit for CVD or } \\
\text { High-Risk Conditions for CVD }\end{array}$ & 3,713 & $2,379,045$ & $61.1(0.9)$ \\
\begin{tabular}{l} 
Study Population \\
\hline
\end{tabular} & 5,977 & & \\
\hline
\end{tabular}

CVD: Cardiovascular disease; Rx: Prescription medication; ER: Emergency room. CVD include myocardial infarction, coronary artery disease, congestive heart failure, peripheral and visceral atherosclerosis, stroke. High-risk conditions for CVD include hypertension, lipid disorders, and diabetes, chronic kidney disease.

Study Population includes individuals reporting one or more of at least one of the study components listed in the table. 
Table 2-2. Prevalence of CVD or High-Risk Conditions for CVD in the Study Population

\begin{tabular}{|c|c|c|c|c|c|c|c|}
\hline \multirow[b]{2}{*}{ Prevalence } & \multirow[b]{2}{*}{$\begin{array}{c}\text { Index } \\
\text { Diseases } \\
\end{array}$} & \multicolumn{2}{|c|}{ Continuous Medicaid } & \multicolumn{2}{|c|}{ Discontinuous-Uninsured } & \multicolumn{2}{|c|}{ Discontinuous-Insured } \\
\hline & & $\begin{array}{c}\text { Weighted N } \\
\text { or Mean }\end{array}$ & $\begin{array}{r}\%(\mathrm{SE}) \\
\text { or SE } \\
\end{array}$ & $\begin{array}{l}\text { Weighted N } \\
\text { or Mean }\end{array}$ & $\begin{array}{c}\%(\mathrm{SE}) \\
\text { or SE }\end{array}$ & $\begin{array}{c}\text { Weighted N } \\
\text { or Mean }\end{array}$ & $\begin{array}{c}\%(\mathrm{SE}) \\
\text { or SE }\end{array}$ \\
\hline & MI & 109,746 & $4.3(0.5)$ & 36,811 & $4.0(0.6)$ & 10,903 & $2.6(0.9)$ \\
\hline & CAD & 207,594 & $8.1(0.6)$ & 66,361 & $7.3(0.9)$ & 25,846 & $6.1(1.2)$ \\
\hline & $\mathrm{CHF}$ & 83,275 & $3.3(0.5)$ & 28,341 & $3.1(0.8)$ & 5,709 & $1.4(0.7)$ \\
\hline & PVA & 40,962 & $1.6(0.3)$ & 8,511 & $0.9(0.3)$ & 7,810 & $1.8(0.7)$ \\
\hline & Stroke & 111,114 & $4.4(0.5)$ & 35,051 & $3.8(0.8)$ & 13,361 & $3.2(1.1)$ \\
\hline & Hypertension & $1,273,888$ & $49.9(1.2)$ & 481,202 & $52.7(1.8)$ & 196,635 & $46.4(2.7)$ \\
\hline & Lipid Disorders & 870,818 & $34.1(1.1)$ & 285,706 & $31.3(1.7)$ & 133,370 & $31.4(2.5)$ \\
\hline & Diabetes & 723,694 & $28.3(1.1)$ & 260,672 & $28.5(1.8)$ & 113,299 & $26.7(2.2)$ \\
\hline & CKD & 19,144 & $0.8(0.2)$ & 4,046 & $0.4(0.2)$ & 1,323 & $0.3(0.2)$ \\
\hline Mean Index & & 1.73 & 0.01 & 1.70 & $0.01^{*}$ & 1.56 & $0.06^{* *}$ \\
\hline \multicolumn{8}{|l|}{ Diseases (SE) } \\
\hline \multicolumn{8}{|l|}{ No. of Index } \\
\hline \multicolumn{8}{|l|}{ Diseases } \\
\hline 1 & & 951,993 & $54.7(1.4)$ & 351,268 & $56.2(2.2)$ & 170,538 & $62.4(3.2)$ \\
\hline 2 & & 462,928 & $26.6(1.1)$ & 163,062 & $26.1(1.8)$ & 65,581 & $24.0(3.2)$ \\
\hline$\geq 3$ & & 325,033 & $18.7(1.1)$ & 110,621 & $17.7(1.8)$ & 37,000 & $13.6(2.4)$ \\
\hline
\end{tabular}

CVD: Cardiovascular disease; MI: myocardial infarction; CAD: coronary artery disease; CHF: congestive heart failure; PVA: peripheral vascular disease; CKD: chronic kidney disease; SE: standard error

$* * * P<0.001 ; * * P<0.01 ; * P<0.05$ for difference from the Continuous Medicaid group. Index diseases include the CVD or its associated risk factor comorbidities considered in this study. Sample consists of 3,003997 individuals or weighted sample of 2,638,024 who reported being diagnosed with one or more index diseases. 
Table 2-3. Medicaid Enrollment among the Discontinuous Medicaid Groups

\begin{tabular}{|c|c|c|c|c|}
\hline \multirow[b]{2}{*}{$\begin{array}{c}\text { Coverage } \\
\text { Components }\end{array}$} & \multicolumn{2}{|c|}{ Discontinuous-Uninsured } & \multicolumn{2}{|c|}{ Discontinuous-Insured } \\
\hline & $\begin{array}{c}\text { Mean or } \\
\text { Weighted N }\end{array}$ & $\begin{array}{l}\text { SE or \% } \\
\text { (SE) }\end{array}$ & $\begin{array}{c}\text { Mean or } \\
\text { Weighted N }\end{array}$ & $\begin{array}{l}\text { SE or \% } \\
\text { (SE) }\end{array}$ \\
\hline $\begin{array}{l}\text { Mean Duration of } \\
\text { Medicaid }\end{array}$ & 6.5 months & 0.1 & 5.0 months $^{* * *}$ & 0.2 \\
\hline $\begin{array}{l}\text { Mean Duration of } \\
\text { No Insurance }\end{array}$ & 5.5 months & 0.1 & 1.2 months $^{* * *}$ & 0.1 \\
\hline $\begin{array}{l}\text { Insurance Status } \\
\text { After } 12 \text { Months }{ }^{\dagger}\end{array}$ & & & & \\
\hline Still in Medicaid & 83,125 & $21.9(2.2)$ & $11,647^{* * *}$ & $8.0(2.0)$ \\
\hline Uninsured & 297,045 & $78.1(2.2)$ & $10,058^{* * *}$ & $6.0(2.0)$ \\
\hline Other Insurance & -- & -- & 147,896 & $86.0(2.0)$ \\
\hline $\begin{array}{l}\text { Medicaid } \\
\text { Coverage } \\
\text { Transitions }\end{array}$ & & & & \\
\hline 1 & 752,311 & $82.3(1.4)$ & 350,803 & $82.7(2.0)$ \\
\hline$\geq 2$ & 161,615 & $17.7(1.4)$ & 73,462 & $17.3(2.0)$ \\
\hline
\end{tabular}

Sample consisted of 380,171 individuals in the Discontinuous-Uninsured group and 169,600 individuals in the Discontinuous-Insured group who either reported being diagnosed with or having an event (prescription medication, or inpatient, emergency, hospital outpatient, or officebased provider visits) associated with CVD or high-risk conditions for CVD, and who reported having Medicaid at the beginning of the year.

$* * * P<0.001 ; * * P<0.01 ; * P<0.05$ for difference from the Discontinuous-Uninsured group. 
Uninsured group was enrolled in Medicaid for a longer period on average compared to the Discontinuous-Insured group (6.5 vs. 5.0 months, $P<0.001)$. Additionally, this study examined the average duration of no insurance and found individuals in the Discontinuous-Insured group were uninsured for a little over a month (5.5 vs. 1.2 months, $P<0.001$ ). This study also examined Medicaid enrollment status after 12 months of initial Medicaid enrollment. Overall, a total of 4,898 individuals were enrolled in the month of January of the survey year, of which $85.3 \%$ were still enrolled in Medicaid during the month of December in the survey year, $3.5 \%$ had some other form of insurance coverage, and $10.2 \%$ were uninsured (results not shown). Of the 621 individuals in the Discontinuous-Uninsured group who had Medicaid in the beginning of the year, $78.1 \%$ were uninsured while $21.9 \%$ still had Medicaid coverage at the end of the year. Of the 200 individuals in the Discontinuous-Insured group who had Medicaid in the beginning of the survey year, only $8.0 \%$ still had Medicaid, $6.0 \%$ were uninsured, and the remaining $86 \%$ had some other source of health insurance coverage at the end of the year. Finally, when examining the number of transitions into and/or out of Medicaid coverage, this study found that overall $17.6 \%$ of individuals in this study population had more than one transitions, and the proportion of multiple transitions was similar between the two discontinuous Medicaid subgroups ( $17.7 \%$ vs. $17.3 \%, P=0.875)$.

\section{Characteristics of the Study Population}

Table 2-4 displays the demographic, socioeconomic, eligibility, and healthrelated characteristics of the study population. The mean age of the full sample was 46.5 years (not displayed). In comparison to the Continuous Medicaid group, those in the Discontinuous-Uninsured group were less likely to belong to other races $[5.5 \%$ (Discontinuous-Insured) vs. 9.5\% (Continuous), $P<0.001$ ], were more likely to be married ( $37.7 \%$ vs. $29.9 \%, P<0.001)$, and were more likely to belong to the South $(34.5 \%$ vs. $29.8 \%, P=0.035)$. When examining the socioeconomic characteristics, this study found that the Discontinuous-Uninsured were more likely to have completed high school/GED ( $54.9 \%$ vs. $50.4 \%, P=0.004)$ or college $(12.2 \%$ vs. $9.4 \%, P=0.004)$, were more likely to have incomes $\geq 125 \%$ FPL $(43.0 \%$ vs. $33.9 \%, P<0.001)$, and were more likely to have intermittent $(25.0 \%$ vs. $12.4 \%, P<0.001)$, as well as continuous full-year employment $(20.7 \%$ vs. $15.1 \%, P<0.001)$. Among the Medicaid eligibility variables, the Discontinuous-Uninsured were less likely to be eligible for SSI due to disability or participate in TANF $(16.9 \%$ vs. $41.5 \%, P<0.001)$, and were less likely to report Medicaid $\mathrm{HMO} /$ managed care $(50.6 \%$ vs. $61.3 \%, P<0.001)$. Finally, when examining the healthrelated covariates, they were less likely to report having mental /substance abuse illnesses ( $24.9 \%$ vs. $30.8 \%, P=0.003)$, respiratory illnesses $(12.9 \%$ vs. $19.7 \%, P<0.001)$, or arthritis/joint pain diagnosis (29.3\% vs. $34.1 \%, P=0.015)$, and were less likely to report having fair or poor perceived mental health status $(25.7 \%$ vs. $33.7 \%, P<0.001)$ when compared to the Continuous Medicaid group. The mean CCI score was not significantly different between the two groups $(P=0.078)$.

When comparing the Discontinuous-Insured group to the Continuous Medicaid group (Table 2-4), this study found that when examining the demographic covariates, 
Table 2-4. Characteristics of the Study Population

\begin{tabular}{|c|c|c|c|c|c|c|c|c|}
\hline \multirow[b]{2}{*}{ Characteristics } & \multicolumn{2}{|c|}{ Continuous Medicaid } & \multicolumn{3}{|c|}{ Discontinuous-Uninsured } & \multicolumn{3}{|c|}{ Discontinuous-Insured } \\
\hline & Weighted N & $\%(\mathrm{SE})$ & Weighted N & $\%(\mathrm{SE})$ & $P$-value ${ }^{\dagger}$ & Weighted N & $\%(\mathrm{SE})$ & $P$-value ${ }^{\dagger \dagger}$ \\
\hline DEMOGRAPHIC & & & & & & & & \\
\hline Age & & & & & 0.621 & & & 0.027 \\
\hline $18-34$ years & 388,425 & $15.9(1.0)$ & 165,340 & $18.1(1.3)$ & & 98,188 & $23.1(2.3)$ & \\
\hline $35-49$ years & 875,809 & $35.9(1.2)$ & 324,653 & $35.5(1.8)$ & & 141,109 & $33.3(2.7)$ & \\
\hline $50-64$ years & $1,173,144$ & $48.2(1.3)$ & 423,933 & $46.4(2.0)$ & & 184,968 & $43.6(3.0)$ & \\
\hline Gender & & & & & 0.359 & & & 0.223 \\
\hline Female & $1,632,006$ & $67.0(1.2)$ & 624,410 & $68.3(1.8)$ & & 267,502 & $63.1(2.8)$ & \\
\hline Race/Ethnicity & & & & & $<0.001$ & & & $<0.001$ \\
\hline Non-Hispanic White & $1,094,002$ & $44.9(1.8)$ & 442,534 & $48.4(2.1)$ & & 256,231 & $60.4(3.2)$ & \\
\hline Non-Hispanic Black & 666,358 & $27.3(1.5)$ & 219,033 & $24.0(1.6)$ & & 799,36 & $18.8(2.0)$ & \\
\hline Hispanic & 445,601 & $18.3(1.2)$ & 202,106 & $22.1(1.5)$ & & 50,704 & $12.0(1.6)$ & \\
\hline Others & 231,416 & $9.5(0.9)$ & 50,253 & $5.5(0.9)$ & & 37,393 & $8.8(1.7)$ & \\
\hline Marital Status & & & & & $<0.001$ & & & $<0.001$ \\
\hline Married & 727,457 & $29.9(1.3)$ & 344,150 & 37.7 (1.8) & & 184,414 & $43.5(3.2)$ & \\
\hline Region & & & & & 0.035 & & & 0.034 \\
\hline Northeast & 608,070 & $25.0(1.6)$ & 187,785 & $20.5(1.7)$ & & 104,394 & $24.6(2.9)$ & \\
\hline Midwest & 477,401 & $19.6(1.4)$ & 184,710 & $20.2(1.7)$ & & 115,146 & $27.2(3.2)$ & \\
\hline South & 727,520 & $29.8(1.6)$ & 315,073 & $34.5(1.8)$ & & 98,072 & $23.1(2.3)$ & \\
\hline West & 624,386 & $25.6(1.7)$ & 226,358 & 24.8 (1.9) & & 106,652 & $25.1(3.0)$ & \\
\hline
\end{tabular}


Table 2-4. (Continued)

\begin{tabular}{|c|c|c|c|c|c|c|c|c|}
\hline \multirow[b]{2}{*}{ Characteristics } & \multicolumn{2}{|c|}{ Continuous Medicaid } & \multicolumn{3}{|c|}{ Discontinuous-Uninsured } & \multicolumn{3}{|c|}{ Discontinuous-Insured } \\
\hline & $\begin{array}{c}\text { Weighted } \\
\mathbf{N}\end{array}$ & $\%(\mathrm{SE})$ & Weighted $\mathbf{N}$ & $\%(\mathrm{SE})$ & $P$-value ${ }^{\dagger}$ & Weighted N & $\%(\mathrm{SE})$ & $P$-value ${ }^{\dagger \dagger}$ \\
\hline Family Size Mean (SE) & $2.8(0.1)$ & & $2.9(0.1)$ & & 0.481 & $2.8(0.1)$ & & 0.900 \\
\hline \multicolumn{9}{|l|}{ SOCIOECONOMIC } \\
\hline Education & & & & & 0.004 & & & $<0.001$ \\
\hline$<$ High School & 971,041 & $40.2(1.4)$ & 298,300 & $32.9(1.8)$ & & 67,896 & $16.0(1.9)$ & \\
\hline High School/GED & $1,215,968$ & $50.4(1.4)$ & 498,289 & $54.9(2.0)$ & & 232,334 & $54.9(3.2)$ & \\
\hline College Degree & 227,668 & $9.4(0.7)$ & 111,300 & $12.2(1.4)$ & & 123,109 & $29.1(3.2)$ & \\
\hline Income & & & & & $<0.001$ & & & $<0.001$ \\
\hline$<100 \% \mathrm{FPL}$ & $1,384,551$ & $56.8(1.2)$ & 428,540 & $46.9(1.7)$ & & 104,719 & $24.7(2.3)$ & \\
\hline $100-<125 \% \mathrm{FPL}$ & 227,591 & $9.3(0.6)$ & 91,940 & $10.1(1.1)$ & & 28,783 & $6.8(1.3)$ & \\
\hline$\geq 125 \% \mathrm{FPL}$ & 825,236 & $33.9(1.1)$ & 393,445 & $43.0(1.8)$ & & 290,763 & $68.5(2.6)$ & \\
\hline Employment & & & & & $<0.001$ & & & $<0.001$ \\
\hline Always Unemployed & $1,767,234$ & $72.5(1.2)$ & 496,282 & $54.3(1.8)$ & & 112,490 & $26.5(2.2)$ & \\
\hline Unstable Employment & 301,597 & $12.4(0.8)$ & 228,246 & $25.0(1.6)$ & & 129,583 & $30.6(2.5)$ & \\
\hline Always Employed & 368,547 & $15.1(0.9)$ & 189,398 & $20.7(1.4)$ & & 182,191 & $42.9(2.9)$ & \\
\hline MSA & $1,931,797$ & $79.3(1.8)$ & 721,532 & $78.9(1.9)$ & 0.854 & 344,287 & $81.1(2.7)$ & 0.503 \\
\hline \multicolumn{9}{|l|}{ MEDICAID-RELATED } \\
\hline SSI/TANF & $1,012,240$ & $41.5(1.3)$ & 154,157 & $16.9(1.3)$ & $<0.001$ & 41,212 & $9.7(1.6)$ & $<0.001$ \\
\hline Medicaid HMO/MCO & $1,424,726$ & $61.3(1.5)$ & 459,330 & $50.5(2.0)$ & $<0.001$ & 233,429 & $55.0(3.0)$ & 0.064 \\
\hline
\end{tabular}


Table 2-4. (Continued)

\begin{tabular}{|c|c|c|c|c|c|c|c|c|}
\hline \multirow[b]{2}{*}{ Characteristics } & \multicolumn{2}{|c|}{ Continuous Medicaid } & \multicolumn{3}{|c|}{ Discontinuous-Uninsured } & \multicolumn{3}{|c|}{ Discontinuous-Insured } \\
\hline & Weighted N & $\%(\mathrm{SE})$ & Weighted N & $\%(\mathrm{SE})$ & $P$-value ${ }^{\dagger}$ & Weighted N & $\%(\mathrm{SE})$ & $P$-value ${ }^{\dagger \dagger}$ \\
\hline HEALTH-RELATED & & & & & & & & \\
\hline Perceived Health Status & & & & & 0.144 & & & $<0.001$ \\
\hline Fair to Poor & $1,314,794$ & $53.9(1.2)$ & 467,555 & $51.2(1.9)$ & & 170,598 & $40.2(2.5)$ & \\
\hline Perceived Mental Health & & & & & $<0.001$ & & & $<0.001$ \\
\hline Fair to Poor & 821,318 & $33.7(1.2)$ & 234,931 & $25.7(1.7)$ & & 84,716 & $20.0(2.2)$ & \\
\hline $\begin{array}{l}\text { CVD or High-Risk } \\
\text { Conditions for CVD }\end{array}$ & & & & & 0.775 & & & 0.037 \\
\hline$\leq 1$ & $1,678,222$ & $68.9(1.1)$ & 640,242 & $70.1(1.8)$ & & 321,685 & $75.8(2.3)$ & \\
\hline 2 & 444,995 & $18.2(0.8)$ & 163,062 & $17.8(1.4)$ & & 65,581 & $15.5(2.2)$ & \\
\hline$\geq 3$ & 314,160 & $12.9(0.8)$ & 110,621 & $12.1(1.3)$ & & 37,000 & $8.7(1.5)$ & \\
\hline $\begin{array}{l}\text { Mental/Substance Abuse } \\
\text { Illnesses }\end{array}$ & 751,357 & $30.8(1.2)$ & 227,885 & $24.9(1.6)$ & 0.003 & 115,891 & $27.3(2.4)$ & 0.176 \\
\hline Respiratory Illnesses & 479,702 & $19.7(1.1)$ & 117,992 & $12.9(1.1)$ & $<0.001$ & 39,380 & $9.3(1.5)$ & $<0.001$ \\
\hline Arthritis/Joint Pain & 830,408 & $34.1(1.3)$ & 267,358 & $29.3(1.7)$ & 0.015 & 107,575 & $25.4(2.4)$ & 0.002 \\
\hline CCI Score Mean (SE) & $1.2(0.0)$ & & $1.1(0.1)$ & & 0.078 & $0.9(0.1)$ & & 0.003 \\
\hline Population Size & $2,437,378$ & $64.6(1.0)$ & 913,926 & $24.2(0.8)$ & & 424,265 & $11.2(0.7)$ & \\
\hline
\end{tabular}




\section{Table 2-4. (Continued)}

Study population includes MEPS respondents from the years 2002-2011, aged 18-64 years, who either reported being diagnosed with, or reported having an event (prescription medication, or inpatient, emergency, outpatient, or office-based provider visits) associated with, 1 or more cardiovascular disease or associated risk factor comorbidities considered in this study.

${ }^{\dagger} P$-value for the difference between Discontinuous-Uninsured and Continuous Medicaid groups. ${ }^{\dagger} P$-value for the difference between

Discontinuous-Insured and Continuous Medicaid groups. $P$-values in bold indicate statistically significant difference below the threshold value of $P<0.05$.

SE: Standard error; GED: General educational development; FPL: Federal poverty level; MSA: Metropolitan Statistical Area; SSI:

Supplementary Security Income due to disability; TANF: Temporary Assistance to Needy Families program participation; HMO: Health maintenance organization; MCO: Managed care organization; CCI: Charlson Comorbidity Index score (D’Hoore adapted CCI score was calculated in this study using the 3-digit ICD-9-CM codes in MEPS). 
individuals in the Discontinuous-Insured group were more likely to be younger $(23.1 \%$ vs. $15.9 \%$ in the 18 -34 years age group, $P=0.027)$, less likely to be Non-Hispanic Black $(18.8 \%$ vs. $27.3 \%, P<0.001)$ or Hispanic $(12.0 \%$ vs. $18.3 \%, P<0.001)$, more likely to be married (43.5\% vs. $30.4 \%, P<0.001)$, and more likely to reside in the Midwest $(27.2 \%$ vs. $19.6 \%, P=0.034)$. Among the socioeconomic characteristics, the DiscontinuousInsured were more likely to have a college degree $(29.1 \%$ vs. $9.4 \%, P<0.001)$, were more likely to have family incomes $\geq 125 \%$ FPL $(68.5 \%$ vs. $33.9 \%, P<0.001)$, were more likely to have intermittent ( $30.5 \%$ vs. $12.4 \%, P<0.001)$, or continuous full-year employment (42.9\% vs. $15.1 \%, P<0.001)$. Among the Medicaid eligibility variables it was found that the Discontinuous-Insured were less likely to be eligible for SSI due to disability or participate in TANF $(9.7 \%$ vs. $41.5 \%, P<0.001)$, however, Medicaid managed care enrollment was not statistically significant. When examining the health-related characteristics, the Discontinuous-Insured were less likely to report having fair to poor health status $(40.2 \%$ vs. $53.9 \%, P<0.001)$, or fair to poor mental health status $(20.0 \%$ vs. $33.7 \%, P<0.001$ ), more likely to have fewer CVD or high-risk conditions for CVD $(P=0.037)$ less likely to have respiratory illnesses $(9.3 \%$ vs. $19.7 \%, P<0.001)$, or arthritis/joint pain diagnosis ( $25.4 \%$ vs. $34.1 \%, P=0.002)$, and had a lower mean CCI score [Mean (SE): $0.9(0.07)$ vs. $1.2(0.04), P=0.003]$ in comparison to the Continuous Medicaid group.

\section{Characteristics Associated with Medicaid Discontinuity}

The final study sample with non-missing values on all variables, after undergoing the listwise deletion process, was 5,738, comprising of 3,859 individuals in the Continuous Medicaid group, 1,388 individuals in the Discontinuous-Uninsured group, and 491 individuals in the Discontinuous-Insured group. Table 2-5 shows the results of the multivariate logistic regression analyses to determine the predictors of Medicaid discontinuity among non-elderly adults with CVD or high-risk conditions for CVD.

\section{Discontinuous-Uninsured}

When comparing individuals with discontinuous Medicaid coverage with no other sources of health coverage (Discontinuous-Uninsured) to those with continuous Medicaid coverage (Continuous) group, this study found that among the demographic predictors, male respondents had 18\% lower odds than females [Odds Ratio (OR): 0.82; 95\% Confidence Interval (CI): 0.68 - 0.98], Non-Hispanic Blacks had a 21\% lower odds (OR: $0.79,95 \%$ CI: $0.64-0.98$ ), and individuals belonging other races had a $43 \%$ lower odds compared to Non-Hispanic Whites (OR: 0.57, 95\% CI: $0.40-0.81$ ), married individuals had $26 \%$ higher odds than respondents who were not married (OR: 1.26, 95\% CI: 1.03 - 1.55), and individuals living in the South had about $65 \%$ higher odds compared to the Northeast residents (OR: $1.65,95 \%$ CI: $1.29-2.10$ ), of experiencing Medicaid discontinuity with no subsequent (or concurrent) sources of health coverage.

Among the socioeconomic predictors of Medicaid discontinuity, this study found that individuals with family income levels of $\geq 125 \%$ FPL had about $23 \%$ higher odds 
Table 2-5. Characteristics Associated with Medicaid Discontinuity in the Study Population

\begin{tabular}{|c|c|c|}
\hline Characteristics & $\begin{array}{c}\text { Discontinuous-Uninsured } \\
\text { Odds Ratio (95\% Confidence Interval) }\end{array}$ & $\begin{array}{c}\text { Discontinuous-Insured } \\
\text { Odds Ratio (95\% Confidence Interval) }\end{array}$ \\
\hline \multicolumn{3}{|l|}{ DEMOGRAPHIC } \\
\hline \multicolumn{3}{|l|}{ Age } \\
\hline $18-34$ years & 1.00 (Reference) & 1.00 (Reference) \\
\hline $35-49$ years & $1.10(0.83-1.45)$ & $1.12(0.75-1.67)$ \\
\hline $50-64$ years & $1.25(0.92-1.69)$ & $0.85(0.62-1.16)$ \\
\hline \multicolumn{3}{|l|}{ Gender } \\
\hline Female & 1.00 (Reference) & 1.00 (Reference) \\
\hline Male & $0.82(0.68-0.98)^{*}$ & $0.94(0.73-1.21)$ \\
\hline \multicolumn{3}{|l|}{ Race/Ethnicity } \\
\hline Non-Hispanic White & 1.00 (Reference) & 1.00 (Reference) \\
\hline Non-Hispanic Black & $0.79(0.64-0.98)^{*}$ & $0.59(0.43-0.82)^{* *}$ \\
\hline Hispanic & $1.09(0.85-1.38)$ & $0.65(0.44-0.96)^{*}$ \\
\hline Others & $0.57(0.40-0.81)^{* *}$ & $1.01(0.60-1.71)$ \\
\hline \multicolumn{3}{|l|}{ Marital Status } \\
\hline Not Married & 1.00 (Reference) & 1.00 (Reference) \\
\hline Married & $1.26(1.03-1.55)^{*}$ & $1.50(1.09-2.06)^{*}$ \\
\hline \multicolumn{3}{|l|}{ Region } \\
\hline Northeast & $0.86(0.66-1.11)$ & 1.00 (Reference) \\
\hline Midwest & $1.15(0.87-1.52)$ & $1.46(0.97-2.18)$ \\
\hline South & $1.40(1.09-1.80)^{* *}$ & $1.13(0.78-1.63)$ \\
\hline
\end{tabular}


Table 2-5. (Continued)

\begin{tabular}{lcc}
\hline \multicolumn{1}{c}{ Characteristics } & $\begin{array}{c}\text { Discontinuous-Uninsured } \\
\text { Odds Ratio (95\% Confidence Interval) }\end{array}$ & $\begin{array}{c}\text { Discontinuous-Insured } \\
\text { Odds Ratio (95\% Confidence Interval) }\end{array}$ \\
\hline West & 1.00 (Reference) & $0.99(0.67-1.48)$ \\
Family Size & $0.94(0.89-1.00)^{*}$ & $0.86(0.76-0.96)^{*}$ \\
SOCIOECONOMIC & & \\
Education & 1.00 (Reference) & 1.00 (Reference) \\
$<$ High School & $1.22(1.00-1.49)$ & $1.77(1.26-2.49)^{* * *}$ \\
High School/GED & $1.05(0.76-1.45)$ & $3.13(2.16-4.53)^{* * *}$ \\
College Degree & & $1.00($ Reference $)$ \\
Income & $1.00($ Reference) & $1.21(0.77-1.92)$ \\
$<100 \%$ FPL & $1.06(0.80-1.41)$ & $2.52(1.89-3.36)^{* * *}$ \\
$100-<125 \%$ FPL & $1.23(1.01-1.50)^{*}$ & \\
$\geq 125 \%$ FPL & & $1.00($ Reference $)$ \\
Employment & $1.00($ Reference) & $3.97(2.86-5.51)^{* * *}$ \\
Always Unemployed & $1.92(1.49-2.47)^{* * *}$ & $3.52(2.47-5.01)^{* * *}$ \\
Unstable Employment & $1.23(0.96-1.56)$ & $1.00($ Reference $)$ \\
Always Employed & & $1.28(0.85-1.92)$ \\
MSA & $1.00($ Reference) & \\
Non-MSA & $1.08(0.85-1.37)$ & \\
MSA & &
\end{tabular}


Table 2-5. (Continued)

\begin{tabular}{lll}
\hline \multicolumn{1}{c}{ Characteristics } & $\begin{array}{c}\text { Discontinuous-Uninsured } \\
\text { Odds Ratio (95\% Confidence Interval) }\end{array}$ & $\begin{array}{c}\text { Discontinuous-Insured } \\
\text { Odds Ratio (95\% Confidence Interval) }\end{array}$ \\
\hline MEDICAID-RELATED & & 1.00 (Reference) \\
SSI/TANF & 1.00 (Reference) & $0.33(0.21-0.51)^{* * *}$ \\
No & $0.33(0.27-0.41)^{* * *}$ & 1.00 (Reference) \\
Yes & & $0.70(0.53-0.92)^{*}$ \\
Medicaid HMO/MCO & 1.00 (Reference) & \\
No & $0.61(0.52-0.73)^{* * *}$ & 1.00 (Reference) \\
Yes & & $1.09(0.83-1.42)$ \\
HEALTH-RELATED & & \\
Perceived Health Status & 1.00 (Reference) & 1.00 (Reference) \\
Excellent/Very Good/Good & $1.25(1.04-1.51)^{*}$ & $0.84(0.60-1.17)$ \\
Fair to Poor & & \\
Perceived Mental Health & $1.00($ Reference) & \\
Excellent/Very Good/Good & $0.83(0.67-1.02)$ & 1.00 (Reference) \\
Fair to Poor & & $0.80(0.54-1.17)$ \\
CVD or High-Risk Conditions for & 1.00 (Reference) & $0.84(0.50-1.41)$ \\
CVD & $1.05(0.84-1.32)$ & \\
$\leq 1$ & $0.97(0.69-1.36)$ & \\
2 & &
\end{tabular}


Table 2-5. (Continued)

\begin{tabular}{lcc}
\hline \multicolumn{1}{c}{ Characteristics } & $\begin{array}{c}\text { Discontinuous-Uninsured } \\
\text { Odds Ratio (95\% Confidence Interval) }\end{array}$ & $\begin{array}{c}\text { Discontinuous-Insured } \\
\text { Odds Ratio (95\% Confidence Interval) }\end{array}$ \\
\hline Mental/Substance Abuse Illnesses & 1.00 (Reference) & 1.00 (Reference) \\
No & $0.91(0.73-1.15)$ & $1.23(0.90-2.18)$ \\
Yes & & \\
Respiratory Illnesses & 1.00 (Reference) & $1.00($ Reference) \\
No & $0.72(0.56-0.91)^{* *}$ & $0.66(0.44-1.00)^{*}$ \\
Yes & & \\
Arthritis/Joint Pain & $1.00($ Reference) & $1.00($ Reference $)$ \\
No & $0.97(0.79-1.19)$ & $0.92(0.67-1.27)$ \\
Yes & $0.99(0.93-1.06)$ & $1.07(0.98-1.18)$ \\
CCI Score &
\end{tabular}

Study population includes MEPS respondents from the years 2002-2011, aged 18-64 years, who either reported being diagnosed with, or reported having an event (prescription medication, or inpatient, emergency, outpatient, or office-based provider visits) associated with, 1 or more cardiovascular disease or associated risk factor comorbidities considered in this study.

*** $P$-value $<0.001,{ }^{* *} P<0.01,{ }^{*} P<0.05$ for the difference between Continuous Medicaid vs Discontinuous-Uninsured and Continuous Medicaid vs Discontinuous-Insured groups

SE: Standard error; GED: General educational development; FPL: Federal poverty level; MSA: Metropolitan Statistical Area; SSI: Supplementary Security Income due to disability; TANF: Temporary Assistance to Needy Families program participation; HMO: Health maintenance organization; MCO: Managed care organization; CCI: Charlson Comorbidity Index score (D'Hoore adapted CCI score was calculated in this study using the 3-digit ICD-9-CM codes in MEPS). 
(OR: 1.23, 95\% CI: $1.01-1.50$ ), and individuals with intermittent employment had almost two times higher odds compared to the unemployed (OR: 1.92, 95\% CI: 1.49 2.47) of belonging to the Discontinuous-Uninsured group. When examining the Medicaid-related variables, this study found that SSI due to disability or TANF participation was associated with about 67\% lower odds (OR: 0.33, 95\% CI: $0.27-0.41$ ), and Medicaid $\mathrm{HMO} /$ managed care participation was associated with 39\% lower odds (OR: $0.61,95 \%$ CI: $0.52-0.73$ ) of experiencing Medicaid discontinuity. When examining health-related variables, this study found that individuals with respiratory illnesses had about $28 \%$ lower odds (OR: $0.72,95 \%$ CI: $0.56-0.91$ ), and those reporting fair to poor health status had about $25 \%$ higher odds (OR: $1.25,95 \%$ CI: $1.04-1.51$ ) of belonging to the Discontinuous-Uninsured group.

\section{Discontinuous-Insured}

When examining the predictors of discontinuous Medicaid coverage who also reported having other sources of health insurance coverage (Discontinuous-Insured), this study found that among the demographic characteristics, Non-Hispanic Blacks had 41\% lower odds (OR: 0.59, 95\% CI: 0.43 - 0.82), Hispanics had 35\% higher odds (OR: 0.65, 95\% CI: $0.44-0.96$ ), and married individuals had about 48\% higher odds (OR: 1.48, 95\% CI: $1.07-2.05$ ) of belonging of the Discontinuous-Insured group. In addition, as the number of family members increased by one, the odds of experiencing Medicaid discontinuity decreased by about $15 \%$ (OR: $0.85,95 \%$ CI: $0.75-0.96$ ).

Among the socioeconomic variables, this study found that individuals with higher education had higher odds compared to individuals with no high school education [OR (95\% CI) for high school/GED: 1.75 (1.25 - 2.46), and OR (95\% CI) for College degree: $3.05(2.10-4.44)]$, individuals with higher family income had higher odds compared to low-income group [OR $(95 \% \mathrm{CI})$ for $\geq 125 \%$ FPL: 2.55 (1.91 - 3.41)], and those with partial or full-year employment had higher odds [OR $(95 \% \mathrm{CI})$ for intermittent employment: 3.98 (2.87 - 5.54), and OR (95\% CI) for always employed: 3.46 (2.42 4.94)] compared to unemployed individuals of belonging to the Discontinuous-Insured group. Both the Medicaid-related variables were significant predictors with individuals who reported receiving SSI due to disability or TANF participation having $67 \%$ lower odds (OR: 0.33, 95\% CI: $0.21-0.51$ ), and those reporting Medicaid HMO/managed care participation having about 30\% lower odds (OR: $0.70,95 \% \mathrm{CI}$ : $0.53-0.92$ ) of experiencing Medicaid discontinuity. Finally, among the health-related variables, individuals who reported having respiratory illnesses had about 35\% lower odds (OR: $0.65,95 \%$ CI: $0.43-0.98$ ) of being in the Discontinuous-Insured group.

\section{DISCUSSION}

The present study findings highlight the high prevalence of Medicaid discontinuity among non-elderly adults with CVD or high-risk conditions for CVD. National estimates indicate that of the roughly 4 million individuals who reported having CVD or an associated comorbidity in the first round of the MEPS survey and Medicaid at 
any point in time during the year, a little over 1.3 million had less than full-year Medicaid coverage, over 900,000 (or $68.3 \%$ ) of which did not have any other health insurance during the year. To our knowledge, no previous study has stratified the discontinuous Medicaid group based on presence (Discontinuous-Insured) or absence (DiscontinuousUninsured) other sources of health insurance coverage. On average, the DiscontinuousUninsured group in this study was enrolled for about 6.5 months in Medicaid during a year. The instability in Medicaid coverage is further highlighted by the finding that of the 3.1 million individuals (Unweighted frequency $=4,898$ ) who had Medicaid at the beginning of the year, a little over 300,000 (or 10\%) of these individuals were uninsured at the end of the year. When this end-of-the-year insurance status was examined among individuals with family income level of $<100 \%$ of FPL, this study found that of the 1.6 million individuals who had Medicaid as the year began, over 130,000 (or 8\%) of these individuals were uninsured as the year ended. Churning in Medicaid, or multiple transitions into and out of Medicaid coverage, especially to a state of having no health insurance, are a major concern among policymakers and health policy researchers. ${ }^{8,44}$ Churning is especially concerning when it results in loss of health insurance. This study found that about $17 \%$ of the non-elderly adult population with CVD or high-risk conditions for CVD experienced more than one transition into or out of Medicaid coverage.

Hypertension, lipid disorders and diabetes, conditions considered significant risk factors for CVD, were the most prevalent chronic conditions in this population. Among the core CVD conditions, coronary artery disease, MI, and stroke - conditions that exert significant morbidity and mortality - were the most prevalent chronic conditions in this study. ${ }^{18,21}$ Hypertension has been well-established as a significant high-risk condition for CVD. ${ }^{17,21}$ This study found hypertension to be the most prevalent chronic condition among low-income individuals with CVD or high-risk conditions for CVD. Although estimates in the literature indicate the prevalence of CVD in the Medicaid population to be over 16 million as mentioned earlier, the lower numbers in this study may indicate differences in data source and inclusion criteria of first round-disease reporting to capture the chronicity of CVD and associated risk factors. The distribution of these index diseases was similar between the continuous Medicaid coverage, and both the discontinuous Medicaid groups indicating that Medicaid discontinuity, both with or without other sources of health insurance, may be predicted by factors other than differences in CVD burden.

Of the two major public health insurance programs in the U.S., Medicaid enrollees are more likely to experience discontinuity in their health care coverage in comparison to Medicare enrollees, and the prevalence and duration of coverage discontinuity are as high as private employer-sponsored insurance among the low-income groups. ${ }^{45}$ Being the primary, means-tested health insurance program for the low-income population, small increases in family incomes and assets often render individuals and families ineligible for Medicaid based on their state's Medicaid eligibility criteria. However, research has shown that several individuals and families lose Medicaid coverage despite being eligible, mainly due to the inability to periodically report changes in their income, assets, and residential status, among other information, and often not 
being able to understand the state Medicaid reenrollment procedures. ${ }^{37,46,47}$ As shown in this study a significant proportion of the discontinuous Medicaid coverage group did not report having any other source of health insurance coverage. Overwhelmingly, empirical literature suggests uninsured individuals have poor access to care, utilization of health care services, unplanned inpatient admissions, and often have poor health outcomes compared to the insured populations. ${ }^{10-14,31,32}$

Among the characteristics significantly associated with discontinuity, NonHispanic Whites had a higher likelihood of experiencing discontinuity compared to minorities, which is similar to previous findings. This may be due to the fact that minorities are more likely to be eligible for Medicaid due to differential income and assets. This is further bolstered by additional findings in this study that those with higher education, income levels, and some form of employment during the year; indicators of better socioeconomic status; have a higher likelihood of experiencing Medicaid discontinuity, which may indicate that race/ethnicity may be impacting discontinuity in conjunction with socioeconomic characteristics. Individuals residing in the southern geographic region were more likely to report having discontinuous Medicaid coverage and no other form of health insurance, which may reflect regional differences in Medicaid eligibility criteria and reenrollment procedures. ${ }^{48}$ Respondents who reported having Medicaid through managed care organizations had significantly lower likelihood of experiencing discontinuity in their Medicaid coverage which may be due to better outreach efforts to ensure continued enrollment among their plan members compared to the traditional state fee-for-service Medicaid programs. ${ }^{38}$

As of April 2015, thirty states, including the district of Columbia (D.C.) have moved forward to expand their state Medicaid programs in accordance with the regulations under the 2014 Patient Protection and Affordable Care Act (ACA), that stipulate States to provide Medicaid coverage to residents with incomes at or below 138 percent of FPL, irrespective of marital or parenthood status. ${ }^{49}$ In addition, the health law stipulated the creation of health insurance marketplaces in each state, known as the health exchanges, as a one-stop shop for state residents and employers to purchase health insurance plans. Individuals with incomes between 138 - 400 percent of FPL, who do not meet the Medicaid eligibility criteria under the expansion provision (in states not expanding Medicaid, the range is $100-400$ percent of FPL) would receive subsidies to purchase private plans from the health exchanges. ${ }^{50}$ These measures intended to expand coverage to hitherto uninsured and underinsured individuals and families may decrease the uninsured rate, but research suggests that the problems of Medicaid coverage transitions may continue to persist. Research suggests that among states not expanding Medicaid, the problems of losing Medicaid coverage to become uninsured will continue to exist, since upward income mobility would need to be significant for individuals to qualify for private insurance subsidies that will kick in above the 138 percent of FPL threshold. However, among states that do expand Medicaid, the problems of transitions from Medicaid to subsidized private insurance exchanges and/or back will also exist. ${ }^{51,52}$

When individuals lose Medicaid coverage to become uninsured, and especially when they are eligible for Medicaid, it is considered a policy failure, and the resulting 
lack of insurance may have significant adverse health outcomes. ${ }^{34}$ However, when individuals transition from one source of coverage to another, continuity of care may still be impacted, especially since different insurance plans have different network of providers, and differing benefit structures, and may cover different classes of medications and services. This study showed that over a quarter of the CVD population with discontinuous Medicaid coverage belonged to the Discontinuous-Insured group who reported having less than six months of Medicaid coverage, and were uninsured for a little over a month, on average. This may exert additional burden on individuals and families of taking out time and effort to sign up for other sources of health care coverage that includes providing the necessary documents for enrollment and may also temporarily disrupt continuity of care in this population.

Policymakers debating measures to ensure continuity in Medicaid among nonelderly adults should especially include populations with chronic and debilitating conditions such as CVD and associated risk factors in all such discussions, since the detrimental effects of coverage discontinuity and resultant disruptions in continuity of care may be amplified in such population groups. Moreover, outreach and reenrollment strategies employed by Medicaid managed care organizations can be adopted by traditional state Medicaid plans. In addition, expanding the penetration of managed care in Medicaid can also be an alternative to enable states to strike a balance between tight fiscal spending and increased and continued Medicaid enrollment. Health care providers and their staff in the meanwhile can play a major role by informing, encouraging, and if possible assisting, low-income patients with CVD, their risk factors, or disability, to signup and maintain their enrollment in Medicaid through periodic reporting as per the state's requirements. In a survey conducted for the Centers for Medicare and Medicaid Services (CMS), more than half of the respondents indicated that they would trust a doctor when making a decision to enroll in Medicaid or CHIP, and about 50 percent preferred getting Medicaid/CHIP-related information at the doctor's office. ${ }^{53}$

As with all observational research, this study is not without its limitations. Statelevel Medicaid eligibility data was not available to explore the complex and dynamic patterns of discontinuity that may differ based on the state's eligibility and reporting criteria. Nonetheless, the finding of individuals residing in the south having greater likelihood of discontinuity may have captured some of the regional variations in Medicaid policies, and future research with state-level data may better account for these differences. This study only explored associations between individual characteristics and Medicaid discontinuity, and not causality, and the findings only explore predictors of within-year discontinuous Medicaid coverage and may not capture risk factors for secular trends in Medicaid discontinuity. The regression analyses may not have adjusted for unobserved covariates that may have confounded the impact of certain risk factors on the likelihood of belonging to the Discontinuous-Uninsured or the Discontinuous-Insured groups.

Limitations of small sample size due to the focus of this study on individuals with CVD or high-risk conditions for CVD, and not the entire Medicaid population, prevented us from imputing Medicaid eligibility and obtaining robust estimates of Medicaid 
discontinuity predictors in this imputed subpopulation. Nonetheless, these findings have highlighted significant prevalence of overall discontinuous Medicaid coverage, and future studies with higher sample sizes of the CVD population can further explore predictors of Medicaid discontinuity separately among those losing eligibility due to upward income mobility, and those dropping out of Medicaid despite being eligible. Data in MEPS are self-reported, so the estimates may not exactly reflect the national estimates of Medicaid discontinuity. Researchers have speculated that most surveys underestimate the number of Medicaid enrollees due to a variety of reasons including stigma of public insurance. However, such misreporting is less of a problem for MEPS compared to other federal health surveys. ${ }^{54}$ Finally, findings from this study are representative of the noninstitutionalized civilian population and may not be applicable to individuals with prolonged hospitalizations or admitted to long-term care facilities.

\section{CONCLUSION}

The present study highlights the prevalence of Medicaid discontinuity among non-elderly adults with CVD or high-risk conditions for CVD and finds that a high proportion of individuals with less than full-year Medicaid coverage do not have any other source of health insurance. Women, individuals with higher education and income, those residing in the South census region, and those perceiving their health to be fair or poor may be at higher risk for Medicaid discontinuity. Medicaid managed care enrollees may have lower risk for Medicaid discontinuity. Outreach and reenrollment strategies employed by such managed care organizations can be adopted by state Medicaid agencies to improve Medicaid uptake, continuity and reenrollment, especially for enrollees with chronic conditions such as CVD or high-risk conditions for CVD. Discussions regarding policies to improve retention of and continuity in Medicaid must include individuals suffering from these chronic and debilitating disease conditions whose continuity of care may be adversely impacted due to such coverage instability. Low-income adults managing chronic conditions, especially in regions with stringent Medicaid eligibility regulations, must be encouraged to ensure continuity of Medicaid coverage. 


\section{CHAPTER 3. ASSOCIATIONS BETWEEN MEDICAID DISCONTINUITY, MEDICATION ADHERENCE AND MEDICATION UTILIZATION AMONG ADULTS WITH CARDIOVASCULAR DISEASE OR ASSOCIATED HIGH-RISK CONDITIONS}

\section{BACKGROUND}

Cardiovascular disease (CVD) along with conditions that are risk factors for CVD, such as diabetes and chronic kidney disease, are some of the most common causes of death, both regionally and globally. ${ }^{16,17,55}$ Randomized studies and guidelines have established the importance of lowering blood pressure, cholesterol, or blood sugar levels, along with lifestyle changes such as smoking cessation, in order to reduce morbidity and mortality associated with CVD or high-risk conditions for CVD. ${ }^{56,57}$ Nevertheless, management of the chronic risk factor conditions has been suboptimal. ${ }^{58-60}$

Pharmacotherapy is essential to appropriately treat these chronic conditions and achieve and maintain the guidelines-recommended target levels for blood pressure, blood sugar, and cholesterol. Inability to obtain or consume medications necessary to avoid risk for chronic, debilitating conditions such as CVD may have detrimental outcomes among individuals who are at risk for these conditions.

Medicaid plays a pivotal role in providing access to care for individuals with low incomes, and disabilities. Among non-elderly adult Medicaid enrollees, the prevalence of chronic conditions is high and many have complex health care needs due to existing comorbid conditions, such as mental, and respiratory illnesses. ${ }^{30}$ Prevalence of CVD or high-risk conditions for CVD among Medicaid enrollees is high; an estimated $28 \%$ of enrollees are diagnosed with CVD, $24 \%$ with high blood pressure, $17 \%$ with high cholesterol, and 9-15\% with diabetes. ${ }^{21,22}$ Due to this high chronic disease burden, adult Medicaid enrollees have high health care needs, and need the resources to effectively manage their chronic conditions. ${ }^{30}$ However, means-tested health insurance programs such as Medicaid have their own set of eligibility criteria and enrollment procedures, which vary from state to state, ${ }^{6}$ and it is critical for individuals and families to be cognizant of these policies and procedures to enroll in, and maintain eligibility, programs such as Medicaid. Small fluctuations in income levels for covered non-elderly adults due to increases in pay or work hours, would end up disqualifying individuals with incomes at or near eligibility thresholds. The rules and regulations for eligibility and enrollment between public and private health insurance are different, and therefore, individuals who have to transition between one system to another also experience gaps in insurance coverage, either due to failure to follow enrollment procedures, or being unaware of such an option, or even due to the stigma of enrolling for Medicaid or other low-income public programs. It is broadly due to these factors in this fragmented health insurance system, that individuals and families experience insurance instability and they continue to swing into and out of various types of health insurance coverage.

Instability in Medicaid coverage may not only impact utilization of prescription medications due to access issues, but may also affect adherence of these medications as 
prescribed by health care providers. Adherence to evidence-based cardiovascular medications is essential for attaining therapeutic goals, in order to achieve favorable health outcomes. ${ }^{61-64}$ Suboptimal adherence may have detrimental consequences even among non-elderly adults with multiple chronic conditions and comorbidities, who often require multiple medications to manage their disease conditions. ${ }^{65,66}$ Policies to ensure higher retention of Medicaid-eligible needy individuals and continuity in Medicaid coverage, along with seamless transition from Medicaid to other insurance coverage sources must be a priority for policymakers and other stakeholders, including health care providers. Pharmacotherapy may become one of the first casualties of loss of Medicaid coverage, since out-of-pocket costs have been found to be one of the most significant barriers to medication adherence and utilization.

Empirical studies examining the impact of lack of insurance coverage continuity on medication adherence among individuals with CVD or high-risk conditions for CVD is lacking. Among the few studies examining impact of discontinuous Medicaid coverage on medication utilization in general, or focusing on other disease conditions, there is a general agreement that having instability in coverage has an adverse impact on obtaining needed medications to manage chronic diseases. ${ }^{15,32,67}$ In intending to strengthen the literature on Medicaid coverage discontinuity by highlighting the association between discontinuous Medicaid coverage and pharmacotherapy outcomes among the CVD population, this study has the following two objectives: (1) examine whether having discontinuous Medicaid coverage is associated with poor adherence to commonly prescribed therapeutic drug classes, and (2) examine whether discontinuous Medicaid coverage is associated with low prescription drug utilization.

\section{METHODS}

\section{Data Source}

The present study is a retrospective cross-sectional comparative analysis of the Medical Expenditure Panel Survey (MEPS). MEPS is a set of large-scale surveys of families, individuals, their medical providers and employers across the United States. It is jointly sponsored by the Agency for Healthcare Research and Quality (AHRQ) and the National Center for Health Statistics (NCHS), and has been conducted annually since 1996. It has two major components; the Household Component (HC), the Insurance Component, with data on medical providers in the $\mathrm{HC}$ being supplemented by the Medical Provider Component. MEPS collects detailed information from a nationally representative sample of the civilian noninstitutionalized population of the U.S. on health services utilization and health expenditures, insurance coverage, and sources of payment.

MEPS has an overlapping panel design. Two separate panels of respondents are interviewed simultaneously during a calendar year. A new panel of sample households is selected each year, and data for each panel are collected for two calendar years. The two years of data for each panel are collected in five rounds of interviews that take place over 
a two and a half year period. This provides continuous and current estimates of health care expenditures at both the person and household level for two panels for each calendar year. By combining data from the overlapping panels, these annual files provide nearly double the sample size of individual panels and cover the entire calendar year for each respondent. Although all MEPS data are reported by respondents during computerassisted personal interviewing, further detailed health service use data, including on prescription drugs, are collected from a sample of providers with respondents' permission. ${ }^{39}$

When obtaining national estimates from surveys such as MEPS, appropriate sample size is critical to obtain reliable estimates, since estimates of some population subgroups may vary from year to year. The advantage of survey such as MEPS is that they allow for several years' of data to be pooled together in order to increase the sample size and improve the precision of estimates. The pooling of the different years of data was conducted in accordance with the guidelines provided by MEPS, and data for the years $2002-2011$ were pooled together to allow for a sufficiently large sample size to ensure reliable estimates.

\section{Study Population}

The broad classification of "cardiovascular disease (CVD) and associated comorbidities" in this study consisted for the following disease conditions: acute myocardial infarction (Clinical Classification code (CCC): 100), coronary artery disease (CCC: 101), congestive heart failure (CCC: 108), peripheral and visceral atherosclerosis (CCCs: 114, 115, 116), stroke (CCCs: 109, 112), hypertension (CCC: 098), lipid disorders (CCC: 053), and diabetes (CCC: 049, 050). CCC aggregates conditions and procedures into mutually exclusive and clinically homogeneous categories, using Clinical Classification Software. ${ }^{40}$ When examining utilization of prescription medications in the form of number of medication refills, the study population included respondents who were: (1) aged 18 to 64 years, (2) reported either being diagnosed with CVD or high-risk conditions for CVD for CVD in their first round of the MEPS survey, or reported having an event (an inpatient, emergency, outpatient, or office-based provider visits, or a prescription medication) associated with one or more of the aforementioned disease conditions at any point of time during the survey, (3) reported having had Medicaid coverage at any point of time during the survey year, and (4) had positive person weights, and were interviewed for all five rounds of the 2-year MEPS survey interviews. When examining adherence to commonly prescribed medication classes for managing the CVD and associated risk factor comorbidities listed above, an additional inclusion criteria was considered: (5) respondents who had one or more fills or refills for the following therapeutic drug classes - angiotensin converting enzymes inhibitors (ACEI), angiotensin II receptor blockers (ARB), beta blockers, calcium channel blockers, diuretics, antihypertensive drug combinations, HMG-CoA reductase inhibitors (statins), sulfonylureas, and biguanides. 
Respondents who reported having Medicare, or pregnancy were excluded from this study. Pregnant women with incomes at or below 133 percent of the federal poverty level are eligible for Medicaid coverage throughout the pregnancy; however, the coverage ceases 60 days postpartum. ${ }^{68}$ Low-income non-elderly adults with disabilities are also eligible for Medicare if they have received monthly social security disability income for two years. Most coverage benefits for prescription drugs for dual eligible beneficiaries are covered by Medicare Part D, instead of Medicaid. As a result, these two population groups were excluded from this study.

Individuals who have less than a year of Medicaid coverage and experience within-year Medicaid discontinuity may not be a homogenous group, especially when they report having other forms of insurance coverage during the year. In order to account for these differences, individuals in the study population who reported having $<12$ months of Medicaid coverage during a survey year were divided into two groups: (1) individuals with $<12$ months of Medicaid coverage who did not report any other sources of health insurance during the survey year, designated in this study as 'DiscontinuousUninsured', and (2) individuals with $<12$ months of Medicaid coverage who also reported having other source(s) of health insurance during the survey year (either private, Tricare, or any hospital- or physician group-based health insurance), designated in this study as 'Discontinuous-Insured'. Individuals in the study population who reported having Medicaid coverage during all 12 months of a survey year were classified as having continuous Medicaid coverage, which was the comparison group and designated as 'Continuous Medicaid' in this study.

\section{Study Variables}

Our first objective was to examine whether Medicaid discontinuity was associated with adherence to commonly prescribed classes of medications among non-elderly adults with CVD or its associated risk factor comorbidities. The primary outcomes of interest was the binary indicator for medication adherence measured as the medication possession ratio (MPR), or the proportion of days during the survey year during which respondents had medications on hand. In the subsequent section, we describe how adherence was measured for this study. The second objective was to examine whether Medicaid discontinuity impacts medication utilization. The primary outcome of interest for this objective was the raw number of refills for all medications reported by the study respondents. We divided this utilization measure into all-cause, and disease-specific medication utilization, i.e. number of refills for all medications reported by the study respondents in the survey year (all-cause), and the number of refills for medications specifically prescribed for CVD or high-risk conditions for CVD for CVD that were examined in this study (disease-specific).

All regression models included the following classes of covariates:-

1) Demographic covariates: age (in years), gender (female vs. male), race/ethnicity (Non-Hispanic White vs. Non-Hispanic Black, Hispanic, and other races), marital 
status (not married vs. married), family size $(\mathrm{N})$, and geographic residence (Northeast vs. Midwest, South, and West).

2) Socioeconomic covariates: education (less than high school vs. high school/GED, and Bachelor's or higher), income as a percentage of the federal poverty level (FPL) $(<100 \%$ FPL vs. $100-<125 \%$ FPL, and $\geq 125 \%$ FPL), employment (always unemployed vs. intermittent employment, and always employed), binary indicator for Metropolitan Statistical Area (MSA) residence.

3) Medicaid-related covariates: binary indicator for Supplementary Security Income (SSI) for disability receipt or participation in the Temporary Assistance for Needy Families program, and a binary indicator for Medicaid HMO/Managed Care participation.

4) Health-related covariates: perceived health status (excellent/very good/good vs. fair/poor), perceived mental health status (excellent/very good/good vs. fair/poor) binary indicator for mental illness/substance abuse diagnosis, binary indicator for respiratory diseases diagnosis, binary indicator for arthritis/joint pain diagnosis, and Charlson Comorbidity Index (CCI) score.

When examining medication adherence, the regression models included the following additional covariates:

5) Medication-related covariates: number of unique prescription medications reported by the study respondents $(\mathrm{N})$, and a binary indicator for whether individuals had a 90 days' supply of medications.

All regression models included the year fixed effects to capture any macro-level variations during the study period. SAS 9.3 and STATA 13 were used.

\section{Measurement of Adherence}

The measurement of adherence in this study involved several intermediate steps. From the Prescribed Medicines files in MEPS, this study used the drug refill records for each study respondent, to estimate adherence to each medication class during the year when the respondents had refills for any medication from that class of medication. Of the two most common measures of adherence, medication possession ratio (MPR) and proportion of days covered (PDC), this study used the MPR measure. ${ }^{69,70}$ Using an interval-based MPR measure approach, the denominator for this measure was the number of days between the index date, which was first day of the round in which the first fill or refill of a class of drug (e.g. beta blockers) was reported, and the last day of the survey year, i.e. December $31^{\text {st }}$. The numerator is the total number of days covered by drug fills during the denominator duration, i.e. the total days' supply for that particular class of medication within the follow-up (denominator) period. Equation 3-1 displays the average MPR estimation:

$$
\frac{\sum \quad \text { total Rx days of supply }}{\text { Interval }_{n}(\text { round date for first Rx during year - December 31) }} / n
$$


As mentioned earlier, the proportion of the total days' supply for a therapeutic class of medication was estimated within the follow-up interval reported by an individual. Depending on the number of therapeutic classes reported by each individual, the MPR was estimated for each class and summed, and then divided by the number of therapeutic classes reported by individuals (n) to obtain an average MPR measure for that individual. This enables capturing non-adherence, since any discontinuation in the drug refill will be captured in the calculation as it tracks adherence till the end of the duration and not the last drug refill during the measurement period, as is common with the standard measures of MPR. ${ }^{69}$ All MPR measures were expressed as a percentage and capped at $100 \%$ (or 1.0). Drugs that were dispensed within a therapeutic class (e.g. switching from carvedilol to propranolol among beta blockers) were considered interchangeable. ${ }^{69} \mathrm{ACEI}$ and ARBs were considered a single class for this study.

More than $90 \%$ of index refills had occurred in the first two rounds of the calendar year. The drug class-specific MPRs that were obtained were summed, and an overall average MPR measure was calculated to summarize adherence to all drug classes an individual was taking. In order to make policy-relevant conclusions about the impact of insurance discontinuity on medication adherence, the continuous MPR measure was transformed into a binary MPR measure, with a cutoff of $80 \%(0.8)$ or more signifying adequate adherence to prescribed medications examined in this study. The main outcome variable was the overall binary MPR measure. Class-specific MPR adherence measures were examined in secondary analyses.

Prior to 2010, MEPS did not report data on days' supply for the prescription medications reported by the respondents during the survey year. Previous studies have overcome this shortcoming, by developing algorithms for days' supply based on the quantity of drug dispensed using data from administrative claims, or other similar sources. A similar approach was applied; however, rather than using an external source of data, the 2010 and 2011 MEPS prescription medication data that had the days' supply information was used to develop an algorithm that approximates days' supply for previous years in MEPS based on their quantity prescribed/dispensed, which was available for all the 10 years in MEPS. The most frequent number of supply days furnished by each level of dispensed quantity was first identified, and found that 30 , and 90 days' supply were the most common patterns observed for most quantities dispensed. After deriving a method to smoothly approximate the distribution of the days' supply, the days of supply were categorized using different sets of categories. The third categorization algorithm was found to have the highest correlation with the actual MPR measures, both continuous and binary, which were calculated using the days' supply variables from 2011 and 2010 years of MEPS data, separately as displayed in Table 3-1 and Table 3-2. Finally, using this algorithm, the MPR adherence measures for all years prior to 2010 were calculated to obtain overall estimates of medication adherence. 
Table 3-1. Refill Days of Supply Algorithms and MPR Validation in 2011 Sample $(\mathrm{N}=\mathbf{5 , 4 7 5 )}$

\begin{tabular}{|c|c|c|c|c|c|}
\hline \multirow[b]{2}{*}{ Categorization } & \multirow[b]{2}{*}{$\begin{array}{l}\text { Quantity } \\
\text { Dispensed }\end{array}$} & \multirow[b]{2}{*}{$\begin{array}{c}\text { Corresponding } \\
\text { Days' Supply } \\
\text { Algorithm }\end{array}$} & \multirow[b]{2}{*}{$\begin{array}{c}\text { Continuous MPR } \\
\text { Measure } \\
\text { Pearson's } \\
\text { Correlation }\end{array}$} & \multicolumn{2}{|c|}{ Binary MPR Measure } \\
\hline & & & & $\begin{array}{c}\text { Observed } \\
\text { Agreement (\%) }\end{array}$ & к-Statistic \\
\hline Categorization 1 & $\begin{array}{l}\leq 75 \\
>75\end{array}$ & $\begin{array}{l}30 \\
90\end{array}$ & 0.868 & 0.925 & $0.841 * * *$ \\
\hline Categorization 2 & $\begin{array}{c}\leq 50 \\
>50-\leq 80 \\
>80\end{array}$ & $\begin{array}{l}30 \\
60 \\
90\end{array}$ & 0.881 & 0.931 & $0.854 * * *$ \\
\hline Categorization 3 & $\begin{array}{c}\leq 44 \\
45 \\
>45-\leq 75 \\
>75-\leq 119 \\
120 \\
>120\end{array}$ & $\begin{array}{l}30 \\
90 \\
30 \\
90 \\
30 \\
90\end{array}$ & 0.889 & 0.934 & $0.864 * * *$ \\
\hline
\end{tabular}

MPR: Medication Possession Ratio

$* * * P<0.001$. The null hypothesis for estimating kappa statistic is that there is no agreement between the two discrete measures of MPR. 
Table 3-2. Refill Days of Supply Algorithms and MPR Validation in 2010 Sample $(\mathrm{N}=5,058)$

\begin{tabular}{|c|c|c|c|c|c|}
\hline \multirow[b]{2}{*}{ Categorization } & \multirow[b]{2}{*}{$\begin{array}{c}\text { Quantity } \\
\text { Dispensed }\end{array}$} & \multirow[b]{2}{*}{$\begin{array}{c}\text { Corresponding } \\
\text { Days'Supply } \\
\text { Algorithm }\end{array}$} & \multirow[b]{2}{*}{$\begin{array}{c}\text { Continuous MPR } \\
\text { Measure } \\
\text { Pearson's } \\
\text { Correlation }\end{array}$} & \multicolumn{2}{|c|}{ Binary MPR Measure } \\
\hline & & & & $\begin{array}{c}\text { Observed } \\
\text { Agreement (\%) }\end{array}$ & к-Statistic \\
\hline Categorization 1 & $\begin{array}{l}\leq 75 \\
>75\end{array}$ & $\begin{array}{l}30 \\
90\end{array}$ & 0.835 & 0.918 & $0.827 * * *$ \\
\hline Categorization 2 & $\begin{array}{c}\quad \leq 50 \\
>50-\leq 80 \\
>80\end{array}$ & $\begin{array}{l}30 \\
60 \\
90\end{array}$ & 0.856 & 0.876 & $0.747 * * *$ \\
\hline Categorization 3 & $\begin{array}{c}\leq 44 \\
45 \\
>45-\leq 75 \\
>75-\leq 119 \\
120 \\
>120\end{array}$ & $\begin{array}{l}30 \\
90 \\
30 \\
90 \\
30 \\
90\end{array}$ & 0.868 & 0.924 & $0.841 * * *$ \\
\hline
\end{tabular}

MPR: Medication Possession Ratio

$* * * P<0.001$. The null hypothesis for estimating kappa statistic is that there is no agreement between the two discrete measures of MPR. 


\section{Statistical Analysis}

Bivariate statistics were conducted when examining the characteristics of the study population. Survey-weighted proportions of the use of different classes of medications examined in this study were calculated. When examining medication adherence, Survey-weighted multivariate logistic regression analyses were conducted. Equation 3-2 and Equation 3-3 depict the logistic regression models to examine adherence among both the Discontinuous-Uninsured, and the Discontinuous-Insured groups relative to the Continuous Medicaid insurance group.

$$
\begin{aligned}
& \operatorname{Pr}[\mathrm{MPR} \geq 0.8 \mid \mathrm{x}]=\mathrm{F}^{-1}\left(\hat{\alpha}+\hat{\beta}_{1} \text {. Discontinuous }- \text { Uninsured }+\sum_{k=1}^{K} \hat{\beta}_{k} X_{k}\right) \\
& \operatorname{Pr}[\mathrm{MPR} \geq 0.8 \mid \mathrm{x}]=\mathrm{F}^{-1}\left(\hat{\alpha}+\hat{\beta}_{1} . \text { Discontinuous }- \text { Insured }+\sum_{k=1}^{K} \hat{\beta}_{k} X_{k}\right)
\end{aligned}
$$

Medication adherence is modeled as a dichotomous outcome variable as a function of Medicaid discontinuity as well as the demographic, socioeconomic, eligibility, health-related, and medication-related covariates mentioned above. $\mathrm{F}^{-1}$ denotes the inverse of the cumulative standard logistic distribution function that relates the outcome on the probability scale to the covariates. $\operatorname{Pr}[$ ] denotes the population average probability of adherence to medications conditional on the covariates denoted by $\mathrm{x}$. Discontinuous-Uninsured and Discontinuous-Insured are binary indicator variables for the main independent variable of interest, that equals one for individuals having discontinuous annual Medicaid coverage without any other sources of insurance and with other sources of insurance during the year, respectively, and equals zero for the comparison group of individuals with continuous Medicaid coverage. $X$ denotes the vector of aforementioned individual characteristics that predict Medicaid discontinuity in the two population groups of interest.

When examining all-cause and disease-specific medication utilization using the number of prescription drug fills as the outcome, the negative binomial regression model was utilized due to the skeweness in the distribution exhibited by the outcomes measures. Appendix A, Figures A-1 to A-4, display the distribution in the all-cause and diseasespecific prescription drug utilization outcome measures. It is a type of generalized linear model where the dependent variable $Y$ is a count of the number of times an event occurs (in this case, the number of drug fills). A convenient parameterization of the negative binomial distribution is denoted by Equation 3-4. ${ }^{71}$

$$
p(y)=P(Y=y)=\frac{\Gamma\left(y+\frac{1}{\alpha}\right)}{\Gamma(y+1) \Gamma\left(\frac{1}{\alpha}\right)}\left(\frac{1}{1+\alpha \mu}\right)^{1 / \alpha}\left(\frac{\alpha \mu}{1+\alpha \mu}\right)^{y} \quad(\mathbf{E q} \cdot 3-4)
$$

In Equation 3-4, $\mu>0$ is the mean of $Y$, which is the count variable indicating the number of prescription drug fills (either all-cause, or disease-specific) and $\alpha>0$ is the heterogeneity parameter. It can be derived as a Poisson-gamma mixture, or as the number of failures before the $(1 / \alpha)^{\text {th }}$ success. A simpler depiction of the traditional negative binomial regression model, the NB2 model is depicted is depicted in Equation 3-5 and Equation 3-6 as follows: 


$$
\begin{aligned}
& \ln \mu_{\text {all-cause }}=\beta_{0}+\beta_{1} \text { Discontinuous }- \text { Uninsured }+\beta_{2} \cdot x_{2}+\cdots+\beta_{k} \cdot x_{k} \text { (Eq. 3-5) } \\
& \ln \mu_{\text {disease-specific }}=\beta_{0}+\beta_{1} \text { Discontinuous }- \text { Uninsured }+\beta_{2} \cdot x_{2}+\cdots+\beta_{k} \cdot x_{k}
\end{aligned}
$$

Apart from the main indicator variables for the Discontinuous-Uninsured group, the covariates $x_{2}$ to $x_{k}$ are known, and the population regression coefficients $\beta_{0}$ to $\beta_{k}$ are to be estimated. The two equations above depict the negative binomial regression models to estimate the differences in all-cause prescription drug utilization (Equation 3-5), and disease-specific prescription drug utilization (Equation 3-6) between the Continuous Medicaid, and Discontinuous-Uninsured groups. Similar regression models (not shown here) were run to estimate differences in prescription drug utilization between the Continuous Medicaid and Discontinuous-Insured groups. The coefficient of interest for the Discontinuous-Uninsured is the measure of differences in the utilization of overall, and disease-specific drug utilization. Because of the functional form of the negative binomial regression, the effect size of a factor is the antilog of its coefficient. This estimated coefficients are transformed to obtain the incidence rate ratio (IRR). An IRR of less than one for the discontinuous Medicaid groups would suggest lower medication utilization among them when compared to their counterparts with continuous Medicaid coverage.

In addition to obtaining IRRs, this study further estimated the average marginal effect to determine the predicted difference in the number of all-cause and diseasespecific prescription drug fills between the continuous and discontinuous Medicaid groups. The marginal effects measure the discrete change in the outcome variable, i.e. for a binary independent variable, how do predicted probabilities change as the binary independent variable changes from 0 (Continuous Medicaid) to 1 (DiscontinuousUninsured, or Discontinuous-Insured). ${ }^{72}$ In order to obtain these marginal effects, after developing and running the multivariate negative binomial regression model, the estimated coefficients of the model were used to calculate two predicted prescription drug fills for all individuals in the study population. The first predicted utilization assumed all individuals had discontinuous Medicaid coverage (by setting the Medicaid discontinuity indicator variable to 1), and the second predicted utilization assumed all individual to have continuous full-year Medicaid coverage (by setting the Medicaid discontinuity indicator variable to 0 ). The average per-person difference in prescription drug fills attributable to Medicaid coverage was estimated by obtaining the difference in predicted drug utilization for each person and computing the weighted average of the difference across the entire study sample. All data analyses were conducted using SAS 9.3 and STATA 13 to account for the complex survey design of MEPS. 


\section{RESULTS}

\section{Characteristics of the Study Populations}

The characteristics of the study population when examining adherence to commonly prescribed medications to manage CVD or high-risk conditions for CVD, and when examining utilization of prescription medications, are displayed in Table 3-3 and Table 3-4, respectively. The study sample when examining overall medication adherence included 3,210 respondents in the Continuous Medicaid coverage group (nationally representative of about 2 million individuals), 1,088 individuals in the DiscontinuousUninsured group (nationally representative of over 700,000 individuals), and 388 individuals in the Discontinuous-Insured group (nationally representative of over 300,000 individuals). When examining utilization of prescription medications in the study population, the study sample included 3,926 respondents in the Continuous Medicaid coverage group (nationally representative of about 2.4 million individuals), 1,407 individuals in the Discontinuous-Uninsured group (nationally representative of over 900,000 individuals), and 493 individuals in the Discontinuous-Insured group (nationally representative of over 400,000 individuals). The difference being, when examining medication adherence there was an additional inclusion criteria; the study population consisted of individuals who reported taking one or more of the eight classes of medications for which adherence was examined.

When examining medication adherence (Table 3-3), in comparison to the Continuous Medicaid group, individuals in the Discontinuous-Uninsured group were less likely to belong to other race groups $(P=0.016)$, and more likely to be married $(P<0.001)$, and were more likely to belong to the South geographic region $(P=0.010)$. Among the socioeconomic covariates, this study found that respondents were more likely to have completed high school/GED or college $(P=0.006)$, were more likely to have family incomes $\geq 125 \%$ FPL $(P<0.001)$, and more likely to have intermittent, as well as continuous full-year employment $(P<0.001)$. Respondents were less likely to be eligible for SSI due to disability or participate in TANF $(P<0.001)$, and less likely to report Medicaid HMO/managed care $(P<0.001)$. Among the health-related variables, respondents were less likely to report having fair or poor perceived mental health status ( $P=0.001)$, less likely to report having mental /substance abuse illnesses $(P=0.014)$, and less likely to report having respiratory illnesses $(P<0.001)$. The mean CCI score was not significantly different between the two groups $(P=0.181)$. When examining the medication-related variables, this study found that although the Discontinuous-Uninsured group on average were prescribed fewer medications than the Continuous Medicaid group (7.6 vs. 9.1 medications, $P<0.001)$, and both groups had similar proportion of 90 days' supply for their medications $(P=0.242)$.

When comparing the Discontinuous-Insured group to the Continuous Medicaid group, this study found that among the demographic covariates, individuals in the Discontinuous-Insured group were more likely to be younger ( $P=0.034)$, were less likely to belong to minority race/ethnicity groups $(P<0.001)$, more likely to be married 
Table 3-3. Study Population Characteristics when Examining Medication Adherence

\begin{tabular}{|c|c|c|c|c|c|c|c|c|}
\hline \multirow[b]{2}{*}{ Characteristics } & \multicolumn{2}{|c|}{ Continuous Medicaid } & \multicolumn{3}{|c|}{ Discontinuous-Uninsured } & \multicolumn{3}{|c|}{ Discontinuous-Insured } \\
\hline & Weighted N & $\%(\mathrm{SE})$ & Weighted N & $\%(\mathrm{SE})$ & $P$-value ${ }^{\dagger}$ & Weighted N & $\%(\mathrm{SE})$ & $P$-value ${ }^{\dagger \dagger}$ \\
\hline DEMOGRAPHIC & & & & & & & & \\
\hline Age & & & & & 0.876 & & & 0.014 \\
\hline $18-34$ years & 248,650 & $12.5(0.9)$ & 93,295 & $13.1(1.3)$ & & 65,519 & $19.6(2.4)$ & \\
\hline $35-49$ years & 669,245 & $33.8(1.3)$ & 242,313 & $34.2(2.1)$ & & 100,718 & $30.1(3.0)$ & \\
\hline $50-64$ years & $1,065,905$ & $53.7(1.4)$ & 373,450 & $52.7(2.3)$ & & 168,190 & $50.3(3.3)$ & \\
\hline Gender & & & & & 0.939 & & & 0.190 \\
\hline Female & $1,334,759$ & $67.3(1.3)$ & 475,870 & $67.1(2.0)$ & & 210,679 & $63.0(3.2)$ & \\
\hline Race/Ethnicity & & & & & 0.016 & & & $<0.001$ \\
\hline Non-Hispanic White & 894,528 & $45.1(1.9)$ & 336,390 & $47.4(2.3)$ & & 211,937 & $63.4(3.3)$ & \\
\hline Non-Hispanic Black & 562,325 & $28.3(1.5)$ & 183,702 & $25.9(1.9)$ & & 64,835 & $19.4(2.2)$ & \\
\hline Hispanic & 341,418 & $17.2(1.2)$ & 145,309 & $20.5(1.6)$ & & 34,802 & $10.4(1.6)$ & \\
\hline Others & 185,529 & $9.4(0.9)$ & 43,657 & $6.2(1.0)$ & & 22,852 & $6.8(1.5)$ & \\
\hline Marital Status & & & & & $<0.001$ & & & $<0.001$ \\
\hline Married & 602,503 & $30.4(1.4)$ & 271,354 & $38.3(2.1)$ & & 154,101 & $46.1(3.6)$ & \\
\hline Region & & & & & 0.064 & & & 0.040 \\
\hline Northeast & 481,061 & $24.3(1.7)$ & 141,392 & $19.9(2.0)$ & & 82,125 & $24.5(3.1)$ & \\
\hline Midwest & 393,671 & $19.8(1.4)$ & 139,468 & $19.7(1.8)$ & & 92,872 & $27.8(4.0)$ & \\
\hline South & 607,916 & $30.6(1.7)$ & 255,996 & $36.1(2.0)$ & & 76,828 & $23.0(2.7)$ & \\
\hline West & 501,152 & $25.3(1.7)$ & 172,201 & $24.3(2.0)$ & & 82,602 & $24.7(3.4)$ & \\
\hline Family Size Mean (SE) & $2.7(0.1)$ & & $2.8(0.1)$ & & 0.532 & $2.8(0.1)$ & & 0.449 \\
\hline
\end{tabular}


Table 3-3. (Continued)

\begin{tabular}{|c|c|c|c|c|c|c|c|c|}
\hline \multirow[b]{2}{*}{ Characteristics } & \multicolumn{2}{|c|}{ Continuous Medicaid } & \multicolumn{3}{|c|}{ Discontinuous-Uninsured } & \multicolumn{3}{|c|}{ Discontinuous-Insured } \\
\hline & $\begin{array}{c}\text { Weighted } \\
\text { N }\end{array}$ & $\%(\mathrm{SE})$ & Weighted N & $\%(\mathrm{SE})$ & $P$-value ${ }^{\dagger}$ & Weighted N & $\%(\mathrm{SE})$ & $P$-value ${ }^{\dagger \dagger}$ \\
\hline \multicolumn{9}{|l|}{ SOCIOECONOMIC } \\
\hline Education & & & & & 0.006 & & & $<0.001$ \\
\hline$<$ High School & 776,408 & $39.5(1.5)$ & 226,415 & $32.2(1.9)$ & & 51,716 & $15.5(2.1)$ & \\
\hline High School/GED & 997,819 & $50.7(1.4)$ & 392,411 & $55.7(2.2)$ & & 176,153 & $52.8(3.6)$ & \\
\hline College Degree & 193,484 & $9.8(0.8)$ & 85248 & $12.1(1.6)$ & & 105,631 & $31.7(3.6)$ & \\
\hline Income & & & & & $<0.001$ & & & $<0.001$ \\
\hline$<100 \%$ FPL & $1,113,782$ & $56.1(1.3)$ & 324,544 & $45.8(2.0)$ & & 74,686 & $22.3(2.3)$ & \\
\hline $100-<125 \% \mathrm{FPL}$ & 185,942 & $9.4(0.6)$ & 69,730 & $9.8(1.2)$ & & 24,692 & $7.4(1.5)$ & \\
\hline$\geq 125 \% \mathrm{FPL}$ & 684,076 & $34.5(1.2)$ & 314,784 & $44.4(2.1)$ & & 235,048 & $70.3(2.8)$ & \\
\hline Employment & & & & & $<0.001$ & & & $<0.001$ \\
\hline Always Unemployed & $1,468,299$ & $74.0(1.3)$ & 399,549 & $56.3(2.0)$ & & 91,161 & $27.3(2.5)$ & \\
\hline Unstable Employment & 231,945 & $11.7(0.9)$ & 165,706 & $23.4(1.9)$ & & 102,142 & $30.5(2.9)$ & \\
\hline Always Employed & 283,555 & $14.3(1.0)$ & 143,803 & $20.3(1.5)$ & & 141,123 & $42.2(3.2)$ & \\
\hline MSA & $1,564,442$ & $78.9(1.8)$ & 552,171 & $77.9(2.0)$ & 0.651 & 267,784 & $80.1(2.7)$ & 0.696 \\
\hline \multicolumn{9}{|l|}{ MEDICAID-RELATED } \\
\hline SSI/TANF & 852,158 & $43.0(1.5)$ & 127,179 & $17.9(1.5)$ & $<0.001$ & 30,671 & $9.2(1.8)$ & $<0.001$ \\
\hline Medicaid HMO/MCO & $1,139,857$ & $60.4(1.6)$ & 354,416 & $50.3(2.1)$ & $<0.001$ & 180,662 & $54.0(3.4)$ & 0.077 \\
\hline
\end{tabular}


Table 3-3. (Continued)

\begin{tabular}{|c|c|c|c|c|c|c|c|c|}
\hline \multirow[b]{2}{*}{ Characteristics } & \multicolumn{2}{|c|}{ Continuous Medicaid } & \multicolumn{3}{|c|}{ Discontinuous-Uninsured } & \multicolumn{3}{|c|}{ Discontinuous-Insured } \\
\hline & Weighted N & $\%(\mathrm{SE})$ & Weighted N & $\%(\mathrm{SE})$ & $P$-value ${ }^{\dagger}$ & Weighted N & $\%(\mathrm{SE})$ & $P$-value ${ }^{\dagger \dagger}$ \\
\hline HEALTH-RELATED & & & & & & & & \\
\hline Perceived Health Status & & & & & 0.050 & & & $<0.001$ \\
\hline Fair to Poor & $1,112,169$ & $56.1(1.4)$ & 366,568 & $51.7(2.1)$ & & 140,242 & $41.9(2.8)$ & \\
\hline Perceived Mental Health & & & & & 0.001 & & & $<0.001$ \\
\hline Fair to Poor & 670,915 & $33.9(1.4)$ & 189,140 & $26.7(1.9)$ & & 62,634 & $18.7(2.4)$ & \\
\hline $\begin{array}{l}\text { CVD or high-risk } \\
\text { conditions for CVD }\end{array}$ & & & & & 0.998 & & & 0.066 \\
\hline$\leq 1$ & $1,281,387$ & $64.6(1.3)$ & 457,590 & $64.5(2.0)$ & & 240,536 & $71.9(2.7)$ & \\
\hline 2 & 400,899 & $20.2(0.9)$ & 144,019 & $20.3(1.6)$ & & 58,036 & $17.4(2.5)$ & \\
\hline$\geq 3$ & 301,513 & $15.2(1.0)$ & 107,449 & $15.2(1.5)$ & & 35,854 & $10.7(1.5)$ & \\
\hline $\begin{array}{l}\text { Mental/Substance Abuse } \\
\text { Illnesses }\end{array}$ & 606,104 & $30.6(1.4)$ & 177,733 & $25.1(1.8)$ & 0.014 & 94,737 & $28.3(2.8)$ & 0.463 \\
\hline Respiratory Illnesses & 394,992 & $19.9(1.2)$ & 87,482 & $12.3(1.2)$ & $<0.001$ & 32,380 & $9.7(1.8)$ & $<0.001$ \\
\hline Arthritis/Joint Pain & 693,646 & $35.0(1.4)$ & 221,951 & $31.3(1.9)$ & 0.073 & 97,019 & $29.0(3.0)$ & 0.073 \\
\hline CCI Score Mean (SE) & $1.2(0.0)$ & & $1.1(0.1)$ & & 0.181 & $1.1(0.1)$ & & 0.058 \\
\hline Population Size & $1,983,799$ & $65.5(1.1)$ & 709,058 & $23.4(0.9)$ & & 334,426 & $11.1(0.8)$ & \\
\hline
\end{tabular}




\section{Table 3-3. (Continued)}

Study population includes MEPS respondents from the years 2002-2011, aged 18-64 years, who either reported being diagnosed with or having an event (prescription medication, or inpatient, emergency, outpatient, or office-based provider visits) associated with CVD or highrisk conditions for CVD, had $\leq 1$ month of Medicaid coverage during the survey year, and who consumed one or more medications from the following therapeutic drug classes: ACEI/ARB, beta blockers, calcium channel blockers, diuretics, anti-HTN combinations, statins, sulfonylureas, and biguanides.

${ }^{\dagger} P$-value for the difference between Discontinuous-Uninsured and Continuous Medicaid groups. ${ }^{\dagger \dagger} P$-value for the difference between Discontinuous-Insured and Continuous Medicaid groups. $P$-values in bold indicate statistically significant difference below the threshold value of $P<0.05$.

SE: Standard error; GED: General educational development; FPL: Federal poverty level; MSA: Metropolitan Statistical Area; SSI:

Supplementary Security Income due to disability; TANF: Temporary Assistance to Needy Families program participation; HMO: Health maintenance organization; MCO: Managed care organization; CCI: Charlson Comorbidity Index score (D'Hoore adapted CCI score was calculated in this study using the 3-digit ICD-9-CM codes in MEPS). 
Table 3-4. Study Population Characteristics when Examining Medication Utilization

\begin{tabular}{|c|c|c|c|c|c|c|c|c|}
\hline \multirow[b]{2}{*}{ Characteristics } & \multicolumn{2}{|c|}{ Continuous Medicaid } & \multicolumn{3}{|c|}{ Discontinuous-Uninsured } & \multicolumn{3}{|c|}{ Discontinuous-Insured } \\
\hline & Weighted N & $\%$ (SE) & Weighted N & $\%(\mathrm{SE})$ & $P$-value ${ }^{\dagger}$ & Weighted N & $\%(\mathrm{SE})$ & $P$-value ${ }^{\dagger \dagger}$ \\
\hline DEMOGRAPHIC & & & & & & & & \\
\hline Age & & & & & 0.385 & & & 0.013 \\
\hline $18-34$ years & 388,425 & $15.9(1.0)$ & 165,340 & $18.1(1.3)$ & & 98,188 & $23.1(2.3)$ & \\
\hline $35-49$ years & 875,809 & $35.9(1.2)$ & 324,653 & $35.5(1.8)$ & & 141,109 & $33.3(2.7)$ & \\
\hline $50-64$ years & $1,173,144$ & $48.2(1.3)$ & 423,933 & $46.4(2.0)$ & & 184,968 & $43.6(3.0)$ & \\
\hline Gender & & & & & 0.491 & & & 0.169 \\
\hline Female & $1,632,006$ & $67.0(1.2)$ & 624,410 & $68.3(1.8)$ & & 267,502 & $63.1(2.8)$ & \\
\hline Race/Ethnicity & & & & & $<0.001$ & & & $<0.001$ \\
\hline Non-Hispanic White & $1,094,002$ & $44.9(1.8)$ & 442,534 & $48.4(2.1)$ & & 256,231 & $60.4(3.2)$ & \\
\hline Non-Hispanic Black & 666,358 & $27.3(1.5)$ & 219,033 & $24.0(1.6)$ & & 79,936 & $18.8(2.0)$ & \\
\hline Hispanic & 445,601 & $18.3(1.2)$ & 202,106 & $22.1(1.5)$ & & 50,704 & $12.0(1.6)$ & \\
\hline Others & 231,416 & $9.5(0.9)$ & 50,253 & $5.5(0.9)$ & & 37,393 & $8.8(1.7)$ & \\
\hline Marital Status & & & & & $<0.001$ & & & $<0.001$ \\
\hline Married & 727,457 & $29.9(1.3)$ & 344,150 & $37.7(1.8)$ & & 184,414 & $43.5(3.2)$ & \\
\hline Region & & & & & 0.049 & & & 0.023 \\
\hline Northeast & 608,070 & $25.0(1.6)$ & 187,785 & $20.5(1.7)$ & & 104,394 & $24.6(2.9)$ & \\
\hline Midwest & 477,401 & $19.6(1.4)$ & 184,710 & $20.2(1.7)$ & & 115,146 & $27.2(3.2)$ & \\
\hline South & 727,520 & $29.8(1.6)$ & 315,073 & $34.5(1.8)$ & & 98,072 & $23.1(2.3)$ & \\
\hline West & 624,386 & $25.6(1.7)$ & 226,358 & $24.8(1.9)$ & & 106,652 & $25.1(3.0)$ & \\
\hline Family Size Mean (SE) & $2.8(0.1)$ & & $2.9(0.1)$ & & 0.409 & $2.8(0.1)$ & & 0.976 \\
\hline
\end{tabular}


Table 3-4. (Continued)

\begin{tabular}{|c|c|c|c|c|c|c|c|c|}
\hline \multirow[b]{2}{*}{ Characteristics } & \multicolumn{2}{|c|}{ Continuous Medicaid } & \multicolumn{3}{|c|}{ Discontinuous-Uninsured } & \multicolumn{3}{|c|}{ Discontinuous-Insured } \\
\hline & Weighted N & $\%(\mathrm{SE})$ & Weighted N & $\%(\mathrm{SE})$ & $P$-value ${ }^{\dagger}$ & Weighted N & $\%(\mathrm{SE})$ & $P$-value ${ }^{\dagger \dagger}$ \\
\hline \multicolumn{9}{|l|}{ SOCIOECONOMIC } \\
\hline Education & & & & & 0.001 & & & $<0.001$ \\
\hline$<$ High School & 971,041 & $40.2(1.4)$ & 298,300 & $32.9(1.8)$ & & 67,896 & $16.0(1.9)$ & \\
\hline High School/GED & $1,215,968$ & $50.4(1.4)$ & 498,289 & $54.9(2.0)$ & & 232,334 & $54.9(3.2)$ & \\
\hline College Degree & 227,668 & $9.4(0.7)$ & 111,300 & $12.2(1.4)$ & & 123,109 & $29.1(3.2)$ & \\
\hline Income & & & & & $<0.001$ & & & $<0.001$ \\
\hline$<100 \%$ FPL & $1,384,551$ & $56.8(1.2)$ & 428,540 & $46.9(1.7)$ & & 104,719 & $24.7(2.3)$ & \\
\hline $100-<125 \% \mathrm{FPL}$ & 227,591 & $9.3(0.6)$ & 91,940 & $10.1(1.1)$ & & 28,783 & $6.8(1.3)$ & \\
\hline$\geq 125 \%$ FPL & 825,236 & $33.9(1.1)$ & 393,445 & $43.0(1.8)$ & & 290,763 & $68.5(2.6)$ & \\
\hline Employment & & & & & $<0.001$ & & & $<0.001$ \\
\hline Always Unemployed & $1,767,234$ & $72.5(1.2)$ & 496,282 & $54.3(1.8)$ & & 112,490 & $26.5(2.2)$ & \\
\hline Unstable Employment & 301,597 & $12.4(0.8)$ & 228,246 & $25.0(1.6)$ & & 129,583 & $30.6(2.5)$ & \\
\hline Always Employed & 368,547 & $15.1(0.9)$ & 189,398 & $20.7(1.4)$ & & 182,191 & $42.9(2.9)$ & \\
\hline MSA & $1,931,797$ & $79.3(1.8)$ & 721,532 & $78.9(1.9)$ & 0.880 & 344,287 & $81.1(2.7)$ & 0.494 \\
\hline \multicolumn{9}{|l|}{ MEDICAID-RELATED } \\
\hline SSI/TANF & $1,012,240$ & $41.5(1.3)$ & 154,157 & $16.9(1.3)$ & $<0.001$ & 41,212 & $9.7(1.6)$ & $<0.001$ \\
\hline Medicaid HMO/MCO & $1,424,726$ & $61.3(1.5)$ & 459,330 & $50.5(2.0)$ & $<0.001$ & 233,429 & $55.0(3.0)$ & 0.048 \\
\hline
\end{tabular}


Table 3-4. (Continued)

\begin{tabular}{|c|c|c|c|c|c|c|c|c|}
\hline \multirow[b]{2}{*}{ Characteristics } & \multicolumn{2}{|c|}{ Continuous Medicaid } & \multicolumn{3}{|c|}{ Discontinuous-Uninsured } & \multicolumn{3}{|c|}{ Discontinuous-Insured } \\
\hline & Weighted N & $\%(\mathrm{SE})$ & Weighted N & $\%(\mathrm{SE})$ & $P$-value ${ }^{\dagger}$ & Weighted N & $\%(\mathrm{SE})$ & $P$-value ${ }^{\dagger \dagger}$ \\
\hline HEALTH-RELATED & & & & & & & & \\
\hline Perceived Health Status & & & & & 0.162 & & & $<0.001$ \\
\hline Fair to Poor & $1,314,794$ & $53.9(1.2)$ & 467,555 & $51.2(1.9)$ & & 170,598 & $40.2(2.5)$ & \\
\hline Perceived Mental Health & & & & & $<0.001$ & & & $<0.001$ \\
\hline Fair to Poor & 821,318 & $33.7(1.2)$ & 234,931 & $25.7(1.7)$ & & 84,716 & $20.0(2.2)$ & \\
\hline $\begin{array}{l}\text { CVD or high-risk } \\
\text { conditions for CVD }\end{array}$ & & & & & 0.775 & & & 0.037 \\
\hline$\leq 1$ & $1,678,222$ & $68.9(1.1)$ & 640,242 & $70.1(1.8)$ & & 321,685 & $75.8(2.3)$ & \\
\hline 2 & 444,995 & $18.2(0.8)$ & 163,062 & $17.8(1.4)$ & & 65,581 & $15.5(2.2)$ & \\
\hline$\geq 3$ & 314,160 & $12.9(0.8)$ & 110,621 & $12.1(1.3)$ & & 37,000 & $8.7(1.5)$ & \\
\hline $\begin{array}{l}\text { Mental/Substance Abuse } \\
\text { Illnesses }\end{array}$ & 751,357 & $30.8(1.2)$ & 227,885 & $24.9(1.6)$ & 0.003 & 115,891 & $27.3(2.4)$ & 0.176 \\
\hline Respiratory Illnesses & 479,702 & $19.7(1.1)$ & 117,992 & $12.9(1.1)$ & $<0.001$ & 39,380 & $9.3(1.5)$ & $<0.001$ \\
\hline Arthritis/Joint Pain & 830,408 & $34.1(1.3)$ & 267,358 & $29.3(1.7)$ & 0.009 & 107,575 & $25.4(2.4)$ & 0.002 \\
\hline CCI Score Mean (SE) & $1.2(0.0)$ & & $1.1(0.1)$ & & 0.079 & $0.9(0.1)$ & & 0.003 \\
\hline Population Size & $2,437,378$ & $64.6(1.0)$ & 913,926 & $24.2(0.8)$ & & 424,265 & $11.2(0.7)$ & \\
\hline
\end{tabular}




\section{Table 3-4. (Continued)}

Study population includes MEPS respondents from the years 2002-2011, aged 18-64 years, who either reported being diagnosed with or having an event (prescription medication, or inpatient, emergency, outpatient, or office-based provider visits) associated with CVD or highrisk conditions for CVD, had $\leq 1$ month of Medicaid coverage during the survey year.

${ }^{\dagger} P$-value for the difference between Discontinuous-Uninsured and Continuous Medicaid groups. ${ }^{\dagger} P$-value for the difference between

Discontinuous-Insured and Continuous Medicaid groups. $P$-values in bold indicate statistically significant difference below the threshold value of $P<0.05$. SE: Standard error; GED: General educational development; FPL: Federal poverty level; MSA: Metropolitan Statistical Area; SSI: Supplementary Security Income due to disability; TANF: Temporary Assistance to Needy Families program participation; HMO: Health maintenance organization; MCO: Managed care organization; CCI: Charlson Comorbidity Index score (D’Hoore adapted CCI score was calculated in this study using the 3-digit ICD-9-CM codes in MEPS). 
$(P<0.001)$. Among the socioeconomic covariates, this study found that individuals in the Discontinuous-Insured group were more likely to complete high school/GED or college $(P<0.001)$, more likely to have higher family income levels $(P<0.001)$, and were more likely to have some form of employment during the year $(P<0.001)$. The DiscontinuousInsured group was also to be eligible for SSI due to disability or participate in TANF $(P<0.001)$, however, there was no significant difference between having Medicaid through an $\mathrm{HMO} / \mathrm{MCO}(P=0.087)$. Among the health-related covariates, this study found that the Discontinuous-Insured group was less likely to have respiratory illnesses $(P<0.001)$, less likely to report having fair to poor health status $(P<0.001)$, or fair to poor mental health status $(P<0.001)$, and was similar to the Continuous Medicaid coverage group in terms of having mental/substance abuse illnesses $(P=0.428)$, arthritis/joint pain $(P=0.086)$, and mean CCI score or burden of disease $(P=0.078)$. Finally, when examining the medication-related variables, the Discontinuous-Insured group was found to be prescribed lower number of unique medications on average $(P<0.001)$, and more likely to have a 90-day supply of medications $(P<0.001)$ than the Continuous Medicaid group.

For the second aim of examining medication utilization, the patterns in the demographic, socioeconomic, Medicaid -, and health-related characteristics of the study population were found to be similar to the patterns described above, with some exceptions. When examining geographic census region, individuals in the Discontinuous - Uninsured group were found to be more likely to reside in the South, compared to their counterparts in the Continuous Medicaid group. However, the magnitude the statistical significance in difference was small $(P=0.049)$. They were also less likely to have mental/substance abuse illnesses, and arthritis/joint pain. Among the Discontinuous Uninsured group, individuals were less likely to participate in Medicaid administered by an $\mathrm{HMO} / \mathrm{MCO}(P=0.048)$, less likely to have 2 or more $\mathrm{CVD}$ or high-risk conditions for CVD $(P=0.037)$, less likely to have arthritis/joint pain $(P=0.002)$, and had a lower disease burden or mean Charlson score $(P=0.003)$, when compared to those with continuous Medicaid coverage.

\section{Prescription Medication Use in the Study Population}

Table 3-5 displays the summary statistics for prescription medication use in the study population. The distribution of the classes of medications prescribed was similar among all three groups; the Continuous Medicaid, Discontinuous-Uninsured, and the Discontinuous-Insured. Statins and thiazolidinediones were the most commonly prescribed classes of medications, followed by diuretics and anti-hypertensive combination medications. On average, the Continuous Medicaid group reported higher number of all-cause prescription fills annually than the Discontinuous-Uninsured (46.0 vs. 30.0 fills, $P<0.001$ ), and the Discontinuous-Insured groups (46.0 vs. 30.0 fills, $P<0.001)$. Similarly, the Continuous Medicaid group reported higher mean diseasespecific (CVD or high-risk conditions for CVD for CVD) prescription fills annually than the Discontinuous-Uninsured (16.6 vs. 13.5 fills, $P<0.001$ ), and the DiscontinuousInsured groups (16.6 vs. 12.8 fills, $P<0.001)$. The proportion of days during which 
Table 3-5. Summary Statistics for Prescription Medication Use in the Study Population

\begin{tabular}{|c|c|c|c|c|c|c|c|}
\hline \multirow[b]{2}{*}{ Measures } & \multirow[b]{2}{*}{$\begin{array}{l}\text { Therapeutic } \\
\text { Drug Classes }\end{array}$} & \multicolumn{2}{|c|}{ Continuous Medicaid } & \multicolumn{2}{|c|}{ Discontinuous-Uninsured } & \multicolumn{2}{|c|}{ Discontinuous-Insured } \\
\hline & & $\begin{array}{c}\text { Weighted N } \\
\text { or Mean }\end{array}$ & $\begin{array}{r}\%(\mathrm{SE}) \\
\text { or SE }\end{array}$ & $\begin{array}{r}\text { Weighted N } \\
\text { or Mean }\end{array}$ & $\begin{array}{r}\%(\mathrm{SE}) \\
\text { or SE }\end{array}$ & $\begin{array}{l}\text { Weighted N } \\
\text { or Mean }\end{array}$ & $\begin{array}{r}\%(\mathrm{SE}) \\
\text { or SE }\end{array}$ \\
\hline & ACEI/ARB & 136,818 & $6.9(0.7)$ & 47,265 & $6.7(1.0)$ & 28,419 & $8.5(1.8)$ \\
\hline & Beta blockers & 147,001 & $7.4(0.7)$ & 57,759 & $8.1(1.2)$ & 37,979 & $11.4(2.3)$ \\
\hline & $\mathrm{CCB}$ & 125,709 & $6.3(0.6)$ & 57,759 & $6.3(0.9)$ & 13,328 & $4.0(1.1)$ \\
\hline & Diuretics & 288,166 & $14.5(1.0)$ & 105,673 & $14.9(1.5)$ & 39,934 & $11.9(2.2)$ \\
\hline & $\begin{array}{l}\text { Anti-HTN } \\
\text { combinations }\end{array}$ & 197,107 & $9.9(0.7)$ & 72,220 & $10.2(1.0)$ & 42,047 & $12.6(2.0)$ \\
\hline & Statins & 538,014 & $27.1(1.1)$ & 167,148 & $23.6(1.8)$ & 85,635 & $25.6(2.9)$ \\
\hline & Sulfonylureas & 114,938 & $5.9(0.6)$ & 43,755 & $6.2(1.0)$ & 16,097 & $4.8(1.6)$ \\
\hline & Biguanides & 436,045 & $22.0(1.1)$ & 170,483 & $24.0(2.0)$ & 70,987 & $21.2(2.7)$ \\
\hline $\begin{array}{l}\text { Mean No. of Drug } \\
\text { Classes }\end{array}$ & & 2.4 & 0.02 & 2.0 & 0.02 & $1.6^{*}$ & 0.1 \\
\hline $\begin{array}{l}\text { Mean No. of All- } \\
\text { Cause Drug Fills }\end{array}$ & & 46.0 & 1.1 & $30.0^{* * *}$ & 1.3 & $30.0^{* * *}$ & 1.5 \\
\hline $\begin{array}{l}\text { Mean No. of } \\
\text { Disease-Specific } \\
\text { Drug Fills }\end{array}$ & & 16.6 & 0.5 & $13.5^{* * *}$ & 0.6 & $12.8^{* * *}$ & 0.8 \\
\hline
\end{tabular}

Study population included MEPS respondents from the years 2002-2011, aged 18-64 years, who either reported being diagnosed with or having an event (prescription medication, or inpatient, emergency, outpatient, or office-based provider visits) associated with CVD or high-risk conditions for CVD, and who reported having Medicaid anytime during the year.

Estimates in bold indicate statistical significance at $P<0.05$.

ACEI: Angiotensin converting enzyme inhibitors, ARB: Angiotensin II converting enzyme receptor blockers, Calcium channel blockers, HTN:

Hypertension; SE: Standard Error. ${ }^{*} P<0.05, * * P<0.01, * * * P<0.001$ for comparison between the continuous and discontinuous Medicaid groups. 
respondents had possession of medications was significantly higher among the Continuous Medicaid group when compared to the Discontinuous-Uninsured $(67.8 \%$ vs. $64.9 \%, P<0.001)$, but was not significantly different from the Discontinuous-Insured group (67.8\% vs. $68.4 \%, P=0.710)$ (results not shown).

The results for medication adherence outcomes, both overall and therapeutic drugclass-specific, are displayed in Table 3-6. When examining overall adherence using the average MPR measure, this study found that although the Discontinuous-Uninsured group had about $17 \%$ lower odds of being adherent to their medications than the Continuous Medicaid group, this difference was not statistically significant [Odds Ratio (OR): 0.83; 95\% Confidence Interval (CI): 0.69 - 1.00]. Difference in overall adherence was found to be highly insignificant when comparing Discontinuous-Insured and Continuous Medicaid groups $(P=0.775)$. Appendix Table A-1 displays the full model coefficients exponentiated as odds ratios for the comparisons between Continuous Medicaid, Discontinuous-Uninsured, and the Discontinuous-Insured Among the covariates, having fair or poor perceived health status, arthritis/joint pain, and a 90 days' supply of prescription drugs were associated with higher odds of being adherent to commonly prescribed medications that are essential for managing CVD or high-risk conditions for CVD. When examining class-specific medication adherence, this study found that the Discontinuous-Uninsured group were $41 \%$ less likely to be adherent to sulfonylureas when compared to the Continuous Medicaid group (OR: 0.59; 95\% CI: $0.38-0.92$ ). Adherence to the remaining classes of medications examined was not significantly different between these two groups. When examining class-specific adherence among the Discontinuous-Insured, this study found that this group was $43 \%$ less likely to be adherent to diuretics (OR: 0.57; 95\% CI: $0.33-0.98$ ), and had almost 3 times higher odds of being adherent to calcium channel blockers (OR: 2.86; 95\% CI: 1.44 $-5.69)$.

Table 3-7 displays the results for the multivariate negative binomial regression analyses to examine medication utilization. This study found that the incidence of overall prescription fills (all-cause) was 27\% lower among the Discontinuous-Uninsured group compared to the Continuous Medicaid group, or in other words, the expected number of all-cause prescription fills among the Discontinuous-Uninsured group were 27\% less when compared to the Continuous Medicaid group [Incidence Rate Ratio (IRR): 0.73; 95\% CI: $0.68-0.79]$. The average incremental difference in overall prescription medication fills between the Discontinuous-Uninsured and Continuous Medicaid group was found to be -12.0 fills (Standard Error (SE): 1.4 fills; $P<0.001$ ). In other words, individuals in the Discontinuous-Uninsured group were predicted to have 12 fewer prescription drug fills per person annually, compared to the Continuous Medicaid group. Further, it was found that the Discontinuous-Uninsured group had about 12\% lower prescription drug fills annually specific to the CVD or high-risk conditions for CVD for CVD considered in this study (disease-specific), when compared to the Continuous Medicaid group (IRR: 0.88 ; 95\% CI: $0.80-0.97$ ). When estimating the average marginal effect of Medicaid discontinuity, the Discontinuous-Uninsured group was predicted to have about 2.0 fewer disease-specific prescription drug fills per person annually (SE: 0.76 fills; $P=0.009$ ), compared to the Continuous Medicaid group. 
Table 3-6. Association between Medicaid Discontinuity and Prescription Medication Adherence in the Study Population

\begin{tabular}{|c|c|c|}
\hline Outcome & Medicaid Coverage & $\begin{array}{c}\text { Odds Ratio } \\
\text { (95\% Confidence } \\
\text { Interval) } \\
\end{array}$ \\
\hline $\begin{array}{l}\text { Average Adherence } \\
(\text { MPR: }<0.8 \text { v } \geq 0.8)\end{array}$ & $\begin{array}{l}\text { Continuous } \\
\text { Discontinuous-Uninsured } \\
\text { Discontinuous-Insured }\end{array}$ & $\begin{array}{l}1.00 \text { (Reference) } \\
0.83(0.69-1.00) \\
1.04(0.79-1.36)\end{array}$ \\
\hline $\begin{array}{l}\text { Therapeutic Class-Specifi } \\
\text { Adherence (MPR: }<0.8 \mathrm{v} \\
\geq 0.8 \text { ) }\end{array}$ & & \\
\hline$\overline{\mathrm{A}} \mathrm{CEI} / \mathrm{ARB}$ & $\begin{array}{l}\text { Continuous } \\
\text { Discontinuous-Uninsured } \\
\text { Discontinuous-Insured }\end{array}$ & $\begin{array}{l}1.00(\text { Reference }) \\
0.97(0.74-1.28) \\
\mathbf{1 . 5 6}(\mathbf{1 . 0 2}-\mathbf{2 . 3 9}) *\end{array}$ \\
\hline Beta Blockers & $\begin{array}{l}\text { Continuous } \\
\text { Discontinuous-Uninsured } \\
\text { Discontinuous-Insured }\end{array}$ & $\begin{array}{l}1.00 \text { (Reference) } \\
0.74(0.53-1.04) \\
0.84(0.51-1.36)\end{array}$ \\
\hline Calcium Channel Blockers & $\begin{array}{l}\text { Continuous } \\
\text { Discontinuous-Uninsured } \\
\text { Discontinuous-Insured }\end{array}$ & $\begin{array}{l}1.00 \text { (Reference) } \\
1.20(0.86-1.66) \\
\mathbf{3 . 0 6}(\mathbf{1 . 5 6}-\mathbf{6 . 0 1})^{\text {** }}\end{array}$ \\
\hline Diuretics & $\begin{array}{l}\text { Continuous } \\
\text { Discontinuous-Uninsured } \\
\text { Discontinuous-Insured }\end{array}$ & $\begin{array}{l}1.00(\text { Reference }) \\
0.80(0.60-1.08) \\
\mathbf{0 . 5 0}(\mathbf{0 . 2 9}-\mathbf{0 . 8 7}) *\end{array}$ \\
\hline Anti-HTN Combinations & $\begin{array}{l}\text { Continuous } \\
\text { Discontinuous-Uninsured } \\
\text { Discontinuous-Insured }\end{array}$ & $\begin{array}{l}1.00 \text { (Reference) } \\
0.98(0.65-1.50) \\
1.60(0.82-3.15)\end{array}$ \\
\hline Statins & $\begin{array}{l}\text { Continuous } \\
\text { Discontinuous-Uninsured } \\
\text { Discontinuous-Insured }\end{array}$ & $\begin{array}{l}1.00(\text { Reference }) \\
0.85(0.63-1.16) \\
1.02(0.63-1.66)\end{array}$ \\
\hline Sulfonylureas & $\begin{array}{l}\text { Continuous } \\
\text { Discontinuous-Uninsured } \\
\text { Discontinuous-Insured }\end{array}$ & $\begin{array}{l}1.00(\text { Reference }) \\
\mathbf{0 . 6 0}(\mathbf{0 . 3 8}-\mathbf{0 . 9 4}) * \\
0.63(0.28-1.43)\end{array}$ \\
\hline Biguanides & $\begin{array}{l}\text { Continuous } \\
\text { Discontinuous-Uninsured } \\
\text { Discontinuous-Insured }\end{array}$ & $\begin{array}{l}1.00 \text { (Reference) } \\
1.02(0.69-1.50) \\
1.30(0.63-2.67) \\
\end{array}$ \\
\hline
\end{tabular}




\section{Table 3-6. (Continued)}

Study population included MEPS respondents from the years 2002-2011, aged 18-64 years, who either reported being diagnosed with or having an event (prescription medication, or inpatient, emergency, outpatient, or office-based provider visits) associated with CVD or high-risk conditions for CVD, and who reported having Medicaid anytime during the year. Estimates in bold indicate statistical significance at $\mathrm{P}<0.05$. ${ }^{*} P<0.05$, $* * P<0.01, * * * P<0.001$. MPR: Medication Possession Ratio. All models were adjusted for age, gender race/ethnicity, marital status, family size, census region, education, income, employment, MSA, SSI/TANF, Medicaid HMO/MCO, mental/substance abuse illnesses, arthritis/joint pain, no. of CVD conditions, perceived health status, perceived mental health status, Charlson Comorbidity Index score, and survey year. 
Table 3-7. Association between Medicaid Discontinuity and Prescription Medication Utilization in the Study Population

\begin{tabular}{|c|c|c|c|}
\hline Outcome & Medicaid Coverage & $\begin{array}{c}\text { Incidence Rate } \\
\text { Ratio } \\
(\mathbf{9 5 \%} \text { CI })\end{array}$ & $\begin{array}{c}\text { Predicted } \\
\text { Difference in } \\
\text { Events }\end{array}$ \\
\hline \multicolumn{4}{|l|}{ Prescription } \\
\hline \multicolumn{4}{|l|}{ Drug Fills } \\
\hline \multirow[t]{3}{*}{ All-Cause } & Continuous & 1.00 (Reference) & \\
\hline & $\begin{array}{l}\text { Discontinuous- } \\
\text { Uninsured }\end{array}$ & $0.73(0.68-0.79) * * *$ & -12.1 fills**** \\
\hline & Discontinuous-Insured & $0.86(0.76-0.97)^{*}$ & -6.5 fills*** \\
\hline \multirow{3}{*}{$\begin{array}{l}\text { Disease- } \\
\text { Specific }\end{array}$} & Continuous & 1.00 (Reference) & \\
\hline & $\begin{array}{l}\text { Discontinuous- } \\
\text { Uninsured }\end{array}$ & $0.86(0.79-0.95)^{* * *}$ & -2.3 fills** \\
\hline & Discontinuous-Insured & $0.84(0.73-0.96) *$ & -2.8 fills** \\
\hline
\end{tabular}

Study population included MEPS respondents from the years 2002-2011, aged 18-64 years, who either reported being diagnosed with or having an event (prescription medication, or inpatient, emergency, outpatient, or office-based provider visits) associated with CVD or high-risk conditions for CVD, and who reported having Medicaid anytime during the year.

Two separate covariates-adjusted regression models for each outcome comparing Continuous to Discontinuous-Uninsured, and to Discontinuous-Insured, respectively. Estimates in bold indicate statistical significance at $\mathrm{P}<0.05$. MPR: Medication Possession Ratio All models were adjusted for age, gender race/ethnicity, marital status, family size, census region, education, income, employment, MSA, SSI/TANF, Medicaid HMO/MCO, mental/substance abuse illnesses, arthritis/joint pain, no. of CVD conditions, perceived health status, perceived mental health status, Charlson Comorbidity Index score, and survey year.

$* \mathrm{P}<0.05, * * \mathrm{P}<0.01, * * * \mathrm{P}<0.001$ for comparison between the continuous and discontinuous Medicaid groups. 
Medication utilization was similarly lower among the Discontinuous-Insured group. When examining all-cause medication utilization, this study found that the Discontinuous-Insured had approximately 14\% lower prescription drug fills annually (IRR: $0.86 ; 95 \%$ CI: $0.76-0.97$ ), with a predicted marginal difference of 6.5 fewer medication fills per person (SE: $2.4 ; P=0.007$ ), compared to the Continuous Medicaid group. Similarly, the Discontinuous-Insured had an approximately $14 \%$ lower fills of overall prescription medications annually (IRR: 0.86; 95\% CI: $0.74-0.99$ ), and a predicted marginal difference of 2.4 fewer drug fills on average per person annually (SE: $1.1 ; P=0.023$ ), when compared to the Continuous Medicaid coverage population.

\section{DISCUSSION}

Discontinuous Medicaid coverage among adults with CVD or high-risk conditions for CVD was found to have a negative association with prescription drug utilization. The effects were more pronounced among individuals with less than full-year Medicaid coverage and having no other insurance during the year. This DiscontinuousUninsured group was predicted to have 12 fewer fills per person on average for all prescription medications, and about 2 fewer fills per person on average for prescription drugs specific to CVD or high-risk conditions for CVD, compared to the Continuous Medicaid group. Similarly, the Discontinuous-Insured group, or individuals with less than full-year coverage who reported having other sources of insurance coverage during the year, were found to have an average of about 6 fewer drug fills per person overall, and about 3 fewer fills per person for disease-specific medications. Adherence to commonly prescribed medications to manage CVD or high-risk conditions for CVD, measured as average MPR to determine overall concurrent medication adherence, was not found to be significantly different between the continuous and discontinuous Medicaid coverage groups in this study. However, when examining adherence specific to individual therapeutic drug classes, this study found lower adherence among the discontinuous Medicaid groups to sulfonylureas and diuretics. Adherence to ACEI/ARB and calcium channel blockers was found to be higher among the Discontinuous-Insured groups, and was not significantly different between the Discontinuous-Uninsured and the Continuous Medicaid groups as well. Being among the first line therapies to treat high blood pressure that are commonly prescribed, access to these medication classes may not have been impacted by changes in Medicaid coverage during the year in these population groups.

Medication adherence is a highly complex phenomena and barriers to adherence are multifactorial. The World Health Organization (WHO) has done a phenomenal job in classifying the barriers of adherence that capture a wide range of factors that contribute to non-adherence. ${ }^{73}$ As per the WHO classification, barriers to adherence result from: 1) factors associated with the health care team and system in place, 2) disease-related factors, 3) patient-related factors, 4) therapy-related factors, and 5) socio-economic factors. Health system- and health care team-related factors can include lack of access to health care and prescribed medications, and lack of continuity of care due to changes in insurance status, among others. Complex chronic debilitating conditions, such as heart 
failure or stroke, or asymptomatic disorders such as hypertension and diabetes may prevent taking therapies as prescribed or seeking needed medical care. Patient factors such as younger age, minority race or ethnicity, presence of physical or cognitive impairments can also be barriers to medication adherence. Complexity of medication therapy regimen, and side effects of therapies previously undetected in clinical trials can also hinder appropriate management of conditions through pharmacotherapy. ${ }^{74}$ Finally, socioeconomic factors such as low incomes, high medication costs, low literacy, and poor social support may hinder effective use of medications to manage disease conditions. ${ }^{62}$

The present study examined the impact of a health system-related barrier of not having continuous full-year Medicaid coverage on medication adherence after accounting for several other factors that may hinder or confound medication adherence. Given that this study found Medicaid discontinuity to be significantly associated with lower prescription fills, we have strong reasons to believe that having discontinuous Medicaid coverage has a negative impact on prescription medication utilization, and by estimating adherence within a limited time frame of one year using an imputed MPR measure due to absence of complete days' supply information, this study may have underestimated the impact of Medicaid discontinuity on adherence. Adherence to certain classes of antidiabetic and antihypertensive medications was found to be lower among the discontinuous Medicaid groups which further highlights the negative impact that discontinuity and disruptions in Medicaid coverage may have on managing high-risk conditions for CVD.

To our knowledge, there have been no previous studies which have examined the association between discontinuous Medicaid coverage and prescription medication adherence, and the paucity of empirical evidence establishing an association between Medicaid discontinuity and prescription drug utilization, especially among subpopulations with chronic diseases, warranted an in-depth examination of these outcomes. Our findings on prescription drug fills are in line with the findings of Banerjee and colleagues, who examined the impact transitions into and/or out of Medicaid coverage on prescription drug fills, among other outcome measures. ${ }^{15}$ They found that individuals experiencing discontinuity in their Medicaid coverage had lower incidence of prescription drug refills compared to those without any disruptions in their Medicaid coverage. Among studies that investigated disease-specific prescription drug utilization, Smith and Kirking found that individuals with Medicaid coverage disruptions were associated with significantly lower acquisition of medications for HIV/AIDS (human immunodeficiency virus infection and acquired immune deficiency syndrome), such as antiretrovirals. ${ }^{67}$ Fields and colleagues examined the impact of insurance stability and residence on several health care utilization measures and found that discontinuously insured individuals had fewer prescription drug fills, physician office visits, among other outcome measures, compared to those with continuous insurance coverage. ${ }^{32}$ Baicker and colleagues utilized the random assignment of individuals embedded in the Oregon Medicaid lottery to examine the impact of Medicaid coverage on several outcome measures, dubbed the Oregon Experiment. They found that although the use of diabetes medications increased 2 years after the Medicaid lottery to expand Medicaid coverage, the utilization of medications for hypertension or high cholesterol were not significantly 
different. ${ }^{75}$ Differences in sampling, study design (observational vs. random assignment), and duration, besides differences in the study sample may have contributed to the discrepancies between some of these findings and findings from the present study.

Medicaid, the nation's primary public health insurance program for the lowincome population, provides much-needed health insurance coverage to an estimated 67 million individuals who meet the eligibility criteria, which include having incomes and assets below a certain threshold level determined by each State. ${ }^{7}$ Medicaid enrollees have very little copays, with most states requiring about $\$ 1-3$ per drug as copay, although most of them have limits of up to 5 concurrent prescription medications that can be filled per month. ${ }^{76}$ Although seemingly restrictive, studies have found Medicaid enrollees to be more likely to be prescribed medications for certain chronic conditions. Rice and Colleagues in their analysis of the 2001 California Health Interview Survey found that California Medicaid enrollees had higher odds of reporting taking medications for heart disease, high blood pressure, and asthma. ${ }^{77}$ Nonetheless, continuity of insurance coverage, especially for low-income populations, is as critical as gaining insurance coverage, and the findings from this study reinforce the conclusions made by previous studies that discontinuous Medicaid coverage may have an adverse impact on enrollees' prescription drug utilization. ${ }^{15,32,67}$

The literature examining the impact of lack of insurance on health care outcomes in general, and disease-specific outcomes in particular is exhaustive with studies highlighting the adverse impact of lacking health insurance. However, there is a lack of studies examining the impact of discontinuous health insurance on medication outcomes, especially for certain disease conditions such as cardiovascular diseases that require prolonged disease management. And although medication adherence as an area of research has been thoroughly examined with several high-quality robust analyses of adherence and its impact on health care utilization, research examining the impact of churning in insurance in general and Medicaid on medication adherence in particular is limited.

Adherence to medications for CVD or high-risk conditions for CVD varies significantly depending on the study population, and therapeutic classes examined. Studies have shown adherence to several therapeutic classes of medications such as statins, beta blockers, and antiplatelet agents declined following hospitalizations for AMI, $\mathrm{CAD}$, and stroke, and that the trend in decreased adherence continued over periods of more than a year after discharge. ${ }^{66,78-80}$ Using medication event monitoring data, Vrijens and colleagues found medication annual nonadherence rate of 50\% among patients prescribed antihypertensive drugs, whereas Bramley and colleagues in their examination of association between adherence and blood pressure control found $75 \%$ of patients to be adherent to antihypertensives. ${ }^{81,82}$ Bailey and colleagues analyzed Tennessee's Medicaid claims data to examine association between antihypertensive medication adherence and ambulatory visits to hazards of stroke and mortality, and found the mean adherence rate to antihypertensive medications to be $67 \% .{ }^{65}$ Examinations of medication adherence among heath failure patients have also found differing estimates of adherence. ${ }^{83,84}$ Adherence to sulfonylureas was found to be lower among the Discontinuous-Uninsured 
compared to the Continuous Medicaid population. Estimates have found adherence to oral hypoglycemic agents to be low among Medicaid enrollees, and range from 35 to about 50\%. ${ }^{85,86}$ Discontinuous Medicaid coverage with no other coverage during the year may exacerbate the already low levels of adherence to this therapeutic class, which may have been reflected in these findings. Although nonadherence to cardiovascular medications is prevalent, the varying methodologies to estimate adherence, data sources, and study designs, make it difficult to compare results across studies focused on cardiovascular conditions.

This study is not without limitations. Adherence was calculated using an imputed MPR measure that approximated days' supply for medications based on an algorithm created using quantity of medications dispensed. However, unlike previous empirical analyses, this study utilized MEPS data itself to impute MPR, using a plausible assumption that over the 10-year period from 2002, there have not been major changes in the strengths and dosage frequencies of cardiovascular medications. The quality of the prescription drug data in MEPS has been found to be comparable to Medicare claims data, and combined with the high degree of correlation between the imputed and actual days' supply variables, we believe benchmarking our analysis solely to MEPS data is a credible approach. ${ }^{87}$ Additionally, each state establishes their own Medicaid eligibility and procedures for reenrollment, which were not available in MEPS to explore the complex and dynamic patterns of discontinuity that may differ based on the state's eligibility and reporting criteria. Further, this study only explored associations between individual characteristics and Medicaid discontinuity, and not causality, and the findings only explore predictors of within-year discontinuous Medicaid coverage and may not capture risk factors for secular trends in Medicaid discontinuity. Regression models may not have adjusted for certain covariates that may confound the relationship between Medicaid insurance and medication adherence and utilization, or account for unobserved differences between the study population groups. Finally, MEPS data are self-reported, so the estimates may not exactly reflect the national estimates of Medicaid discontinuity. Researchers have speculated that most surveys underestimate the number of Medicaid enrollees due to a variety of reasons including stigma of public insurance. However, such misreporting is less of a problem for MEPS compared to other federal health surveys. ${ }^{54}$ Finally, findings from this study are representative of the non-institutionalized civilian population and may not be applicable to individuals with prolonged hospitalizations or admitted to long-term care facilities.

\section{CONCLUSION}

The findings from this study suggest that continuous Medicaid coverage is critical for non-elderly adults with CVD or high-risk conditions for CVD, and having discontinuous Medicaid coverage was associated with lower prescription drug utilization, and lower adherence to certain therapeutic classes of medications, which are essential components of appropriate disease management. Losing Medicaid coverage may increase the cost sharing burden for former enrollees who have a majority of their maintenance medications covered with nominal copays, resulting in forgoing needed pharmacotherapy, and exacerbating already low levels of adherence to medication 
regimens. Further research with more complete prescription drug data to analyze the impact of Medicaid discontinuity on adherence to important therapeutic classes may better inform policymakers on the determinants of nonadherence, and optimize the delivery of interventions to improve adherence to these vulnerable populations.

Discussions on policies to improve retention of and continuity in Medicaid must include individuals suffering from these chronic and debilitating conditions whose pharmacotherapy may be adversely impacted due to such coverage instability. 


\section{CHAPTER 4. ASSOCIATIONS BETWEEN MEDICAID DISCONTINUITY, ACCESS TO CARE, PREVENTIVE CARE, AND HEALTH SERVICES UTILIZATION AMONG ADULTS WITH CARDIOVASCULAR DISEASE OR ASSOCIATED HIGH-RISK CONDITIONS}

\section{BACKGROUND}

Medicaid provides much-needed health insurance coverage to an estimated 67-70 million low-income and disabled children, adults, and elderly at any given point in time, who meet the eligibility criteria, which include having incomes and assets below a certain threshold level determined by each State. ${ }^{7,88}$ Recent estimates indicate that over $28 \%$ of non-elderly adult Medicaid enrollees with family incomes at or below 138 percent of the federal poverty level (FPL) are diagnosed with cardiovascular disease (CVD), including heart disease and stroke. ${ }^{20}$ Conditions such as hypertension, hypercholesterolemia, and diabetes, among others, are significant risk factors for CVD; however, these conditions are preventable and manageable. For low-income vulnerable populations having CVD or high-risk conditions for CVD, accessing needed medical care, prescription drugs, routine preventive care, and periodic primary care visits are extremely critical to manage their complex chronic conditions. Failure to do so may exacerbate their cardiovascular symptoms leading to prolonged morbidity and result in avoidable hospitalizations that may exert a substantial financial stress on the health care system.

Overwhelming evidence suggests that access to medical care, routine preventive services, and having a usual source of care for primary care visits are critical in managing disease conditions, and maintaining good health. ${ }^{89-92}$ Having stable, continuous health insurance coverage has been shown to be critical in ensuring that individuals do not have to forego medical care or have high financial burden, ensure appropriate preventive care services are accessed on a timely basis, and have regular access to primary care providers to obtain the medical care necessary for preventing and treating their chronic diseases. ${ }^{2,93-}$

${ }^{95}$ Poor disease management, resulting from inadequate access to care, lack of adherence to pharmacotherapy, or lack of sufficient access to primary care physicians may result in increased risk of hospitalizations that could have been avoided. ${ }^{91,96-98}$

Low family incomes, assessed as a percentage of the federal poverty level, family status, residence, and assets test, are some of the criteria used to determine eligibility for Medicaid coverage, which are specific to each states. Although most states have moved towards a 12 month coverage renewal for non-elderly adults in recent years, they may still require certain individuals to periodically report their income and/or other criteria in order to maintain eligibility. Upward income mobility would render individuals ineligible for their state's Medicaid coverage if their incomes cross the eligibility threshold; however, research has shown that individuals may passively end up not reenrolling into Medicaid due to a variety of reasons, ranging from lack of awareness to various barriers faced in reenrollment. ${ }^{99}$ This results in individuals and families losing Medicaid coverage within 12 months of enrollment. Instability in Medicaid, characterized by dropouts and moving back and forth into various sources of coverage or a state of being uninsured, has 
been a long-standing issue that has impacted non-elderly adult Medicaid enrollees due to stringent administrative regulations governing coverage for this population. ${ }^{9,100}$ Previous estimates have shown that about $43 \%$ of individuals who are newly enrolled in Medicaid, end up losing their Medicaid coverage within twelve months. ${ }^{38}$ Transitions into and out of Medicaid coverage may adversely impact the ability for low-income populations with CVD or high-risk conditions for CVD to seek, and maintain continuity in, medical care. Losing Medicaid coverage to become uninsured would be detrimental, as there is strong consensus regarding the negative outcomes associated with lack of insurance. ${ }^{10-12,31,32}$ However, transitioning between Medicaid and other sources of insurance coverage may also prove to be problematic, as this may lead to moving between different provider networks, and coverage with different benefit structures that may limit use of previously covered health care resources.

Estimates of the impact of discontinuous Medicaid coverage on access to care and utilization of health care services among individuals with CVD or high-risk conditions for CVD are very few and dated. Findings from state-specific Medicaid data have found Medicaid coverage discontinuity to be associated with higher inpatient and ER hospitalizations associated, especially for individuals with heart disease, diabetes, among other ambulatory care sensitive conditions. ${ }^{14,101}$ Newer, more comprehensive national estimates of the impact of discontinuous Medicaid coverage on access to care, preventive services use, and health care services utilization among individuals with CVD or its risk factor chronic conditions are critical to determine the outcomes among vulnerable diseased populations for whom insurance coverage continuity may be even more critical. The present study aims to obtain national estimates among adults with CVD or high-risk conditions for CVD of associations between Medicaid discontinuity and (1) access to care, (2) preventive services use, and (3) utilization of overall (all-cause) and diseasespecific health care services, such as hospital inpatient visits, emergency room (ER) visits, hospital outpatient visits, and physician office visits. We hypothesized that having discontinuous Medicaid coverage, relative to full-year continuous Medicaid coverage, would be associated with significantly poor access to care, lower preventive services use, lower primary care utilization, and higher hospitalizations in this study population.

\section{METHODS}

The present study is a retrospective cross-sectional analysis comparing adult populations with CVD or high-risk conditions for CVD, with and without continuous full-year Medicaid coverage. Instability and disruptions in Medicaid coverage may especially have a detrimental impact on the continuity of care among low-income adults with CVD or any of its risk factor conditions. We hypothesized that Medicaid discontinuity would be associated with poor access to care, decreased primary care characterized by lower preventive services use and physician visits - and increased hospitalizations, both overall (all-cause), and those specific to CVD or high-risk conditions for CVD (disease-specific). 


\section{Data Source}

The present study analyzed data from the Medical Expenditure Panel Survey (MEPS). MEPS is a set of large-scale surveys of families, individuals, their medical providers and employers across the United States. It is jointly sponsored by the Agency for Healthcare Research and Quality (AHRQ) and the National Center for Health Statistics (NCHS), and has been conducted annually since 1996. It has two major components; the Household Component (HC), the Insurance Component, with data on medical providers in the HC being supplemented by the Medical Provider Component. MEPS collects detailed information from a nationally representative sample of the civilian noninstitutionalized population of the U.S. on health services utilization and health expenditures, insurance coverage, and sources of payment.

MEPS has an overlapping panel design. Two separate panels of respondents are interviewed simultaneously during a calendar year. A new panel of sample households is selected each year, and data for each panel are collected for two calendar years. The two years of data for each panel are collected in five rounds of interviews that take place over a two and a half year period. This provides continuous and current estimates of health care expenditures at both the person and household level for two panels for each calendar year. By combining data from the overlapping panels, these annual files provide nearly double the sample size of individual panels and cover the entire calendar year for each respondent. Although all MEPS data are reported by respondents during computerassisted personal interviewing, further detailed health service use data, including on prescription drugs, are collected from a sample of providers with respondents' permission. ${ }^{39}$

When obtaining national estimates from surveys such as MEPS, appropriate sample size is critical to obtain reliable estimates, since estimates of some population subgroups may vary from year to year. The advantage of survey such as MEPS is that they allow for several years' of data to be pooled together in order to increase the sample size and improve the precision of estimates. The pooling of the different years of data was conducted in accordance with the guidelines provided by MEPS, and data for the years $2002-2011$ were pooled together to allow for a sufficiently large sample size to ensure reliable estimates.

\section{Study Population}

The broad classification of "cardiovascular disease (CVD) and associated comorbidities" in this study consisted for the following disease conditions: acute myocardial infarction (Clinical Classification code (CCC): 100), coronary artery disease (CCC: 101), congestive heart failure (CCC: 108), peripheral and visceral atherosclerosis (CCCs: 114, 115, 116), stroke (CCCs: 109, 112), hypertension (CCC: 098), lipid disorders (CCC: 053), and diabetes (CCC: 049, 050). CCC aggregates conditions and procedures into mutually exclusive and clinically homogeneous categories, using Clinical Classification Software. ${ }^{40}$ The study population included respondents who were: (1) aged 
18 to 64 years, (2) reported either being diagnosed with CVD or high-risk conditions for CVD for CVD in their first round of the MEPS survey, or reported having an event (an inpatient, emergency, outpatient, or office-based provider visits, or a prescription medication) associated with one or more of the aforementioned disease conditions at any point of time during the survey, (3) reported having had Medicaid coverage at any point of time during the survey year, and (4) had positive person weights, and were interviewed for all five rounds of the 2-year MEPS survey interviews. Respondents who reported having Medicare, or pregnancy were excluded from this study.

Respondents who reported having Medicare, or pregnancy were excluded from this study. Pregnant women with incomes at or below 133 percent of the federal poverty level are eligible for Medicaid coverage throughout the pregnancy; however, the coverage ceases 60 days postpartum. ${ }^{68}$ Low-income non-elderly adults with disabilities are also eligible for Medicare if they have received monthly social security disability income for two years. Most coverage benefits for prescription drugs for dual eligible beneficiaries are covered by Medicare Part D, instead of Medicaid. As a result, these two population groups were excluded from this study.

Individuals with less than a year of Medicaid coverage who experience withinyear Medicaid discontinuity may not be a homogenous group, especially when they report having other forms of insurance coverage during the year. In order to account for these differences, individuals in the study population who reported having $<12$ months of Medicaid coverage during a survey year were divided into two groups: (1) individuals with $<12$ months of Medicaid coverage who did not report any other sources of health insurance during the survey year, designated in this study as 'Discontinuous-Uninsured', and (2) individuals with $<12$ months of Medicaid coverage who also reported having other source(s) of health insurance during the survey year (either private, Tricare, or any hospital- or physician group-based health insurance), designated in this study as 'Discontinuous-Insured'. Individuals in the study population who reported having Medicaid coverage during all 12 months of a survey year were classified as having continuous Medicaid coverage, which was the comparison group and designated as 'Continuous Medicaid' in this study.

\section{Study Variables}

Our first objective was to examine whether Medicaid discontinuity was associated with poor access to care among non-elderly adults with CVD or high-risk conditions for CVD. The outcomes of interest for this objective were the following three binary indicators for: (1) having a usual source of care, (2) having difficulty in obtaining medical care, and (3) having difficulty obtaining prescription medications. When examining association between Medicaid discontinuity and preventive services use, the following three binary outcomes were examined: (1) having blood pressure checkup within the past year, (2) having cholesterol checkup within the past year, and (3) having routine medical checkup within the past year. For the final objective of examining associations between Medicaid discontinuity, primary care visits, and hospitalizations, we 
divided the utilization outcome measures into all-cause, i.e. overall utilization during the survey year irrespective of diagnosis, and disease-specific utilization, i.e. utilization specific to CVD or high-risk conditions for CVD considered in this study. Specifically, the total number of inpatient visits, ER visits, hospital outpatient visits, and physician office visits, were examined, resulting in eight outcome measures (one all-cause, and one disease-specific for each) that were characterized as discrete counts of the events

All regression models included the following classes of covariates:-

1) Demographic covariates: age (in years), gender (female vs. male), race/ethnicity (Non-Hispanic White vs. Non-Hispanic Black, Hispanic, and other races), marital status (not married vs. married), family size $(\mathrm{N})$, and geographic residence (Northeast vs. Midwest, South, and West).

2) Socioeconomic covariates: education (less than high school vs. high school/GED, and Bachelor's or higher), income as a percentage of the federal poverty level (FPL) $(<100 \%$ FPL vs. $100-<125 \%$ FPL, and $\geq 125 \%$ FPL), employment (always unemployed vs. intermittent employment, and always employed), binary indicator for Metropolitan Statistical Area (MSA) residence.

3) Medicaid-related covariates: binary indicator for Supplementary Security Income (SSI) for disability receipt or participation in the Temporary Assistance for Needy Families program, and a binary indicator for Medicaid HMO/Managed Care participation.

4) Health-related covariates: perceived health status (excellent/very good/good vs. fair/poor), perceived mental health status (excellent/very good/good vs. fair/poor) binary indicator for mental illness/substance abuse diagnosis, binary indicator for respiratory diseases diagnosis, binary indicator for arthritis/joint pain diagnosis, and Charlson Comorbidity Index (CCI) score.

Additionally, all of the multivariate models included the year fixed effects to capture any macro-level variations during the study period.

\section{Statistical Analysis}

Bivariate statistics were conducted to examine the characteristics of the study population. When examining the binary outcomes for access to care and preventive services use, adjusted multivariate logistic regression analyses were conducted. As an example, Equation 4-1 and Equation 4-2 depict the logistic regression models usual source of care, as part of the access to care measures, among both the DiscontinuousUninsured, and the Discontinuous-Insured groups relative to the Continuous Medicaid insurance group.

$$
\begin{aligned}
& \operatorname{Pr}[\mathrm{USC}=1 \mid \mathrm{x}]=\mathrm{F}^{-1}\left(\hat{\alpha}+\hat{\beta}_{1} \cdot \text { Discontinuous }- \text { Uninsured }+\sum_{k=1}^{K} \hat{\beta}_{k} X_{k}\right) \\
& \operatorname{Pr}[\mathrm{USC}=1 \mid \mathrm{x}]=\mathrm{F}^{-1}\left(\hat{\alpha}+\hat{\beta}_{1} . \text { Discontinuous }- \text { Insured }+\sum_{k=1}^{K} \hat{\beta}_{k} X_{k}\right)
\end{aligned}
$$


Usual source of care is modeled as a dichotomous outcome variable as a function of Medicaid discontinuity as well as the demographic, socioeconomic, Medicaid-related, and health-related covariates mentioned above. $\mathrm{F}^{-1}$ denotes the inverse of the cumulative standard logistic distribution function that relates the outcome on the probability scale to the covariates. $\operatorname{Pr}[$ ] denotes the population average probability of having a usual source of care (denoted by 1) that is conditional on the covariates denoted by x. DiscontinuousUninsured and Discontinuous-Insured are binary indicator variables for the main independent variable of interest, that equals one for individuals having discontinuous annual Medicaid coverage without any other sources of insurance and with other sources of insurance during the year, respectively, and equals zero for the comparison group of individuals with continuous Medicaid coverage. $X$ denotes the vector of the aforementioned individual characteristics that predict Medicaid discontinuity in the two population groups of interest.

The number of physician office visits data (both all-cause and disease-specific) displayed overdispersion, however, this count outcome measure did not have excess zeros. Thus, an adjusted negative binomial regression was utilized. It is a type of generalized linear model where the dependent variable $Y$ is a count of the number of times an event occurs (in this case, the number of physician office visits). A convenient parameterization of the negative binomial distribution is denoted by Equation 4-3. ${ }^{71}$

$$
p(y)=P(Y=y)=\frac{\Gamma\left(y+\frac{1}{\alpha}\right)}{\Gamma(y+1) \Gamma\left(\frac{1}{\alpha}\right)}\left(\frac{1}{1+\alpha \mu}\right)^{1 / \alpha}\left(\frac{\alpha \mu}{1+\alpha \mu}\right)^{y}
$$

In Equation 4-3, $\mu>0$ is the mean of $Y$, which is the count variable indicating the number of prescription drug fills (either all-cause, or disease-specific) and $\alpha>0$ is the heterogeneity parameter. It can be derived as a Poisson-gamma mixture, or as the number of failures before the $(1 / \alpha)^{\text {th }}$ success. A simpler depiction of the traditional negative binomial regression model, the NB2 model is depicted is depicted in Equation 4-4 and Equation 4-5 as follows,

$$
\begin{aligned}
& \ln \mu_{\text {all-cause }}=\beta_{0}+\beta_{1} \text { Discontinuous }- \text { Uninsured }+\beta_{2} \cdot x_{2}+\cdots+\beta_{k} \cdot x_{k} \text { (Eq. 4-4) } \\
& \ln \mu_{\text {disease-specific }}=\beta_{0}+\beta_{1} \text { Discontinuous }- \text { Uninsured }+\beta_{2} \cdot x_{2}+\cdots+\beta_{k} \cdot x_{k}
\end{aligned}
$$

where, apart from the main indicator variables for the Discontinuous-Uninsured group, the covariates $x_{2}$ to $x_{k}$ are known, and the population regression coefficients $\beta_{0}$ to $\beta_{k}$ are to be estimated. The two equations above depict the negative binomial regression models to estimate the differences in overall (Equation 4-4), and disease-specific physician office visits (Equation 4-5) between the Continuous Medicaid, and Discontinuous-Uninsured groups. Similar regression models (not shown here) were run to estimate differences in the number of physician office visits between the Continuous Medicaid and Discontinuous-Insured groups. 
When analyzing the discrete count data for the number of all-cause and diseasespecific inpatient visits, ER visits, and hospital outpatient visits, it was important to account for the overdispersion in the data characterized by the presence of an excessive number of zeros, a problem that is commonly known as zero inflation (ZI). Appendix B, Figures B-1 to B-16, display the distribution of the all-cause and disease-specific outcome variables examined in this study. The distribution of the ZI data is often rightskewed, with a huge peak at zero, representing the individuals with zero health care consumption, and a skewed tail representing the rest of the study population with varying amounts of non-zero consumption. ZI data are often analyzed using a two-part mixture models combining a point mass at zero with a proper count distribution. These models assume an initial process to determine membership into one of two latent groups, generally referred to the 'susceptible' and 'non-susceptible' population groups. ${ }^{102}$ The approach for ZI models uses two regression models, one is usually a logit regression (or probit) modeling the susceptible probability, and the other is either a Poisson or negative binomial model, modeling the mean for the susceptible population (Equation 4-6). Explanatory variables are allowed to have a different impact for the two processes, and the two models are fit simultaneously using maximum likelihood estimation. ${ }^{102}$ The outcomes measures with excess zeros were therefore analyzed using zero inflated negative binomial regression models, mathematically represented as follows:

$$
P(Y=y)\left\{\begin{array}{cl}
p+(1-p)\left(\frac{1}{1+\alpha \mu}\right)^{\frac{1}{\alpha}}, & y=0 \\
(1-p) \frac{\Gamma\left(y+\frac{1}{\alpha}\right)}{\Gamma(y+1) \Gamma\left(\frac{1}{\alpha}\right)}\left(\frac{1}{1+\alpha \mu}\right)^{\frac{1}{\alpha}}\left(\frac{\alpha \mu}{1+\alpha \mu}\right)^{y}, & y=1,2, \ldots
\end{array}\right.
$$

Having a mean $E(Y)=(1-p) \mu$, and a variance $\operatorname{Var}(Y)=(1-p) \mu(1+p \mu+\alpha \mu)$. Research has shown zero-inflated negative binomial models to provide superior fit when compared to zero-inflated Poisson regression. ${ }^{103}$ For the binary model estimating the susceptible probability, the following variables were included

The coefficient of interest for the Discontinuous-Uninsured is the measure of differences in the utilization of overall, and disease-specific drug utilization. Because of the functional form of the negative binomial regression, the effect size of a factor is the antilog of its coefficient. This estimated coefficients are transformed to obtain the incidence rate ratio (IRR). An IRR of less than one for the discontinuous Medicaid groups would suggest lower hospital outpatient utilization for instance, among them when compared to their counterparts with continuous Medicaid coverage.

In addition to obtaining IRRs, this study further estimated the average marginal effect to determine the predicted difference in the number of all-cause and diseasespecific prescription drug fills between the continuous and discontinuous Medicaid groups. The marginal effects measure the discrete change in the outcome variable, i.e. for a binary independent variable, how do predicted probabilities change as the binary independent variable changes from 0 (Continuous Medicaid) to 1 (DiscontinuousUninsured, or Discontinuous-Insured). ${ }^{72}$ In order to obtain these marginal effects, after developing and running the multivariate negative binomial regression model, the 
estimated coefficients of the model were used to calculate two predicted event visits for all individuals in the study population. The first predicted utilization assumed all individuals had discontinuous Medicaid coverage (by setting the Medicaid discontinuity indicator variable to 1), and the second predicted utilization assumed all individual to have continuous full-year Medicaid coverage (by setting the Medicaid discontinuity indicator variable to 0 ). The average per-person difference in the event visits attributable to Medicaid coverage was estimated by obtaining the difference in predicted drug utilization for each person and computing the weighted average of the difference across the entire study sample.

The proportion of missing data was around $6 \%$ in this study, as a result, the listwise deletion approach was used to account for missing values. Multicollinearity was assessed for all the regression models, and the variance inflation factor was found to be less than 5, which was lower than the widely accepted threshold of 10 for existence of multicollinearity. The Hosmer-Lemeshow tests for goodness-of-fit were highly insignificant for all the logistic regression models indicating that the model fit the data. All data analyses were conducted using SAS 9.3 and STATA 13 to account for the complex survey design of MEPS.

\section{RESULTS}

\section{Characteristics of the Study Populations}

Table 4-1 displays the characteristics of the study population. The study sample included 3,926 respondents in the Continuous Medicaid coverage group (nationally representative of about 2.4 million individuals), 1,407 individuals in the DiscontinuousUninsured group (nationally representative of over 900,000 individuals), and 493 individuals in the Discontinuous-Insured group (nationally representative of over 400,000 individuals).

When compared to the Continuous Medicaid group, individuals in the Discontinuous-Uninsured group were less likely to belong to other race groups $(P<0.001)$, more likely to be married $(P<0.001)$, and were more likely to reside in the South region $(P=0.049)$. Among the socioeconomic covariates, this study found that respondents were more likely to have completed high school/GED or college $(P=0.001)$, were more likely to have family incomes $\geq 125 \%$ FPL $(P<0.001)$, and more likely to have intermittent, as well as continuous full-year employment $(P<0.001)$. Respondents were less likely to be eligible for SSI due to disability or participate in TANF $(P<0.001)$, and less likely to report being Medicaid managed care enrollees $(P<0.001)$. Among the health-related variables, respondents were less likely to report having fair or poor perceived mental health status $(P<0.001)$, less likely to report having mental/substance abuse illnesses $(P=0.003)$, less likely to report having respiratory illnesses $(P<0.001)$, and less likely to report having arthritis/joint pain $(P=0.009)$. The mean CCI score was not significantly different between the two groups $(P=0.181)$. 
Table 4-1. Characteristics of the Study Population (2002 - 2011)

\begin{tabular}{|c|c|c|c|c|c|c|c|c|}
\hline \multirow[b]{2}{*}{ Characteristics } & \multicolumn{2}{|c|}{ Continuous Medicaid } & \multicolumn{3}{|c|}{ Discontinuous-Uninsured } & \multicolumn{3}{|c|}{ Discontinuous-Insured } \\
\hline & Weighted N & $\%(\mathrm{SE})$ & Weighted N & $\%(\mathrm{SE})$ & $P$-value ${ }^{\dagger}$ & Weighted N & $\%(\mathrm{SE})$ & $P$-value ${ }^{\dagger \dagger}$ \\
\hline DEMOGRAPHIC & & & & & & & & \\
\hline Age & & & & & 0.385 & & & 0.013 \\
\hline $18-34$ years & 388,425 & $15.9(1.0)$ & 165,340 & $18.1(1.3)$ & & 98,188 & $23.1(2.3)$ & \\
\hline $35-49$ years & 875,809 & $35.9(1.2)$ & 324,653 & $35.5(1.8)$ & & 141,109 & $33.3(2.7)$ & \\
\hline $50-64$ years & $1,173,144$ & $48.2(1.3)$ & 423,933 & $46.4(2.0)$ & & 184,968 & $43.6(3.0)$ & \\
\hline Gender & & & & & 0.491 & & & 0.169 \\
\hline Female & $1,632,006$ & $67.0(1.2)$ & 624,410 & $68.3(1.8)$ & & 267,502 & $63.1(2.8)$ & \\
\hline Race/Ethnicity & & & & & $<0.001$ & & & $<0.001$ \\
\hline Non-Hispanic White & $1,094,002$ & $44.9(1.8)$ & 442,534 & $48.4(2.1)$ & & 256,231 & $60.4(3.2)$ & \\
\hline Non-Hispanic Black & 666,358 & $27.3(1.5)$ & 219,033 & $24.0(1.6)$ & & 79,936 & $18.8(2.0)$ & \\
\hline Hispanic & 445,601 & $18.3(1.2)$ & 202,106 & $22.1(1.5)$ & & 50,704 & $12.0(1.6)$ & \\
\hline Others & 231,416 & $9.5(0.9)$ & 50,253 & $5.5(0.9)$ & & 37,393 & $8.8(1.7)$ & \\
\hline Marital Status & & & & & $<0.001$ & & & $<0.001$ \\
\hline Married & 727,457 & $29.9(1.3)$ & 344,150 & $37.7(1.8)$ & & 184,414 & $43.5(3.2)$ & \\
\hline Region & & & & & 0.049 & & & 0.023 \\
\hline Northeast & 608,070 & $25.0(1.6)$ & 187,785 & $20.5(1.7)$ & & 104,394 & $24.6(2.9)$ & \\
\hline Midwest & 477,401 & $19.6(1.4)$ & 184,710 & $20.2(1.7)$ & & 115,146 & $27.2(3.2)$ & \\
\hline South & 727,520 & $29.8(1.6)$ & 315,073 & $34.5(1.8)$ & & 98,072 & $23.1(2.3)$ & \\
\hline West & 624,386 & $25.6(1.7)$ & 226,358 & $24.8(1.9)$ & & 106,652 & $25.1(3.0)$ & \\
\hline Family Size Mean (SE) & $2.8(0.1)$ & & $2.9(0.1)$ & & 0.409 & $2.8(0.1)$ & & 0.976 \\
\hline
\end{tabular}


Table 4-1. (Continued)

\begin{tabular}{|c|c|c|c|c|c|c|c|c|}
\hline \multirow[b]{2}{*}{ Characteristics } & \multicolumn{2}{|c|}{ Continuous Medicaid } & \multicolumn{3}{|c|}{ Discontinuous-Uninsured } & \multicolumn{3}{|c|}{ Discontinuous-Insured } \\
\hline & $\begin{array}{r}\text { Weighted } \\
\mathbf{N}\end{array}$ & $\%(\mathrm{SE})$ & Weighted N & $\%(\mathrm{SE})$ & $P$-value ${ }^{\dagger}$ & Weighted N & $\%(\mathrm{SE})$ & $P$-value ${ }^{\dagger \dagger}$ \\
\hline \multicolumn{9}{|l|}{ SOCIOECONOMIC } \\
\hline Education & & & & & 0.001 & & & $<0.001$ \\
\hline$<$ High School & 971,041 & $40.2(1.4)$ & 298,300 & $32.9(1.8)$ & & 67,896 & $16.0(1.9)$ & \\
\hline High School/GED & $1,215,968$ & $50.4(1.4)$ & 498,289 & $54.9(2.0)$ & & 232,334 & $54.9(3.2)$ & \\
\hline College Degree & 227,668 & $9.4(0.7)$ & 111,300 & $12.2(1.4)$ & & 123,109 & $29.1(3.2)$ & \\
\hline Income & & & & & $<0.001$ & & & $<0.001$ \\
\hline$<100 \%$ FPL & $1,384,551$ & $56.8(1.2)$ & 428,540 & $46.9(1.7)$ & & 104,719 & $24.7(2.3)$ & \\
\hline $100-<125 \% \mathrm{FPL}$ & 227,591 & $9.3(0.6)$ & 91,940 & $10.1(1.1)$ & & 28,783 & $6.8(1.3)$ & \\
\hline$\geq 125 \% \mathrm{FPL}$ & 825,236 & $33.9(1.1)$ & 393,445 & $43.0(1.8)$ & & 290,763 & $68.5(2.6)$ & \\
\hline Employment & & & & & $<0.001$ & & & $<0.001$ \\
\hline Always Unemployed & $1,767,234$ & $72.5(1.2)$ & 496,282 & $54.3(1.8)$ & & 112,490 & $26.5(2.2)$ & \\
\hline Unstable Employment & 301,597 & $12.4(0.8)$ & 228,246 & $25.0(1.6)$ & & 129,583 & $30.6(2.5)$ & \\
\hline Always Employed & 368,547 & $15.1(0.9)$ & 189,398 & $20.7(1.4)$ & & 182,191 & $42.9(2.9)$ & \\
\hline MSA & $1,931,797$ & $79.3(1.8)$ & 721,532 & $78.9(1.9)$ & 0.880 & 344,287 & $81.1(2.7)$ & 0.494 \\
\hline \multicolumn{9}{|l|}{ MEDICAID-RELATED } \\
\hline SSI/TANF & $1,012,240$ & $41.5(1.3)$ & 154,157 & $16.9(1.3)$ & $<0.001$ & 41,212 & $9.7(1.6)$ & $<0.001$ \\
\hline Medicaid HMO/MCO & $1,424,726$ & $61.3(1.5)$ & 459,330 & $50.5(2.0)$ & $<0.001$ & 233,429 & $55.0(3.0)$ & 0.048 \\
\hline
\end{tabular}


Table 4-1. (Continued)

\begin{tabular}{|c|c|c|c|c|c|c|c|c|}
\hline \multirow[b]{2}{*}{ Characteristics } & \multicolumn{2}{|c|}{ Continuous Medicaid } & \multicolumn{3}{|c|}{ Discontinuous-Uninsured } & \multicolumn{3}{|c|}{ Discontinuous-Insured } \\
\hline & Weighted N & $\%(\mathrm{SE})$ & Weighted N & $\%(\mathrm{SE})$ & $P$-value ${ }^{\dagger}$ & Weighted N & $\%(\mathrm{SE})$ & $P$-value ${ }^{\dagger \dagger}$ \\
\hline HEALTH-RELATED & & & & & & & & \\
\hline Perceived Health Status & & & & & 0.162 & & & $<0.001$ \\
\hline Fair to Poor & $1,314,794$ & $53.9(1.2)$ & 467,555 & $51.2(1.9)$ & & 170,598 & $40.2(2.5)$ & \\
\hline Perceived Mental Health & & & & & $<0.001$ & & & $<0.001$ \\
\hline Fair to Poor & 821,318 & $33.7(1.2)$ & 234,931 & $25.7(1.7)$ & & 84,716 & $20.0(2.2)$ & \\
\hline $\begin{array}{l}\text { CVD or high-risk } \\
\text { conditions for CVD }\end{array}$ & & & & & 0.775 & & & 0.037 \\
\hline$\leq 1$ & $1,678,222$ & $68.9(1.1)$ & 640,242 & $70.1(1.8)$ & & 321,685 & $75.8(2.3)$ & \\
\hline 2 & 444,995 & $18.2(0.8)$ & 163,062 & $17.8(1.4)$ & & 65,581 & $15.5(2.2)$ & \\
\hline$\geq 3$ & 314,160 & $12.9(0.8)$ & 110,621 & $12.1(1.3)$ & & 37,000 & $8.7(1.5)$ & \\
\hline $\begin{array}{l}\text { Mental/Substance Abuse } \\
\text { Illnesses }\end{array}$ & 751,357 & $30.8(1.2)$ & 227,885 & $24.9(1.6)$ & 0.003 & 115,891 & $27.3(2.4)$ & 0.176 \\
\hline Respiratory Illnesses & 479,702 & $19.7(1.1)$ & 117,992 & $12.9(1.1)$ & $<0.001$ & 39,380 & $9.3(1.5)$ & $<0.001$ \\
\hline Arthritis/Joint Pain & 830,408 & $34.1(1.3)$ & 267,358 & $29.3(1.7)$ & 0.009 & 107,575 & $25.4(2.4)$ & 0.002 \\
\hline CCI Score Mean (SE) & $1.2(0.0)$ & & $1.1(0.1)$ & & 0.079 & $0.9(0.1)$ & & 0.003 \\
\hline Population Size & $2,437,378$ & $64.6(1.0)$ & 913,926 & $24.2(0.8)$ & & 424,265 & $11.2(0.7)$ & \\
\hline
\end{tabular}

Study population includes MEPS respondents from the years 2002-2011, aged 18-64 years, who either reported being diagnosed with or having an event (prescription medication, or inpatient, emergency, outpatient, or office-based provider visits) associated with CVD or high-risk conditions for CVD, had $\leq 1$ month of Medicaid coverage during the survey year. ${ }^{\dagger} P$-value for the difference between Discontinuous-Uninsured and Continuous Medicaid groups. ${ }^{\dagger} P$-value for the difference between Discontinuous-Insured and Continuous Medicaid groups. $P$-values in bold indicate statistically significant difference at $P<0.05$. SE: Standard error; GED: General educational development; FPL: Federal poverty level; MSA: Metropolitan Statistical Area; SSI: Supplementary Security Income. 
When comparing the Discontinuous-Insured group to the Continuous Medicaid group in the above table, this study found that among the demographic covariates, individuals in the Discontinuous-Insured group were more likely to be younger $(P=0.013)$, were less likely to belong to minority race/ethnicity groups $(P<0.001)$, more likely to be married $(P<0.001)$, and were more likely to reside in the Midwest $(P=0.023)$. Among the socioeconomic covariates, this study found that individuals in the Discontinuous-Insured group were more likely to complete high school/GED or college $(P<0.001)$, more likely to have higher family income levels $(P<0.001)$, and were more likely to have some form of employment during the year $(P<0.001)$. The DiscontinuousInsured group was also less likely to be eligible for SSI due to disability or participate in TANF $(P<0.001)$, and less likely to report having Medicaid through an HMO/MCO $(P=0.048)$. Among the health-related covariates, this study found that the DiscontinuousInsured group was less likely to report having self-perceived health status $(P<0.001)$, and self-perceived mental health status $(P<0.001)$, more likely to report fewer CVD or highrisk conditions for $\mathrm{CVD}(P=0.037)$, were less likely to report having respiratory illnesses $(P<0.001)$, less likely to report having arthritis/joint pain $(P=0.002)$, and lower chronic disease burden $(P=0.003)$.

\section{Medicaid Enrollment among the Discontinuous Medicaid Coverage Groups}

Table 4-2 shows the summary statistics for Medicaid enrollment among the discontinuous Medicaid insurance groups. The average duration of enrollment for the undivided discontinuous Medicaid population was 6.0 months. The DiscontinuousUninsured group was enrolled in Medicaid for a longer period on average compared to the Discontinuous-Insured group (6.5 vs. 5.0 months, $P<0.001)$. Additionally, this study examined the average duration of no insurance and found individuals in the Discontinuous-Insured group were uninsured for a little over a month (5.5 vs. 1.2 months, $P<0.001)$. This study also examined Medicaid enrollment status after 12 months of initial Medicaid enrollment. Overall, a total of 4,898 individuals were enrolled in the month of January of the survey year, of which $85.3 \%$ were still enrolled in Medicaid during the month of December in the survey year, 3.5\% had some other form of insurance coverage, and 10.2\% were uninsured (results not shown). Of the 621 individuals in the Discontinuous-Uninsured group who had Medicaid in the beginning of the year, $78.1 \%$ were uninsured while $21.9 \%$ still had Medicaid coverage at the end of the year. Of the 200 individuals in the Discontinuous-Insured group who had Medicaid in the beginning of the survey year, only $8.0 \%$ still had Medicaid, $6.0 \%$ were uninsured, and the remaining $86 \%$ had some other source of health insurance coverage at the end of the year. Finally, when examining the number of transitions into and/or out of Medicaid coverage, this study found that overall $17.6 \%$ of individuals in this study population had more than one transitions, and the proportion of multiple transitions was similar between the two discontinuous Medicaid subgroups (17.7\% vs. $17.3 \%, P=0.875)$. 
Table 4-2. Patterns of Medicaid Coverage among Discontinuous Medicaid Groups

\begin{tabular}{|c|c|c|c|c|}
\hline \multirow[b]{2}{*}{$\begin{array}{l}\text { Coverage } \\
\text { Components }\end{array}$} & \multicolumn{2}{|c|}{ Discontinuous-Uninsured } & \multicolumn{2}{|c|}{ Discontinuous-Insured } \\
\hline & $\begin{array}{c}\text { Mean or } \\
\text { Weighted N }\end{array}$ & $\begin{array}{l}\text { SE or \% } \\
\text { (SE) }\end{array}$ & $\begin{array}{c}\text { Mean or } \\
\text { Weighted N }\end{array}$ & $\begin{array}{l}\text { SE or \% } \\
\text { (SE) }\end{array}$ \\
\hline $\begin{array}{l}\text { Mean Duration of } \\
\text { Medicaid }\end{array}$ & 6.5 months & 0.1 & 5.0 months $^{* * *}$ & 0.2 \\
\hline $\begin{array}{l}\text { Mean Duration of No } \\
\text { Insurance }\end{array}$ & 5.5 months & 0.1 & 1.2 months $^{* * *}$ & 0.1 \\
\hline $\begin{array}{l}\text { Insurance Status } \\
\text { After } 12 \text { Months }\end{array}$ & & & & \\
\hline Still in Medicaid & 83,125 & $21.9(2.2)$ & $11,647^{* * *}$ & $8.0(2.0)$ \\
\hline Uninsured & 297,045 & $78.1(2.2)$ & $10,058^{* * *}$ & $6.0(2.0)$ \\
\hline Other Insurance & -- & -- & 147,896 & $86.0(2.0)$ \\
\hline $\begin{array}{l}\text { Medicaid Coverage } \\
\text { Transitions }\end{array}$ & & & & \\
\hline 1 & 752,311 & $82.3(1.4)$ & 350,803 & $82.7(2.0)$ \\
\hline$\geq 2$ & 161,615 & $17.7(1.4)$ & 73,462 & $17.3(2.0)$ \\
\hline
\end{tabular}

Sample consisted of 380,171 individuals in the Discontinuous-Uninsured group and 169,600 individuals in the Discontinuous-Insured group who either reported being diagnosed with or having an event (prescription medication, or inpatient, emergency, hospital outpatient, or office-based provider visits) associated with CVD or high-risk conditions for CVD, and who reported having Medicaid at the beginning of the year.

$* * * P<0.001 ; * * P<0.01 ; * P<0.05$ for difference from the Discontinuous-Uninsured group. 


\section{Access to Care}

In comparison to the Continuous Medicaid group, individuals in the Discontinuous-Uninsured group were less likely to report having a usual source of care (93.6\% vs. $84.5 \% ; P<0.001)$, and more likely to report having difficulties in obtaining medical care $(5.6 \%$ vs. $19.1 \% ; P<0.001)$, and prescription medications $(5.8 \%$ vs. $15.8 \%$; $P<0.001)$. When compared to the Continuous Medicaid group, those in the Discontinuous-Insured group were less likely to report having a usual source of care (93.6\% vs. $88.6 \% ; P=0.002)$, and more likely to report having difficulty in obtaining prescription medications $(5.8 \%$ vs. $11.3 \% ; P<0.001)$; however, there were no differences between the two groups in facing difficulty in obtaining medical care $(P=0.094)$. Results of these bivariate analyses are not displayed. Table 4-3 displays the results of the multivariate logistic regression examining association between Medicaid discontinuity and access to care, among adults with CVD or high-risk conditions for CVD. In comparison to the Continuous Medicaid group, the Discontinuous-Uninsured group were found to have almost $60 \%$ lower odds of having a usual source of care [Odds Ratio (OR): 0.41; 95\% Confidence Interval (CI): 0.32 - 0.52]. Additionally, the odds were a staggering 4 times higher of reporting having difficulty in obtaining in obtaining medical care (OR: 4.20; 95\% CI: 3.14 - 5.62), and were more than 3 times higher of reporting having difficulty in obtaining prescription medications (OR: 3.32; 95\% CI: $2.50-4.40$ ) when compared to those with continuous Medicaid coverage. Among the DiscontinuousInsured group, the odds of having a usual source of care were $43 \%$ lower when compared to the Continuous Medicaid group (0.57; 95\% CI: $0.37-0.88)$. The odds of reporting having difficulty in obtaining medical care were 74\% higher (OR: 1.74; 95\% CI: 1.02 2.98), and the odds of reporting having difficulty in obtaining prescription medications were more than twice, when compared to the Continuous Medicaid group (OR: 2.16; $95 \%$ CI: $1.42-3.27)$.

\section{Preventive Services Use}

When examining the bivariate statistics between the Continuous Medicaid and Discontinuous-Uninsured groups, this study found no significant differences between the two when reporting about the routine BP checkup $(P=0.073)$ and routine cholesterol checkup $(P=0.140)$; however, the Discontinuous-Uninsured group was less likely to report having had a medical checkup within the past year $(P=0.004)$. When comparing the Continuous Medicaid and Discontinuous-Insured groups, this study found that those in the Discontinuous-Insured group were less likely to report having routine BP checkup (96.9\% vs. $94.5 \% ; P=0.023)$, whereas differences in routine cholesterol checkup $(P=$ $0.897)$ and medical checkup $(P=0.146)$ were insignificant (results not displayed). Table 4-4 displays the results of the multivariate logistic regression examining association between Medicaid discontinuity and preventive services use, among adults with CVD or high-risk conditions for CVD. When examining routine blood pressure checkup and routine blood cholesterol checkup, there were no significant differences between the continuous and discontinuous Medicaid populations. However, the DiscontinuousUninsured were found to have $22 \%$ lower odds of having had a medical checkup within 
Table 4-3. Association between Medicaid Discontinuity and Access to Care in the Study Population

\begin{tabular}{|c|c|c|}
\hline Outcome & Medicaid Coverage & $\begin{array}{c}\text { Odds Ratio } \\
\text { (95\% Confidence Interval) }\end{array}$ \\
\hline \multirow[t]{3}{*}{ Have Usual Source of Care } & Continuous & 1.00 (Reference) \\
\hline & Discontinuous-Uninsured & $0.41(0.32-0.52) * * *$ \\
\hline & Discontinuous-Insured & $0.57(0.37-0.88)^{*}$ \\
\hline Difficulty in Obtaining & Continuous & 1.00 (Reference) \\
\hline \multirow[t]{2}{*}{ Necessary Medical Care } & Discontinuous-Uninsured & $4.20(3.14-5.62) * * *$ \\
\hline & Discontinuous-Insured & $1.74(1.02-2.98)^{*}$ \\
\hline Difficulty in Obtaining & Continuous & 1.00 (Reference) \\
\hline \multirow[t]{2}{*}{ Prescription Medications } & Discontinuous-Uninsured & $3.32(2.50-4.40) * * *$ \\
\hline & Discontinuous-Insured & $2.16(1.42-3.27) * * *$ \\
\hline
\end{tabular}

Study population included MEPS respondents from the years 2002-2011, aged 18-64 years, who either reported being diagnosed with or having an event (prescription medication, or inpatient, emergency, outpatient, or office-based provider visits) associated with CVD or high-risk conditions for CVD, and who reported having Medicaid anytime during the year.

Two separate covariates-adjusted regression models for each outcome comparing Continuous to Discontinuous-Uninsured, and to Discontinuous-Insured, respectively. Estimates in bold indicate statistical significance at $\mathrm{P}<0.05 .{ }^{*} P<0.05,{ }^{*} P<0.01, * * * P<0.001$ for comparison between the continuous and discontinuous Medicaid groups.

All models were adjusted for age, gender race/ethnicity, marital status, family size, census region, education, income, employment, MSA, SSI/TANF, Medicaid HMO/MCO, mental/substance abuse illnesses, arthritis/joint pain, no. of CVD conditions, perceived health status, perceived mental health status, Charlson Comorbidity Index score, and survey year. 
Table 4-4. Associations between Medicaid Discontinuity and Preventive Services Use in the Study Population

\begin{tabular}{llc}
\hline \multicolumn{1}{c}{ Outcome } & \multicolumn{1}{c}{ Medicaid Coverage } & $\begin{array}{c}\text { Odds Ratio } \\
\text { (95\% Confidence Interval) }\end{array}$ \\
\hline Routine BP Checkup & Continuous & 1.00 (Reference) \\
& $\begin{array}{l}\text { Discontinuous-Uninsured } \\
\text { Discontinuous-Insured }\end{array}$ & $0.90(0.61-1.31)$ \\
& & $0.75(0.42-1.33)$ \\
Routine Cholesterol Checkup & Continuous & 1.00 (Reference) \\
& Discontinuous-Uninsured & $0.85(0.64-1.13)$ \\
& Discontinuous-Insured & $1.06(0.68-1.64)$ \\
Routine Medical Checkup & Continuous & $1.00($ Reference) \\
& Discontinuous-Uninsured & $\mathbf{0 . 7 8}(\mathbf{0 . 6 3}-\mathbf{0 . 9 6})^{*}$ \\
& Discontinuous-Insured & $0.80(0.56-1.13)$ \\
& & \\
\hline
\end{tabular}

Study population included MEPS respondents from the years 2002-2011, aged 18-64 years, who either reported being diagnosed with or having an event (prescription medication, or inpatient, emergency, outpatient, or office-based provider visits) associated with CVD or high-risk conditions for CVD, and who reported having Medicaid anytime during the year.

Two separate covariates-adjusted regression models for each outcome comparing Continuous to Discontinuous-Uninsured, and to Discontinuous-Insured, respectively. Estimates in bold indicate statistical significance at $\mathrm{P}<0.05 .{ }^{*} P<0.05,{ }^{*} P<0.01, * * * P<0.001$ for comparison between the continuous and discontinuous Medicaid groups.

All models were adjusted for age, gender race/ethnicity, marital status, family size, census region, education, income, employment, MSA, SSI/TANF, Medicaid HMO/MCO, mental/substance abuse illnesses, arthritis/joint pain, no. of CVD conditions, perceived health status, perceived mental health status, Charlson Comorbidity Index score, and survey year. 
the past year when compared to the Continuous Medicaid population (OR: $0.78 ; 95 \% \mathrm{CI}$ : $0.63-0.96)$.

\section{Utilization of Health Care Services}

The results of the final objective of examining associations between Medicaid discontinuity and heath care services utilization are displayed in Table 4-5. Overall inpatient visits were not significantly different between the continuous and discontinuous Medicaid groups. However, when examining disease-specific inpatient visits, this study found that the Discontinuous-Uninsured group had about $62 \%$ higher inpatient visits compared to the Continuous Medicaid group [Incidence Rate Ratio (IRR): 1.62; 95\% CI: $1.19-2.22]$. The marginal predicted difference in the number of inpatient visits was found to be 0.05 visits $(P=0.007)$; in other words, individuals in the DiscontinuousUninsured group were predicted to have 0.05 higher inpatient visits per person annually, compared to the Continuous Medicaid group. Similarly, the incidence of disease-specific inpatient visits was found to be $95 \%$ higher among the Discontinuous-Insured population (IRR: $1.95 ; 95 \%$ CI: $1.23-3.10$ ), which at the margins was predicted to be 0.08 higher inpatient visits per person $(P=0.029)$ when compared to the Continuous Medicaid group. When examining ER visits, this study did not find significant associations between Medicaid discontinuity and all-cause ER visits. When examining disease-specific ER visits, this study found that the incidence of these visits were $52 \%$ higher among the Discontinuous-Uninsured population, and the predicted marginal difference from the Continuous Medicaid group was found to be 0.05 additional visits per person $(P=0.008)$. The Discontinuous-Insured too had a 79\% higher incidence of disease-specific ER visits compared to the Continuous Medicaid population, with a predicted difference of 0.07 greater ER visits per person $(P=0.034)$.

Among the primary care utilization outcomes, this study found the Discontinuous-Uninsured group to have an $86 \%$ higher incidence of disease-specific hospital outpatient visits compared to the Continuous Medicaid insurance group (IRR: $1.86 ; 95 \%$ CI: $1.32-2.63$ ), and a predicted marginal difference of 0.21 additional visits $(P=0.007)$. Finally, the Discontinuous-Uninsured group was found to have approximately $29 \%$ lower incidence of all-cause physician office visits (IRR: $0.71 ; 95 \%$ CI: $0.64-0.80$ ), and were predicted to have 3.4 fewer overall physician office visits per person annually, when compared to their counterparts with continuous Medicaid coverage $(P<0.001)$.

\section{DISCUSSION}

Adults with CVD or high-risk conditions for CVD who did not have continuous full-year Medicaid coverage, due to either loss of coverage, or disruptions/gaps, reported significantly worse health care and medication access, and routine healthcare checkup visits. Additionally, those in with discontinuous Medicaid coverage reported lower 
Table 4-5. Associations between Medicaid Discontinuity and Health Care Resources Utilization in the Study Population

\begin{tabular}{|c|c|c|c|}
\hline Outcome & Medicaid Coverage & $\begin{array}{c}\text { Incidence Rate Ratio } \\
\text { (95\% Confidence Interval) }\end{array}$ & $\begin{array}{c}\text { Predicted } \\
\text { Difference in Visits }\end{array}$ \\
\hline \multicolumn{4}{|l|}{ Inpatient Visits } \\
\hline \multirow[t]{3}{*}{ All-Cause } & Continuous & 1.00 (Reference) & \\
\hline & Discontinuous-Uninsured & $1.14(0.95-1.37)$ & 0.05 visits \\
\hline & Discontinuous-Insured & $1.24(0.97-1.58)$ & 0.08 visits \\
\hline \multirow[t]{3}{*}{ Disease-Specific } & Continuous & 1.00 (Reference) & \\
\hline & Discontinuous-Uninsured & $1.62(1.19-2.22) * *$ & 0.05 visits** \\
\hline & Discontinuous-Insured & $1.95(1.23-3.10) * *$ & 0.08 visits* \\
\hline \multicolumn{4}{|c|}{ Emergency Room Visits } \\
\hline \multirow[t]{3}{*}{ All-Cause } & Continuous & 1.00 (Reference) & \\
\hline & Discontinuous-Uninsured & $0.96(0.79-1.17)$ & -0.02 visits \\
\hline & Discontinuous-Insured & $1.00(0.73-1.38)$ & 0.00 visits \\
\hline \multirow[t]{3}{*}{ Disease-Specific } & Continuous & 1.00 (Reference) & \\
\hline & Discontinuous-Uninsured & $1.52(1.14-2.03) * *$ & 0.05 visits** \\
\hline & Discontinuous-Insured & $1.79(1.16-2.77) * *$ & 0.07 visits* \\
\hline \multicolumn{4}{|c|}{ Hospital Outpatient Visits } \\
\hline \multirow[t]{3}{*}{ All-Cause } & Continuous & 1.00 (Reference) & \\
\hline & Discontinuous-Uninsured & $0.77(0.55-1.06)$ & -0.33 visits \\
\hline & Discontinuous-Insured & $1.20(0.71-2.04)$ & 0.28 visits \\
\hline \multirow[t]{3}{*}{ Disease-Specific } & Continuous & 1.00 (Reference) & \\
\hline & Discontinuous-Uninsured & $1.86(1.32-2.63) * * *$ & 0.21 visits $* *$ \\
\hline & Discontinuous-Insured & $0.86(0.47-1.60)$ & -0.04 visits \\
\hline
\end{tabular}


Table 4-5. (Continued)

\begin{tabular}{llcc}
\hline \multicolumn{1}{c}{ Outcome } & \multicolumn{1}{c}{ Medicaid Coverage } & $\begin{array}{c}\text { Incidence Rate Ratio } \\
\mathbf{9 5 \%} \text { Confidence Interval) }\end{array}$ & $\begin{array}{c}\text { Predicted } \\
\text { Difference in Visits }\end{array}$ \\
\hline $\begin{array}{l}\text { Physician Office Visits } \\
\text { All-Cause }\end{array}$ & Continuous & 1.00 (Reference) \\
& Discontinuous-Uninsured & $\mathbf{0 . 7 1 ( \mathbf { 0 . 6 2 } - \mathbf { 0 . 8 1 } ) * *}$ & $0.89(0.77-1.11)$ \\
& Discontinuous-Insured & $1.00($ Reference) & -1.27 visits \\
Disease-Specific & Continuous & $0.90(0.80-1.02)^{*}$ & -0.30 visits \\
& Discontinuous-Uninsured & $0.86(0.71-1.06)$ & -0.43 visits \\
\hline
\end{tabular}

Study population included MEPS respondents from the years 2002-2011, aged 18-64 years, who either reported being diagnosed with or having an event (prescription medication, or inpatient, emergency, outpatient, or office-based provider visits) associated with CVD or high-risk conditions for CVD, and who reported having Medicaid anytime during the year. Estimates in bold indicate statistical significance at $\mathrm{P}<0.05$.

All models were adjusted for age, gender race/ethnicity, marital status, family size, census region, education, income, employment, MSA, SSI/TANF, Medicaid HMO/MCO, mental/substance abuse illnesses, arthritis/joint pain, no. of CVD conditions, perceived health status, perceived mental health status, Charlson Comorbidity Index score, and survey year. $* P<0.05, * * P<0.01, * * * P<0.001$ for comparison between the continuous and discontinuous Medicaid groups 
physician office visits, and higher inpatient, and ER hospitalizations. In examining these associations, this study further classified individuals with discontinuous Medicaid coverage into those who did not report any other coverage (the DiscontinuousUninsured), and those who reported having other sources of insurance coverage during the year (the Discontinuous-Insured), in order to capture the heterogeneity of the discontinuous Medicaid group. In doing so, we found that the outcomes of Medicaid discontinuity were not always similar, either in magnitude, directionality, or statistical significance. For instance, in comparison to the Continuous Medicaid group, among both the Discontinuous-Uninsured and Discontinuous-Insured groups, the access to care outcomes were significantly poor; however, the odds of reporting difficulty in obtaining medical care were over 4 times, and almost 2 times higher, respectively. Similarly, the Discontinuous-Uninsured population in this study had significantly lower odds of reporting routine medical checkup within the past year, whereas no significant difference in this outcome was observed between the Discontinuous-Insured and the Continuous Medicaid populations.

Nearly three-quarters $(74.1 \%)$ of the individuals who did not have continuous full-year Medicaid coverage in this CVD population belonged to the DiscontinuousUninsured group. On average, this group was uninsured for over 5 months during the year, and around 18\% of individuals experienced multiple transitions in their Medicaid coverage, indicating significant churning, and relapses into the state of being uninsured. Of the roughly 400,000 individuals who had Medicaid at the beginning of the year, a whopping $78 \%$ were found to be uninsured by the end of the year. These individuals collectively had worse outcomes associated with Medicaid discontinuity when compared to those who reported other sources of insurance coverage during the year, the Discontinuous-Insured, with significantly fewer physician office visits and higher incidences of inpatient and ER hospitalizations, when compared to those with continuous Medicaid coverage. Although preventive services use, and primary care visit outcomes were not significantly poor among the Discontinuous-Insured, these individuals did collectively have poor access to medical care and pharmacotherapy, and higher incidences of hospitalizations when compared to those with continuous Medicaid coverage. Our study findings indicate that for individuals with CVD or some of its risk factor conditions that require continuous, prolonged disease management, lacking continuous Medicaid coverage may adversely impact access to care, and primary care, and have a strong association with increased incidences of hospitalizations, irrespective of whether or not they have other sources of coverage during the year. And these outcomes may worsen among individuals and families for whom Medicaid is the only source of health insurance coverage.

The increased hospitalizations, both inpatient and ER, were The results of this study, are in line with previous estimates, however, very few have examined Medicaid discontinuity outcomes among adults with CVD or high-risk conditions for CVD. Among the earliest studies, Lurie and colleagues examined access to care among a cohort of California Medicaid enrollees within a 6-month period after loss of coverage. A significantly higher proportion of individuals in the lost coverage group (62\%) reported poor access to needed medical care, compared to those still on Medicaid (7\%), with the 
discontinuous Medicaid group being more likely to have uncontrolled hypertension. ${ }^{104}$ In a follow-up study conducted at one year found no improvements in access to care between the discontinuous and continuous Medicaid groups. ${ }^{105}$ Carlson and colleagues examined the impact of disrupted or lost Medicaid coverage within a year of benefit structure changes among the Oregon Medicaid program, on unmet medical care or pharmacotherapy needs, utilization, and medical debt. ${ }^{106}$ They found that enrollees with discontinuous Medicaid coverage were more likely to report unmet medical care, prescription medication needs (lost Medicaid coverage group), and were less likely to report having primary care visits, when compared to those enrolled continuously in the program.

Harman and colleagues examined associations between interruptions in a state Medicaid program coverage and inpatient psychiatric services among persons with schizophrenia. ${ }^{10}$ They found an $86 \%$ increase in the incidence of hospitalizations, representing 0.63 more psychiatric hospitalizations per beneficiary, and longer hospital stays to be associated with discontinuous Medicaid coverage. Hall and colleagues examined the impact of gaps in Florida's Medicaid coverage on health care utilization and expenditures among individuals with diabetes. ${ }^{101}$ That study found that gaps in coverage were associated with higher inpatient and ER utilization, and higher expenditures, especially in the period after lapse in Medicaid coverage. Banerjee and colleagues analyzed the MEPS data for the years 2000 - 2004 to examine association between Medicaid discontinuity and health resource utilization among all Medicaid enrollees. ${ }^{15}$ They defined discontinuity as having one or more than one transitions into or out of Medicaid coverage, which was different from the present study. The aforementioned study found Medicaid discontinuity to be associated with higher inpatient, ER, and higher outpatient physician visits. It is unclear whether they examined hospital outpatient visits and physician office visits separately, or combined the two outcomes together. Nonetheless, the results from this study are broadly in agreement with the study of Banerjee and colleagues, indicating that Medicaid discontinuity, either due to coverage loss or disruptions in coverage continuity have detrimental outcomes, both overall, and among individuals with chronic conditions. Alternatively, it may be possible that the results obtained in the analyses of Medicaid enrollees in general, may in particular be contributed by individuals with chronic, debilitating conditions, such as CVD or conditions that are high risk factors for CVD, as this study has shown.

Medicaid provides much-needed health care coverage to the poor and disabled vulnerable populations who often are unable to afford private health insurance. Medicaid is often referred to as a "Cadillac" health insurance program, which provides a rich benefits package at very little or even no cost to the enrollees. ${ }^{107}$ A substantial body of research has shown that low-income Medicaid enrollees have comparable access to physician care, preventive services, and unmet medical care needs when compared to their counterparts with private health insurance. ${ }^{107}$ Even low-income mothers on Medicaid report similar preventive services use and access to care compared to those with private insurance. ${ }^{108}$ Together, these and other estimates shown the importance of Medicaid coverage for low-income populations, and dispel the notion that Medicaid is a costly, low-quality health coverage program. ${ }^{109,110}$ Loss of Medicaid coverage may 
deprive these vulnerable populations suffering from chronic conditions of the ability to afford and seek much-needed medical care and pharmacotherapy. Such disruptions in continuity of care may be responsible for respondents reporting lack of usual source of care and difficulties in obtaining medical care and prescription drugs. The Discontinuous-Uninsured group also reported lower incidence of physician office visits overall, which may be precipitated by the lack of usual source of care, and inability of afford physician visit reimbursements following loss of coverage. This group reported higher rates of hospital outpatient visits compared to those with continuous Medicaid coverage, a finding that was puzzling. It may be that these individuals may be more inclined to seek care in the outpatient setting following loss of Medicaid coverage, and subsequent inability to afford physician office visits as highlighted earlier. The higher incidences of disease-specific hospitalizations observed in this study could have been precipitated by poor access to care, lower likelihood of getting routine medical checkups, lower primary care office visits, and the disruptions in continuity of care resulting from loss of Medicaid coverage. A follow-up evaluation of the Oregon Health Experiment, found higher ER utilization among individuals who gained Medicaid coverage, relative to those who remained uninsured. ${ }^{111}$ The present study found increases in CVD or high-risk conditions for CVD-specific ER visits among the discontinuous Medicaid groups compared to the Continuous Medicaid group, which may have been precipitated by poor access to care as observed in our findings, and also due to disruptions in disease management caused by loss or gaps in Medicaid coverage. Differences in the study population (diseased vs. general population, and national vs. state-specific) and estimation techniques may have contributed to the differences in our findings from the Oregon analysis of ER utilization.

This study has several limitations. State-level Medicaid eligibility data was not available to explore the complex and dynamic patterns of discontinuity that may differ based on the state's eligibility and reporting criteria. This study found strong associations between Medicaid discontinuity and poor access to care, lower odds of routine medical checkup, higher disease-specific hospitalizations, and lower all-cause physician office visits. Nonetheless, temporality between discontinuity and the various outcomes, and the resulting causality could not be established due to data limitations. The regression analyses may not have adjusted for unobserved covariates that may have confounded the impact of certain risk factors on the likelihood of belonging to the DiscontinuousUninsured or the Discontinuous-Insured groups. Data in MEPS are self-reported, and the reasons for discontinuity of Medicaid coverage were not captured, resulting in estimates that may not exactly reflect the national estimates of the impact of Medicaid discontinuity on access to care, preventive services use, and health care services utilization.

Researchers have speculated that most surveys underestimate the number of Medicaid enrollees due to a variety of reasons including stigma of public insurance. However, such misreporting is less of a problem for MEPS compared to other federal health surveys. ${ }^{54}$ Finally, findings from this study are representative of the non-institutionalized civilian population and may not be applicable to individuals with prolonged hospitalizations or admitted to long-term care facilities. 
Loss of Medicaid coverage has an adverse impact on individuals' ability to seek medical care which may in turn lead to poor health outcomes and increased burden on the health care system resulting from providing care to uninsured individuals. Individuals eligible for Medicaid may also lose coverage due to the inefficient administrative hurdles and burdensome paperwork requirements, and may be considered a policy failure. ${ }^{34}$ However, when individuals transition from one source of coverage to another, continuity of care may still be impacted, especially since different insurance plans have different network of providers, and differing benefit structures, and may cover different classes of medications and services. The present study highlighted the adverse impact that Medicaid discontinuity may have on ability to access needed medical care, and the increased hospitalizations for CVD or high-risk conditions for CVD associated with coverage instability. Enabling continuity in Medicaid coverage for low-income populations with chronic and debilitating conditions would ensure increased effectiveness of the care they receive to manage these disease, and may prove cost-effective in the long run.

Discontinuity in Medicaid and the Children's Health Insurance Program (CHIP) coverage among low-income children is an issue that has gained considerable scrutiny previously, and the detrimental effects of such a policy failure have been well established. ${ }^{35,112}$ Apart from simplification of administrative regulations governing policy renewals, a policy initiative adopted by most states is the 12 months continuous eligibility provision. Under this policy, children continue to be enrolled under Medicaid/CHIP irrespective for 12 months after gaining or renewing coverage, irrespective of any income or other eligibility midway. Researchers and advocacy groups, such as the Medicaid and CHIP Payment Access Commission, have advocated for extending such a measure for non-elderly adults to ensure continuity of Medicaid coverage in this population. ${ }^{8,44}$ Our study findings indicate that individuals with chronic conditions requiring prolonged and continuous disease management, such as CVD or its high-risk conditions, may be especially adversely impacted by instability in their Medicaid coverage. States can choose to have a straight-forward option of establishing a minimum guaranteed eligibility period, such as the 12 month continuous eligibility provision, for subpopulations of nonelderly adults with chronic conditions, such as CVD, or other such as cancers, respiratory illnesses, mental illnesses, HIV/AIDS, among others, which require prolonged management of disease symptoms and risk factors. By using annual eligibility redetermination periods, and guaranteeing enrollment for the period in between, states can ensure that loss of coverage or churning midway during enrollment can be reduced in these vulnerable populations.

\section{CONCLUSION}

The present study highlighted the significantly worse access to care, preventive medical checkups, lower primary care office visits, and higher hospitalizations for CVD or high-risk conditions for CVD, associated with having discontinuous Medicaid coverage. Policy initiatives to stabilize Medicaid coverage among non-elderly adults may provide the most benefit for individuals with chronic conditions requiring continuous long-term medical care interventions, and appropriate disease management. Low-income 
adults managing chronic conditions, especially in regions with stringent Medicaid eligibility regulations, must be encouraged to ensure continuity of Medicaid coverage. States may adopt annual eligibility reassessment policies, with continuous eligibility for the intermittent period, for non-elderly adults with certain chronic conditions among whom continuous Medicaid coverage would ensure greater effectiveness of disease management. 


\section{CHAPTER 5. CONCLUSION}

\section{SUMMARY}

The present study was aimed at conducting a detailed examination of the impact of having discontinuous Medicaid coverage on various health care utilization outcomes and medication consumption behaviors, which are extremely crucial in effectively preventing and treating chronic diseases of the cardiovascular system along with many of its risk factor disease conditions. Although previous estimates have characterized general populations that experience discontinuous Medicaid coverage, and compared them with their counterparts with continuous Medicaid insurance, none have focused on national estimates of subpopulations diagnosed with complex chronic conditions requiring prolonged disease management, such as CVD or chronic conditions which are high risk factors for CVD. The first aim of this dissertation intended to characterize the populations of non-elderly adults with CVD or high-risk conditions for CVD who have discontinuous Medicaid coverage, and compare them to their counterparts with continuous Medicaid coverage. Another difference between this analyses and previous ones is the classification of the subpopulations with less than full-year Medicaid coverage. Individuals with discontinuous Medicaid coverage are not a homogenous population, since although many individuals may not have any other source of insurance coverage during the year, the Discontinuous-Uninsured group in this study, this population would also comprise of individuals with other sources of coverage, ranging from private insurance, Tricare, or other hospital-, or physician group-based health insurance. Interestingly, this study found about three-fourths of this population to belong to the Discontinuous-Uninsured group, and a little over a fourth to belong to the Discontinuous-Insured group.

Hypertension, lipid disorders, and diabetes, significant risk factors for CVD, were found to be the most prevalent chronic conditions in this study population. These are diseases that require prolonged, and sometimes lifelong treatment, which includes adequate preventive care to monitor health or disease progression, getting regular medical checkups and making regular primary care visits to diagnose and treat these conditions early, and filling prescription medications regularly and consuming them as prescribed by health care providers. This finding, coupled with the fact that insurance is a major source that enables individuals, especially low-income sick populations, to seek needed medical care and pharmacotherapy, further strengthens our justification for examining the impact of Medicaid discontinuity among low-income populations with CVD or high-risk conditions for CVD.

When examining Medicaid coverage patterns among the two discontinuous Medicaid coverage groups, this study found that on average, the DiscontinuousUninsured were uninsured for over 5 months during the year, and the DiscontinuousInsured too were uninsured on average for a little over a month, despite reporting other insurance sources. Further, a subsample of individuals who had coverage at the beginning of the year were examined to determine their coverage patterns by the year end. It was observed that among the Discontinuous-Uninsured who had Medicaid coverage at the 
start of the year, $78 \%$ were uninsured by the year end. Among the DiscontinuousInsured, only $8 \%$ had Medicaid, $86 \%$ had other insurance, and around $6 \%$ were uninsured by the end of the year. Finally, this study found that almost $18 \%$ of both the discontinuous Medicaid groups had more than one transitions, indicating a decent amount of churning taking place in these groups, i.e. going back-and-forth from Medicaid to either a state of being uninsured, or enrolling in another source of insurance coverage, or vice versa (uninsured - Medicaid - uninsured, or other insurance - Medicaid - other insurance). These findings highlight the instability in Medicaid coverage encountered by low-income, chronically diseased, non-elderly adult population, and the disruptions in continuity of care associated with transitioning from Medicaid to other sources of coverage, or vice versa, not to mention the difficulties involved with adjusting to differences in provider networks, services covered, services and resources covered, and benefit structures.

When examining the population characteristics associated with Medicaid discontinuity among the Discontinuous-Uninsured relative to the Continuous Medicaid group, this study found that those who were married, resided in the South, had intermittent unstable employment, and had fair to poor perceived health status, had higher odds of having discontinuous Medicaid coverage, whereas men, minorities, those with higher incomes, SSI/TANF beneficiaries, Medicaid managed care enrollees, and those with respiratory illnesses had lower odds of experiencing discontinuity in their Medicaid coverage during the year. Among the Discontinuous-Insured group, married individuals, those with higher education, higher incomes, and having some form of employment during the year were the characteristics associated with higher odds of Medicaid discontinuity, whereas minorities, those who were married, SSI/TANF beneficiaries, Medicaid managed care enrollees, and those with respiratory illnesses were associated with lower odds of Medicaid discontinuity. These findings may have highlighted racial and ethnic differences in Medicaid continuity; for instance, low-income minorities have been known to have lower incomes and education, and may be more likely to be unemployed, resulting in greater continuity in their Medicaid coverage compared to their Caucasian counterparts. An interesting finding was the lower odds of Medicaid discontinuity among Medicaid managed care enrollees, which may be the result of better outreach efforts by managed care organizations, although they may be incentivized to do so given they are paid on a per-member basis by Medicaid. ${ }^{34}$

When examining the associations between Medicaid discontinuity, medication adherence, and medication utilization, as part of the second aim of this dissertation, it was found that although the point estimate for overall average adherence to frequently prescribed medications was lower for the Discontinuous-Uninsured, the estimate was not statistically significantly different from the Continuous Medicaid group. This study found lower adherence to sulfonylureas among the Discontinuous-Uninsured compared to the Continuous Medicaid, which may result in higher health care costs in this subpopulation. ${ }^{113}$ The Discontinuous-Insured were found to have higher odds of adherence to ACEI/ARB and calcium channel blockers, which could have been contributed by better access to these medications through other insurance programs. The odds of clinically adequate adherence to diuretics, however, was found to be significantly 
lower among the Discontinuous-Insured, which is traditionally used as a first-line antihypertensive therapy. Medication utilization on the other hand, both all-cause and disease-specific, was found to be significantly lower among both the discontinuous Medicaid groups compared to the Continuous Medicaid. On average, the DiscontinuousUninsured had 12 fewer drug fills per person for all medications, and 2.3 fewer fills per person for CVD-specific medications. Even the Discontinuous-Insured had 6.5 fewer fills, and 2.8 fewer fills per person, for all-cause and disease-specific medications, respectively. Given the significantly lower prescription drug fills among the discontinuous Medicaid groups, this study may have overestimated adherence to medications, which may also be lower among populations lacking continuous Medicaid coverage.

Finally, as part of the third aim, this study examined associations between Medicaid discontinuity, access to care, preventive services use, and health care utilization. Access to care was poor among the discontinuous Medicaid populations, with these groups having lower odds of having usual source of care, and higher odds for experiencing difficulties in obtaining needed medical care and medications. The odds of routine medical checkups were also found to be lower among the DiscontinuousUninsured, indicating inadequate medical checkups which are critical for early detection and management of chronic conditions. Incidences of disease-specific hospitalizations, both inpatient and ER visits, were found to be significantly higher among the discontinuous Medicaid populations compared to those with continuous Medicaid. Although ER use has been found to be higher among individuals who gain Medicaid coverage, as ER may be a usual source of care for certain vulnerable populations even for conditions that can be managed in outpatient setting, the increased hospitalizations are more likely due to poor management of CVD or high-risk conditions for CVD resulting from poor access to care and inadequate pharmacotherapy. The reason being, when individuals are asked about whether or not they have a usual source of care, the options include ER as well, apart from provider offices, outpatient and other clinic settings. Moreover, all-cause office-based provider visits were lower, whereas all-cause hospital outpatient visits were higher among the Discontinuous-Uninsured, which may indicate inability to seek care and build patient-provider relationships with a usual source of primary care physician, and overreliance on the hospital clinical care setting. It may also be a result of lower acceptance of Medicaid patients by private practice physicians, however, the comparison group is those with continuous Medicaid coverage, which would have had worse primary care office visits if lack of acceptance were predominant and prevalent. These findings indicate that Medicaid discontinuity among low-income CVD or high risk chronic condition patients is associated with poor access to care, inadequate medical checkups, lower prescription drug utilization and poor adherence to certain commonly prescribed therapeutic drug classes, higher CVD-related hospitalizations and ER visits, lower overall physician office visits to seek primary care, and a greater reliance on hospital outpatient setting. 


\section{IMPLICATIONS FOR POLICY}

Gaps in Medicaid coverage may have deleterious health consequences for lowincome enrollees, and this study found that the health care access, and utilization outcomes, and medication consumption behaviors among adults with CVD or high-risk conditions for CVD may be adversely impacted due to instability in Medicaid coverage. Continuity of Medicaid, especially for those who continue to be eligible for coverage, represents a cost-effective policy solution, and lead to greater effectiveness of the prevention and treatment services and resources, especially among low-income diseased populations. State Medicaid agencies have a policy option of guaranteeing enrollment for adults in Medicaid until the next reenrollment and eligibility determination cycle, and have the duration of these cycles to be six months or annual. This would significantly reduce within enrollment period churning and ensure better continuity of Medicaid coverage, and in turn the continuity of care afforded by it. On a long term basis, cost reductions may be realized due to providing better continuity in Medicaid coverage to non-elderly adults, especially if the administrative costs associated with frequent reenrollment are higher. The immediate impact of prolonged continuity of Medicaid may however, result in a higher financial burden on the states, which may be a reason for the stringent administrative regulations governing Medicaid coverage for none-elderly adults. If that may be the case, then rather than a blanket policy that provides guaranteed Medicaid enrollment to all non-elderly adults, state Medicaid agencies may focus on specific subpopulations suffering from chronic, debilitating disease conditions, who often require prolonged medical care to manage their conditions and comorbidities, and for whom having continuous Medicaid coverage may prove to be more effective, and costeffective. Indeed, the present study showed that adults with CVD or high-risk conditions for CVD had greater access to care problems, substandard medication adherence, inadequate preventive care and primary care, and higher incidences of hospitalizations associated Medicaid discontinuity, after adjusting for a host of demographic, socioeconomic, health-related and Medicaid eligibility-related covariates in order to obtain robust estimates. And although this study did not examine individuals with other disease conditions such as respiratory illnesses, HIV/AIDS, mental illnesses, these subpopulations too may experience similar negative outcomes of poor access to and use of health care resources. Therefore, policies to stabilize Medicaid coverage among the low-income population with chronic, debilitating disease conditions, may provide more bang for the buck.

Instability in insurance, both public and private, have negative impact on health and well-being of individuals as has been established by studies above. Although there has not been any positive impact of this loss of and gaps in coverage investigated on patients and society, there are some aspects or reasons of that contribute to churning in insurance or Medicaid that are needed for the benefit of the society. One is the need for deductibles and co-payments for insurance programs. Studies have shown that inability to afford paying for deductibles and co-payments is one of the reasons that people opt out of insurance plans, or do not enroll for public health plans. Although high deductibles and co-pays have a negative effect on individuals' utilization of needed care, complete elimination of these payment structures might also be detrimental. This is because with 
"no skin in the game", there will the likelihood of what economists call "moral hazard", that is individuals will consume more health care than what they need resulting in inefficient allocation and spending of resources and excessive health care burden on the society. In order to promote rational utilization of health care resources such contribution from health plan enrollees is needed; the challenge to determining the "sweet spot" or the right balance that would ensure that it does not exert a tremendous financial burden. Another aspect is the requirement for sufficient documentation to ensure that meanstested insurance plans enroll individuals who truly qualify for the health plans, and prevent any fraud which may divert needed health care resources to individuals who may not need them. Once again, rather than getting rid of documentation requirements, the process should be made less burdensome, with greater choices or avenues given to patients, such as online enrollment, and longer time periods, and note having short periodic reporting requirements to report eligibility. Also, provisions that enable smooth transitions from public to private health insurance, or vice versa, especially for lowincome population, can also minimize churning and gaps, while still ensuring that minimum checks and balances of determining eligibility are in place to prevent wastage of much needed health care resources.

As part of the 2010 PPACA provisions of Medicaid expansion, states have the option for expanding Medicaid coverage to their non-elderly adult residents with incomes at or below 138\% FPL. As of May 2015, however, 18 states have chosen status quo over adopting these provisions and expanding Medicaid coverage, which would result in continuation of these issues of instability of Medicaid in none-elderly adults and the resulting adverse impact on continuity and quality of care and disease management. ${ }^{49}$ Even under PPACA provisions, states will be required to have 12 month recertification periods, meaning individuals will have to reenroll in Medicaid annually; however, even within this enrolled period, individuals and families may lose coverage midway if their incomes and other criteria change. For these states, ensuring continuous Medicaid coverage, at least for the diseased vulnerable populations could be a policy initiative that can be adopted to ensure better health outcomes among these populations, and possibly lower financial burden on the health care system due to providing high cost care resulting from avoidable hospitalizations. Due to competing priorities, and even among the health care sector, competing health priorities, resources available for management of these conditions may be limited and policy initiatives to ensure continuity of insurance coverage among low-income adult populations may be lacking. Failure to address issues of Medicaid coverage continuity among adults with complex chronic conditions such as CVD, may lead to large increases in prevalence of avoidable chronic conditions, which may exert a tremendous burden on the health care system and the economy.

\section{IMPLICATIONS FOR FUTURE RESEARCH}

The present study found strong associations between Medicaid discontinuity and the negative access to care and health care resource utilization outcomes among the lowincome CVD adult population. However, due to data limitations, we were unable to establish temporality between Medicaid discontinuity and various outcomes under 
investigation. Moreover, the reasons for losing or gaps in Medicaid coverage were not known. Future research may incorporate these shortcomings to obtain a holistic view of the outcomes associated, and possibly caused by disruptions in Medicaid coverage. Using state-specific eligibility would also be able to better control for state-level differences in observed and unobserved characteristics that may not have been adequately controlled by geographic region.

Costs associated with Medicaid discontinuity are an important outcome, and may be of more interest to policymakers. The obvious follow-up to this study would examine the costs, including total costs and costs specific to each health care services, to provide a comprehensive picture of the impact of Medicaid discontinuity on health care resources and costs, from the health care system perspective or payer perspective. Disruptions in care continuity associated with poor access to care, lack of preventive care and primary care, and the resulting increased hospitalizations and ER visits may lead to significant increases in health care costs. And with a significant increase ER use by the uninsured to obtain their usual care, or due hospitalizations resulting from chronic disease exacerbations, the financial burden may increase tremendously on the health care providers and hospitals due to provision of uncompensated care. The insured may end up subsidizing a significant portion of the uncompensated care costs through their insurance plans, and related out-of-pocket expenditures due to cost shifting. This vicious cycle may not only exert a financial strain on providers and payers, but on patients as well.

Examinations of cost implications due to Medicaid discontinuity from not just payer or provider perspective, but patient perspective may also provide meaningful information of the impact of Medicaid discontinuity, and produce a complete and comprehensive picture of the implications of Medicaid discontinuity among the non-elderly adult population, or subpopulations of these with chronic conditions such as CVD or several of its high-risk conditions. 


\section{LIST OF REFERENCES}

1. Enthoven AC. Integrated delivery systems: the cure for fragmentation. The American journal of managed care. Dec 2009;15(10 Suppl):S284-290.

2. Health Insurance Coverage of the Total Population. http://kff.org/other/stateindicator/total-population/. Accessed August 19, 2014.

3. Fronstin P. The Impact of the Recession on Employment-Based Health Coverage EBRI Issue Brief, no. 342 2010; http://www.ebri.org/pdf/briefspdf/EBRI IB 052010 No342_Recssn-HlthBens.pdf. Accessed August 19, 2014.

4. Gould E. Issue Brief: The Chronic Problem of Declining Health Coverage. 2004; http://www.epi.org/publication/issuebrief202/. Accessed August, 19, 2014.

5. Medicaid: A Primer. 2013; https://kaiserfamilyfoundation.files.wordpress.com/2010/06/7334-05.pdf. Accessed May 1, 2014.

6. State Health Facts. Medicaid and CHIP Indicators. http://kff.org/statecategory/medicaid-chip/. Accessed August, 19, 2014.

7. Kaplan L. A primer on Medicaid and its expansion in 2014. The Nurse practitioner. Dec 10 2012;37(12):7-8.

8. $\mathrm{Ku} \mathrm{L}$, ; Steinmetz, E. Bridging the Gap: Continuity and Quality of Coverage in Medicaid 2013; http://ccf.georgetown.edu/wp-content/uploads/2013/09/GWContinuity-Report-9-10-13.pdf. Accessed August, 19, 2014.

9. Ku LM, P.; Pervez, F.; Rosenbaum, S. Improving Medicaid's Continuity of Coverage and Quality Of Care. 2009; http://www.communityplans.net/Portals/0/ACAP\%20Docs/Improving\%20Medica id\%20Final\%20070209.pdf. Accessed August, 19, 2014.

10. Harman JS, Manning WG, Lurie N, Christianson JB. Association between interruptions in medicaid coverage and use of inpatient psychiatric services. Psychiatric services. Jul 2003;54(7):999-1005.

11. Koroukian SM. Screening mammography was used more, and more frequently, by longer than shorter term Medicaid enrollees. Journal of clinical epidemiology. Aug 2004;57(8):824-831.

12. Koroukian SM. Assessing the effectiveness of Medicaid in breast and cervical cancer prevention. Journal of public health management and practice : JPHMP. Jul-Aug 2003;9(4):306-314.

13. Harman JS, Hall AG, Zhang J. Changes in health care use and costs after a break in Medicaid coverage among persons with depression. Psychiatric services. Jan 2007;58(1):49-54.

14. Bindman AB, Chattopadhyay A, Auerback GM. Interruptions in Medicaid coverage and risk for hospitalization for ambulatory care-sensitive conditions. Annals of internal medicine. Dec 16 2008;149(12):854-860.

15. Banerjee R, Ziegenfuss JY, Shah ND. Impact of discontinuity in health insurance on resource utilization. BMC health services research. 2010;10:195. 
16. Murphy SL, Xu J, Kochanek KD. Deaths: final data for 2010. National vital statistics reports : from the Centers for Disease Control and Prevention, National Center for Health Statistics, National Vital Statistics System. May 8 2013;61(4):1117.

17. Global Burden of Metabolic Risk Factors for Chronic Diseases C. Cardiovascular disease, chronic kidney disease, and diabetes mortality burden of cardiometabolic risk factors from 1980 to 2010: a comparative risk assessment. The lancet. Diabetes \& endocrinology. Aug 2014;2(8):634-647.

18. Yusuf S, Reddy S, Ounpuu S, Anand S. Global burden of cardiovascular diseases: part I: general considerations, the epidemiologic transition, risk factors, and impact of urbanization. Circulation. Nov 27 2001;104(22):2746-2753.

19. Mozaffarian D, Benjamin EJ, Go AS, et al. Heart disease and stroke statistics-2015 update: a report from the American Heart Association. Circulation. Jan 27 2015;131(4):e29-322.

20. The Role of Medicaid for People with Cardiovascular Diseases. 2012; https://kaiserfamilyfoundation.files.wordpress.com/2013/01/8383_cd.pdf Accessed August 1, 2014.

21. Mensah GA, Brown DW. An overview of cardiovascular disease burden in the United States. Health affairs. Jan-Feb 2007;26(1):38-48.

22. Mendes E. Preventable Chronic Conditions Plague Medicaid Population. 2013; http://www.gallup.com/poll/161615/preventable-chronic-conditions-plaguemedicaid-population.aspx. Accessed October, 20, 2014.

23. What is Cardiovascular Disease? 2015; http://www.heart.org/HEARTORG/Caregiver/Resources/WhatisCardiovascularDi sease/What-is-Cardiovascular-Disease_UCM_301852_Article.jsp. Accessed October 20,, 2014.

24. Cardiovascular diseases (CVDs). 2015; http://www.who.int/mediacentre/factsheets/fs317/en/. Accessed October, 20, 2014.

25. Grundy SM, Pasternak R, Greenland P, Smith S, Jr., Fuster V. Assessment of cardiovascular risk by use of multiple-risk-factor assessment equations: a statement for healthcare professionals from the American Heart Association and the American College of Cardiology. Circulation. Sep 28 1999;100(13):14811492.

26. Sarnak MJ, Levey AS, Schoolwerth AC, et al. Kidney disease as a risk factor for development of cardiovascular disease: a statement from the American Heart Association Councils on Kidney in Cardiovascular Disease, High Blood Pressure Research, Clinical Cardiology, and Epidemiology and Prevention. Circulation. Oct 28 2003;108(17):2154-2169.

27. Knapton M, Lewin RJ, Doherty PJ. Long-term cardiovascular conditions: the role of primary care. The British journal of general practice : the journal of the Royal College of General Practitioners. Nov 2011;61(592):659-660.

28. Shin DW, Cho J, Yang HK, et al. Impact of continuity of care on mortality and health care costs: a nationwide cohort study in Korea. Annals of family medicine. Nov-Dec 2014;12(6):534-541. 
29. Facts: Critical Coverage for Heart Health: Medicaid and Cardiovascular Disease 2011; http://www.heart.org/idc/groups/heartpublic/@wcm/@adv/documents/downloadable/ucm_428187.pdf. Accessed October, 20, 2014.

30. The Role of Medicaid for Adults with Chronic Illnesses 2012; https://kaiserfamilyfoundation.files.wordpress.com/2013/01/8383.pdf. Accessed August, 19, 2014.

31. Ayanian JZ, Weissman JS, Schneider EC, Ginsburg JA, Zaslavsky AM. Unmet health needs of uninsured adults in the United States. Jama. Oct 25 2000;284(16):2061-2069.

32. Fields BE, Bell JF, Moyce S, Bigbee JL. The impact of insurance instability on health service utilization: does non-metropolitan residence make a difference? The Journal of rural health : official journal of the American Rural Health Association and the National Rural Health Care Association. Jan 2015;31(1):2734.

33. Morreale MC, English A. Eligibility and enrollment of adolescents in Medicaid and SCHIP: recent progress, current challenges. The Journal of adolescent health : official publication of the Society for Adolescent Medicine. Jun 2003;32(6 Suppl):25-39.

34. Sommers BD. From Medicaid to uninsured: drop-out among children in public insurance programs. Health services research. Feb 2005;40(1):59-78.

35. Summer LM, C. Instability of public health insurance coverage for children and their families: Causes, consequences, and remedies 2006; http://www.commonwealthfund.org/ /media/files/publications/fundreport/2006/jun/instability-of-public-health-insurance-coverage-for-children-andtheir-families--causes--consequence/summer instabilitypubhltinschildren 935pdf.pdf. Accessed October 20, 2014.

36. Ellwood MRL, K. On and Off Medicaid: Enrollment Patterns for California and Florida in 1995. 1999; http://webarchive.urban.org/UploadedPDF/occa27.pdf. Accessed October, 20, 2014.

37. Ku LR, D. C. Staying covered: The importance of retaining health insurance for low-income families. 2002; http://www.commonwealthfund.org/ /media/files/publications/fundreport/2002/dec/staying-covered--the-importance-of-retaining-health-insurancefor-low-income-families/ku stayingcovered 586-pdf.pdf. Accessed October, 20, 2014.

38. Sommers BD. Loss of health insurance among non-elderly adults in Medicaid. Journal of general internal medicine. Jan 2009;24(1):1-7.

39. Survey Background: Medicaid Expenditure Panel Survey. 2009; http://meps.ahrq.gov/mepsweb/about_meps/survey back.jsp. Accessed May, 15, 2013.

40. MEPS HC-146 2011 Medical Conditions. 2013; http://meps.ahrq.gov/mepsweb/data_stats/download_data/pufs/h146/h146doc.pdf. Accessed May, 15, 2013. 
41. MEPS-HC Sample Design and Collection Process. 2015;

http://www.meps.ahrq.gov/survey comp/hc data collection.jsp. Accessed April 6, 2015.

42. MEPS HC-147 2011 Full Year Consolidated Data File. 2013; http://meps.ahrq.gov/mepsweb/data_stats/download_data/pufs/h147/h147doc.pdf.

43. Shirneshan E, Bailey J, Relyea G, Franklin BE, Solomon DK, Brown LM. Incremental direct medical expenditures associated with anxiety disorders for the U.S. adult population: evidence from the Medical Expenditure Panel Survey. Journal of anxiety disorders. Oct 2013;27(7):720-727.

44. Report to the Congress on Medicaid and CHIP. 2014; https://www.macpac.gov/wp-content/uploads/2015/01/2014-03-

14 Macpac Report.pdf. Accessed May, 15, 2013.

45. Short PF, Graefe DR. Battery-powered health insurance? Stability in coverage of the uninsured. Health affairs. Nov-Dec 2003;22(6):244-255.

46. Ellwood M. The Medicaid eligibility maze: Coverage expands, but enrollment problems persist. Findings from a five state study. 1999; https://kaiserfamilyfoundation.files.wordpress.com/1999/08/2164-the-medicaideligibility-maze-coverage-expands-but-problems-persist.pdf. Accessed May, 15, 2013.

47. Riley TP, C.; Perry, M.; Kannel, S. Why Eligible Children Lose or Leave SCH1P. Findings from a comprehensive study of retention and disenrollment 2002; http://www.nashp.org/sites/default/files/SCHIP lose or leave.pdf. Accessed May, 15, 2013.

48. Kenney GM, Lynch V, Haley J, Huntress M. Variation in Medicaid eligibility and participation among adults: implications for the Affordable Care Act. Inquiry : a journal of medical care organization, provision and financing. Fall 2012;49(3):231-253.

49. Status of State Action on the Medicaid Expansion Decision. 2015; http://kff.org/health-reform/state-indicator/state-activity-around-expandingmedicaid-under-the-affordable-care-act/. Accessed May, 26, 2015.

50. Questions and Answers on the Premium Tax Credit. 2015; http://www.irs.gov/Affordable-Care-Act/Individuals-and-Families/Questions-andAnswers-on-the-Premium-Tax-Credit. Accessed May, 30, 2015.

51. Sommers BD, Graves JA, Swartz K, Rosenbaum S. Medicaid and marketplace eligibility changes will occur often in all states; policy options can ease impact. Health affairs. Apr 2014;33(4):700-707.

52. Sommers BD, Rosenbaum S. Issues in health reform: how changes in eligibility may move millions back and forth between medicaid and insurance exchanges. Health affairs. Feb 2011;30(2):228-236.

53. Informing CHIP and Medicaid Outreach and Education 2011; http://www.insurekidsnow.gov/professionals/CHIP-Medicaid-SurveyTopline.pdf. Accessed May, 1, 2015.

54. Comparing Federal Government Surveys that Count the Uninsured. 2010; http://www.rwjf.org/content/dam/farm/reports/issue briefs/2010/rwjf66804. Accessed May, 1, 2015. 
55. Lozano R, Naghavi M, Foreman K, et al. Global and regional mortality from 235 causes of death for 20 age groups in 1990 and 2010: a systematic analysis for the Global Burden of Disease Study 2010. Lancet. Dec 15 2012;380(9859):20952128.

56. Prevention of Cardiovascular Disease: Pocket Guidelines for Assessment and Management of Cardiovascular Risk 2007; http://www.who.int/cardiovascular diseases/guidelines/PocketGL.ENGLISH.AF R-D-E.rev1.pdf. Accessed March, 1, 2015.

57. Califf RM, Armstrong PW, Carver JR, D'Agostino RB, Strauss WE. 27th Bethesda Conference: matching the intensity of risk factor management with the hazard for coronary disease events. Task Force 5. Stratification of patients into high, medium and low risk subgroups for purposes of risk factor management. Journal of the American College of Cardiology. Apr 1996;27(5):1007-1019.

58. Parikh NI, Hwang SJ, Larson MG, Meigs JB, Levy D, Fox CS. Cardiovascular disease risk factors in chronic kidney disease: overall burden and rates of treatment and control. Archives of internal medicine. Sep 25 2006;166(17):18841891.

59. Wolf-Maier K, Cooper RS, Kramer H, et al. Hypertension treatment and control in five European countries, Canada, and the United States. Hypertension. Jan 2004;43(1):10-17.

60. Wong ND, Lopez V, Tang S, Williams GR. Prevalence, treatment, and control of combined hypertension and hypercholesterolemia in the United States. The American journal of cardiology. Jul 15 2006;98(2):204-208.

61. Boswell KACCLB, S. P.; Eaddy, M. T.; Ron Cantrell, C. R. Associating Medication Adherence With Improved Outcomes: A Systematic Literature Review. Am J Pharm Benefits. Jul 2012;4(4):e97-e108.

62. Ho PM, Bryson CL, Rumsfeld JS. Medication adherence: its importance in cardiovascular outcomes. Circulation. Jun 16 2009;119(23):3028-3035.

63. McDermott MM, Schmitt B, Wallner E. Impact of medication nonadherence on coronary heart disease outcomes. A critical review. Archives of internal medicine. Sep 22 1997;157(17):1921-1929.

64. Sokol MC, McGuigan KA, Verbrugge RR, Epstein RS. Impact of medication adherence on hospitalization risk and healthcare cost. Medical care. Jun 2005;43(6):521-530.

65. Bailey JE, Wan JY, Tang J, Ghani MA, Cushman WC. Antihypertensive medication adherence, ambulatory visits, and risk of stroke and death. Journal of general internal medicine. Jun 2010;25(6):495-503.

66. Ho PM, Rumsfeld JS, Masoudi FA, et al. Effect of medication nonadherence on hospitalization and mortality among patients with diabetes mellitus. Archives of internal medicine. Sep 25 2006;166(17):1836-1841.

67. Smith SR, Kirking DM, Acsus. The effect of insurance coverage changes on drug utilization in HIV disease. Journal of acquired immune deficiency syndromes. Oct $12001 ; 28(2): 140-149$.

68. Schneider AE, R.; Garfield, R. Chapter 1: Medicaid Eligibility. https://kaiserfamilyfoundation.files.wordpress.com/2013/05/mrbeligibility.pdf. Accessed May, 1, 2015. 
69. Choudhry NK, Shrank WH, Levin RL, et al. Measuring concurrent adherence to multiple related medications. The American journal of managed care. Jul 2009;15(7):457-464.

70. Karve S, Cleves MA, Helm M, Hudson TJ, West DS, Martin BC. Prospective validation of eight different adherence measures for use with administrative claims data among patients with schizophrenia. Value in health: the journal of the International Society for Pharmacoeconomics and Outcomes Research. Sep 2009;12(6):989-995.

71. Zwilling ML. "Negative Binomial Regression. The Mathematica Journal Volume 15 2013; http://www.mathematica-journal.com/2013/06/negative-binomialregression/. Accessed May, 1, 2015.

72. Williams R. Marginal Effects for Continuous Variables. 2015; https://www3.nd.edu/ rwilliam/stats3/Margins02.pdf. Accessed May, 1, 2015.

73. Adherence to Long-Term Therapies: Evidence for action. 2003; http://www.who.int/chp/knowledge/publications/adherence_full_report.pdf. Accessed May, 1, 2015.

74. Mandrola J. Growing Doubt on Statin Drugs: The Problem of Drug-Lifestyle Interaction. 2014. http://www.medscape.com/viewarticle/827675\#vp_1. Accessed May 1, 2015.

75. Baicker K, Taubman SL, Allen HL, et al. The Oregon experiment--effects of Medicaid on clinical outcomes. The New England journal of medicine. May 2 2013;368(18):1713-1722.

76. Medicaid Benefits: Prescription Drugs. 2012; http://kff.org/medicaid/stateindicator/prescription-drugs/\#notes. Accessed May 1, 2015.

77. Rice T, Lavarreda SA, Ponce NA, Brown ER. The impact of private and public health insurance on medication use for adults with chronic diseases. Medical care research and review : MCRR. Apr 2005;62(2):231-249.

78. Glader EL, Sjolander M, Eriksson M, Lundberg M. Persistent use of secondary preventive drugs declines rapidly during the first 2 years after stroke. Stroke; $a$ journal of cerebral circulation. Feb 2010;41(2):397-401.

79. Jackevicius CA, Li P, Tu JV. Prevalence, predictors, and outcomes of primary nonadherence after acute myocardial infarction. Circulation. Feb 26 2008;117(8):1028-1036.

80. Newby LK, LaPointe NM, Chen AY, et al. Long-term adherence to evidencebased secondary prevention therapies in coronary artery disease. Circulation. Jan 17 2006;113(2):203-212.

81. Bramley TJ, Gerbino PP, Nightengale BS, Frech-Tamas F. Relationship of blood pressure control to adherence with antihypertensive monotherapy in 13 managed care organizations. Journal of managed care pharmacy : JMCP. Apr 2006;12(3):239-245.

82. Vrijens B, Vincze G, Kristanto P, Urquhart J, Burnier M. Adherence to prescribed antihypertensive drug treatments: longitudinal study of electronically compiled dosing histories. Bmj. May 17 2008;336(7653):1114-1117.

83. Gislason GH, Rasmussen JN, Abildstrom SZ, et al. Persistent use of evidencebased pharmacotherapy in heart failure is associated with improved outcomes. Circulation. Aug 14 2007;116(7):737-744. 
84. Granger BB, Swedberg K, Ekman I, et al. Adherence to candesartan and placebo and outcomes in chronic heart failure in the CHARM programme: double-blind, randomised, controlled clinical trial. Lancet. Dec 10 2005;366(9502):2005-2011.

85. Dailey G, Kim MS, Lian JF. Patient compliance and persistence with antihyperglycemic drug regimens: evaluation of a medicaid patient population with type 2 diabetes mellitus. Clinical therapeutics. Aug 2001;23(8):1311-1320.

86. Khanna R, Pace PF, Mahabaleshwarkar R, Basak RS, Datar M, Banahan BF. Medication adherence among recipients with chronic diseases enrolled in a state Medicaid program. Population health management. Oct 2012;15(5):253-260.

87. Hill SC, Zuvekas SH, Zodet MW. Implications of the accuracy of MEPS prescription drug data for health services research. Inquiry : a journal of medical care organization, provision and financing. Fall 2011;48(3):242-259.

88. Total Monthly Medicaid and CHIP Enrollment. 2015; http://kff.org/healthreform/state-indicator/total-monthly-medicaid-and-chip-enrollment/. Accessed May 1, 2015.

89. The importance of primary care physicians as the usual source of healthcare in the achievement of prevention goals. American family physician. Nov 1 2000;62(9):1968.

90. Corbie-Smith G, Flagg EW, Doyle JP, O'Brien MA. Influence of usual source of care on differences by race/ethnicity in receipt of preventive services. Journal of general internal medicine. Jun 2002;17(6):458-464.

91. Ettner SL. The relationship between continuity of care and the health behaviors of patients: does having a usual physician make a difference? Medical care. Jun 1999;37(6):547-555.

92. Weissman JS, Stern R, Fielding SL, Epstein AM. Delayed access to health care: risk factors, reasons, and consequences. Annals of internal medicine. Feb 15 1991;114(4):325-331.

93. Kasper JD, Giovannini TA, Hoffman C. Gaining and losing health insurance: strengthening the evidence for effects on access to care and health outcomes. Medical care research and review : MCRR. Sep 2000;57(3):298-318; discussion 319-225.

94. Schoen C, DesRoches C. Uninsured and unstably insured: the importance of continuous insurance coverage. Health services research. Apr 2000;35(1 Pt 2):187-206.

95. Sudano JJ, Jr., Baker DW. Intermittent lack of health insurance coverage and use of preventive services. American journal of public health. Jan 2003;93(1):130137.

96. Bindman AB, Grumbach K, Osmond D, Vranizan K, Stewart AL. Primary care and receipt of preventive services. Journal of general internal medicine. May 1996;11(5):269-276.

97. Mauskopf J, Turner BJ, Markson LE, Houchens RL, Fanning TR, McKee L. Patterns of ambulatory care for AIDS patients, and association with emergency room use. Health services research. Oct 1994;29(4):489-510.

98. Parchman ML, Culler S. Primary care physicians and avoidable hospitalizations. The Journal of family practice. Aug 1994;39(2):123-128. 
99. Key Lessons from Medicaid and CHIP for Outreach and Enrollment Under the Affordable Care Act. Getting into Gear for 2014 2013; https://kaiserfamilyfoundation.files.wordpress.com/2013/06/8445-key-lessonsfrom-medicaid-and-chip.pdf. Accessed May 1, 2015.

100. Short PF, Graefe DR, Schoen C. Churn, churn, churn: how instability of health insurance shapes America's uninsured problem. Issue brief. Nov 2003(688):1-16.

101. Hall AG, Harman JS, Zhang J. Lapses in Medicaid coverage: impact on cost and utilization among individuals with diabetes enrolled in Medicaid. Medical care. Dec 2008;46(12):1219-1225.

102. Wang W, Albert JM. Estimation of mediation effects for zero-inflated regression models. Statistics in medicine. Nov 20 2012;31(26):3118-3132.

103. Greene WH. Accounting for Excess Zeros and Sample Selection in Poisson and Negative Binomial Regression Models. NYU Working Paper No. EC-94-10 1994; http://papers.ssrn.com/sol3/papers.cfm?abstract_id=1293115. Accessed May 1, 2015.

104. Lurie N, Ward NB, Shapiro MF, Brook RH. Termination from Medi-Cal--does it affect health? The New England journal of medicine. Aug 16 1984;311(7):480484.

105. Lurie N, Ward NB, Shapiro MF, Gallego C, Vaghaiwalla R, Brook RH. Termination of Medi-Cal benefits. A follow-up study one year later. The New England journal of medicine. May 8 1986;314(19):1266-1268.

106. Carlson MJ, DeVoe J, Wright BJ. Short-term impacts of coverage loss in a Medicaid population: early results from a prospective cohort study of the Oregon Health Plan. Annals of family medicine. Sep-Oct 2006;4(5):391-398.

107. Coughlin TA, Long SK, Shen YC. Assessing access to care under Medicaid: evidence for the nation and thirteen states. Health affairs. Jul-Aug 2005;24(4):1073-1083.

108. Long SK, Coughlin T, King J. How well does Medicaid work in improving access to care? Health services research. Feb 2005;40(1):39-58.

109. Paradise JG, R. What is Medicaid's Impact on Access to Care, Health Outcomes, and Quality of Care? Setting the Record Straight on the Evidence. 2013; https://kaiserfamilyfoundation.files.wordpress.com/2013/08/8467-what-ismedicaids-impact-on-access-to-care1.pdf. Accessed May 1, 2015.

110. Coughlin TAL, S. K.; Clemans-Cope, L.; Resnick, D. What Difference Does Medicaid Make? Assessing Cost Effectiveness, Access, and Financial Protection under Medicaid for Low-Income Adults. 2013; https://kaiserfamilyfoundation.files.wordpress.com/2013/05/8440-whatdifference-does-medicaid-make2.pdf. Accessed May 1, 2015.

111. Taubman SL, Allen HL, Wright BJ, Baicker K, Finkelstein AN. Medicaid increases emergency-department use: evidence from Oregon's Health Insurance Experiment. Science. Jan 17 2014;343(6168):263-268.

112. Ross DCC, L. Enrolling Children and Families in Health Coverage: The Promise of Doing More. 2002; https://kaiserfamilyfoundation.files.wordpress.com/2002/05/4046.pdf. Accessed May 30, 2015. 
113. Hansen RA, Farley JF, Droege M, Maciejewski ML. A retrospective cohort study of economic outcomes and adherence to monotherapy with metformin, pioglitazone, or a sulfonylurea among patients with type 2 diabetes mellitus in the United States from 2003 to 2005. Clinical therapeutics. Jul 2010;32(7):13081319. 


\section{APPENDIX A. SUPPLEMENTAL MATERIALS FOR CHAPTER 3}

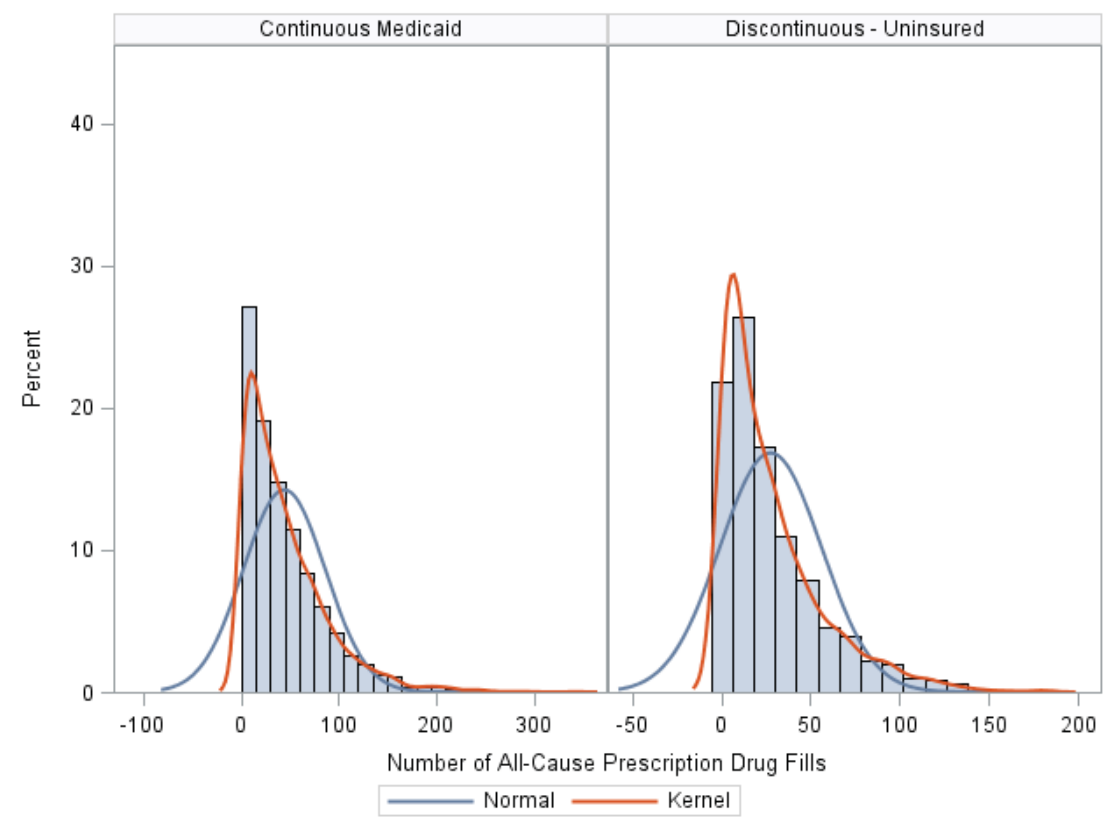

Figure A-1. Distribution of All-Cause Prescription Drug Fills among Continuous Medicaid and Discontinuous-Uninsured

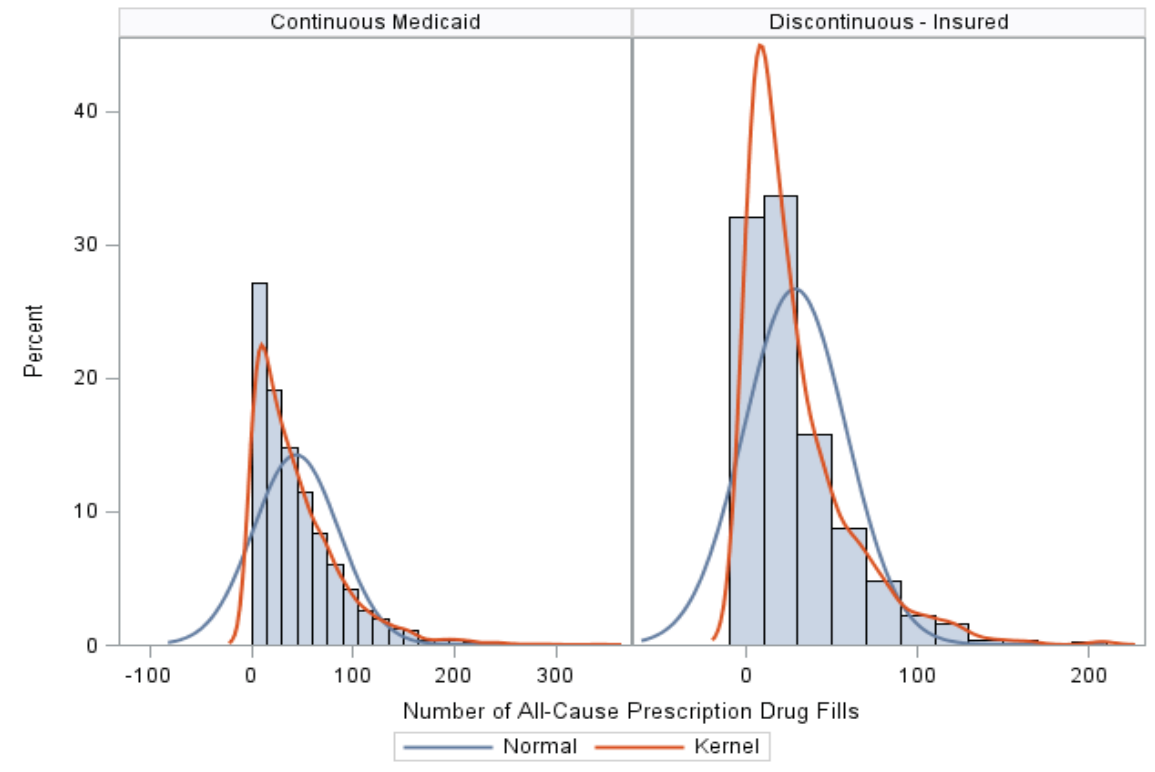

Figure A-2. Distribution of All-Cause Prescription Drug Fills among Continuous Medicaid and Discontinuous-Insured 


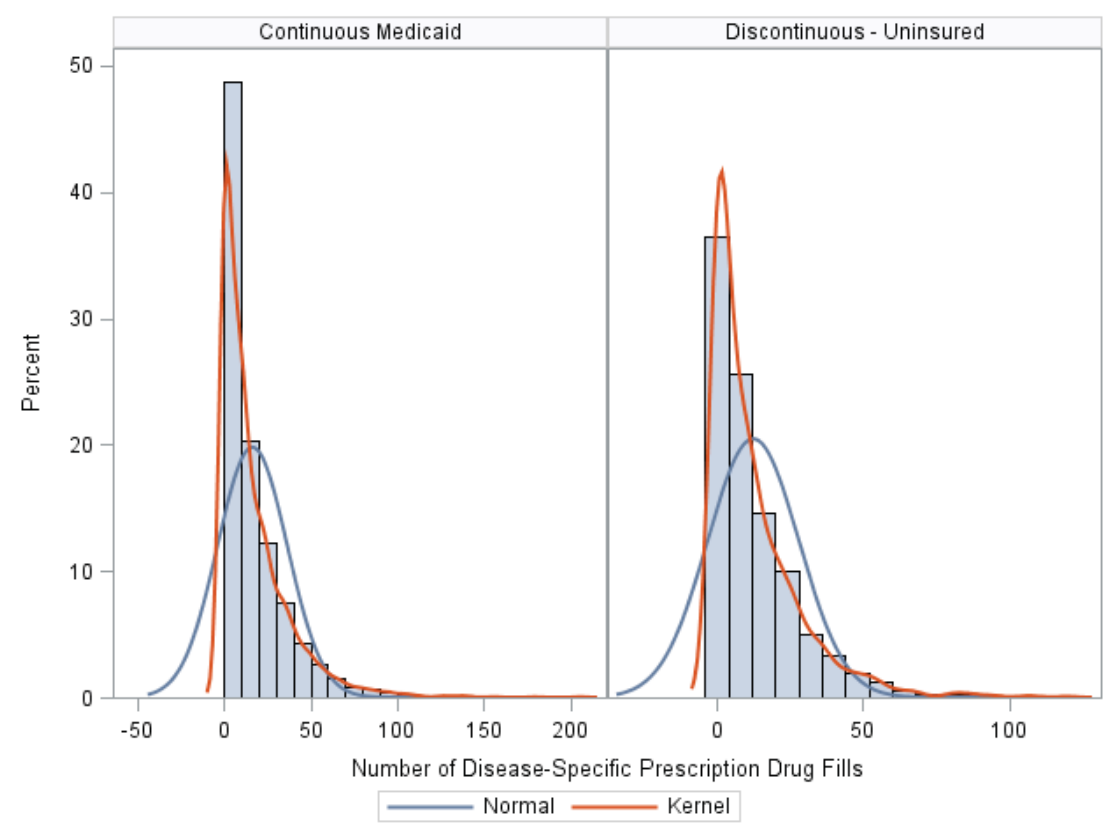

Figure A-3. Distribution of Disease-Specific Prescription Drug Fills among Continuous Medicaid and Discontinuous-Uninsured

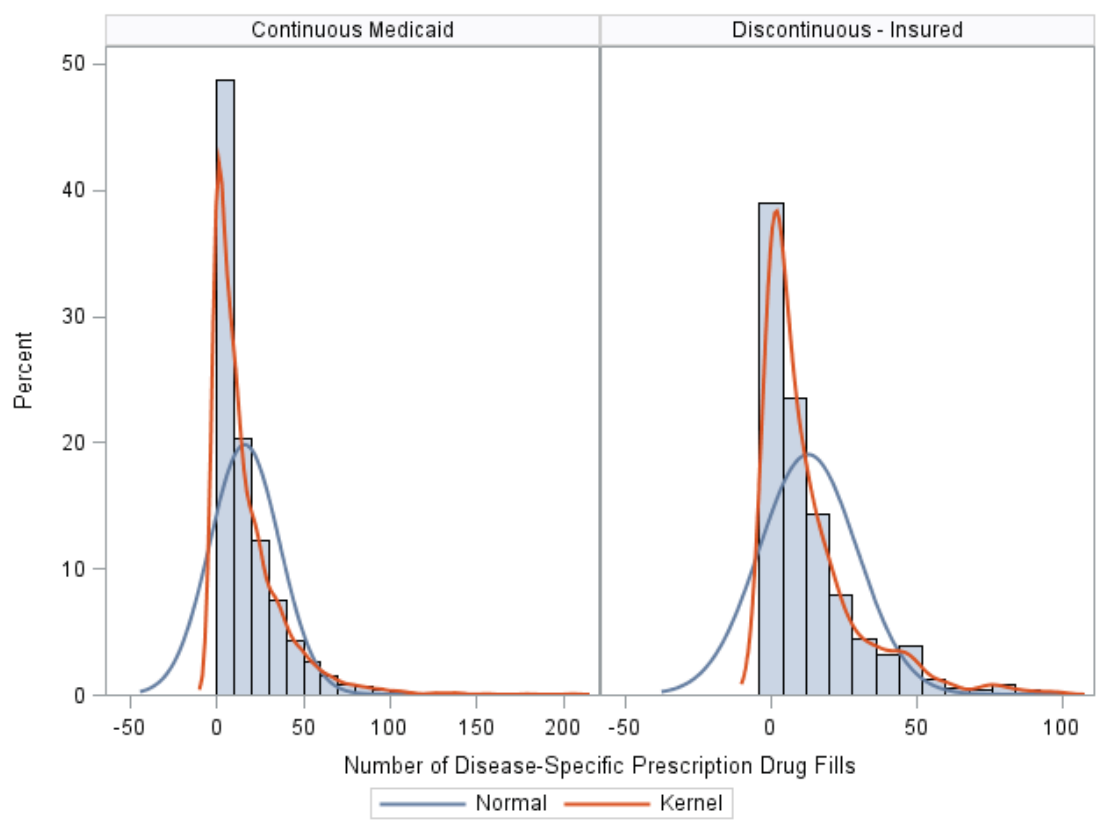

Figure A-4. Distribution of Disease-Specific Prescription Drug Fills among Continuous Medicaid and Discontinuous-Insured 
Table A-1. Characteristics Associated with Medication Adherence

\begin{tabular}{|c|c|c|}
\hline Characteristics & $\begin{array}{c}\text { Discontinuous-Uninsured } \\
\text { Odds Ratio (95\% Confidence Interval) }\end{array}$ & $\begin{array}{c}\text { Discontinuous-Insured } \\
\text { Odds Ratio (95\% Confidence Interval) }\end{array}$ \\
\hline \multicolumn{3}{|l|}{ DEMOGRAPHIC } \\
\hline \multicolumn{3}{|l|}{ Age } \\
\hline $18-34$ years & 1.00 (Reference) & 1.00 (Reference) \\
\hline $35-49$ years & $1.25(0.97-1.61)$ & $1.16(0.90-1.49)$ \\
\hline $50-64$ years & $1.26(0.97-1.63)$ & $1.22(0.93-1.59)$ \\
\hline \multicolumn{3}{|l|}{ Gender } \\
\hline Female & 1.00 (Reference) & 1.00 (Reference) \\
\hline Male & $1.11(0.91-1.35)$ & $1.09(0.88-1.35)$ \\
\hline \multicolumn{3}{|l|}{ Race/Ethnicity } \\
\hline Non-Hispanic White & 1.00 (Reference) & 1.00 (Reference) \\
\hline Non-Hispanic Black & $0.83(0.67-1.04)$ & $0.81(0.65-1.01)$ \\
\hline Hispanic & $0.86(0.68-1.09)$ & $0.79(0.61-1.02)$ \\
\hline Others & $0.74(0.53-1.02)$ & $0.74(0.54-1.01)$ \\
\hline \multicolumn{3}{|l|}{ Marital Status } \\
\hline Not Married & 1.00 (Reference) & 1.00 (Reference) \\
\hline Married & $0.96(0.79-1.17)$ & $1.05(0.86-1.30)$ \\
\hline \multicolumn{3}{|l|}{ Region } \\
\hline Northeast & 1.00 (Reference) & 1.00 (Reference) \\
\hline Midwest & $1.12(0.84-1.49)$ & $1.01(0.76-1.37)$ \\
\hline South & $1.05(0.83-1.34)$ & $1.00(0.78-1.29)$ \\
\hline
\end{tabular}


Table A-1. (Continued)

\begin{tabular}{lcc}
\hline \multicolumn{1}{c}{ Characteristics } & $\begin{array}{c}\text { Discontinuous-Uninsured } \\
\text { Odds Ratio (95\% Confidence Interval) }\end{array}$ & $\begin{array}{c}\text { Discontinuous-Insured } \\
\text { Odds Ratio (95\% Confidence Interval) }\end{array}$ \\
\hline West & $1.02(0.79-1.31)$ & $1.02(0.79-1.32)$ \\
Family Size & $0.97(0.93-1.02)$ & $0.95(0.90-1.00)$ \\
SOCIOECONOMIC & & \\
Education & 1.00 (Reference) \\
$<$ High School & $0.99(0.84-1.18)$ & 1.00 (Reference) \\
High School/GED & $0.85(0.61-1.19)$ & $0.97(0.81-1.71)$ \\
College Degree & & $0.85(0.62-1.17)$ \\
Income & 1.00 (Reference) & \\
$<100 \%$ FPL & $0.87(0.66-1.19)$ & 1.00 (Reference) \\
$100-<125 \%$ FPL & $1.11(0.93-1.33)$ & $0.84(0.61-1.15)$ \\
$\geq 125 \%$ FPL & & $1.04(0.85-1.27)$ \\
Employment & $1.00($ Reference) & \\
Always Unemployed & $1.01(0.72-1.40)$ & 1.00 (Reference) \\
Unstable Employment & $0.97(0.75-1.25)$ & $1.00(0.73-1.38)$ \\
Always Employed & & $1.01(0.79-1.30)$ \\
MSA & $1.00($ Reference) & 1.00 (Reference) \\
Non-MSA & $1.00(0.80-1.25)$ & $0.97(0.77-1.22)$ \\
MSA & &
\end{tabular}


Table A-1. (Continued)

\begin{tabular}{|c|c|c|}
\hline Characteristics & $\begin{array}{c}\text { Discontinuous-Uninsured } \\
\text { Odds Ratio ( } 95 \% \text { Confidence Interval) }\end{array}$ & $\begin{array}{c}\text { Discontinuous-Insured } \\
\text { Odds Ratio (95\% Confidence Interval) }\end{array}$ \\
\hline \multicolumn{3}{|l|}{ MEDICAID-RELATED } \\
\hline \multicolumn{3}{|l|}{ SSI/TANF } \\
\hline No & 1.00 (Reference) & 1.00 (Reference) \\
\hline Yes & $1.02(0.87-1.20)$ & $1.01(0.84-1.21)$ \\
\hline \multicolumn{3}{|l|}{ Medicaid HMO/MCO } \\
\hline No & 1.00 (Reference) & 1.00 (Reference) \\
\hline Yes & $1.15(0.97-1.36)$ & $1.10(0.92-1.31)$ \\
\hline \multicolumn{3}{|l|}{ HEALTH-RELATED } \\
\hline \multicolumn{3}{|l|}{ Perceived Health Status } \\
\hline Excellent/Very Good/Good & 1.00 (Reference) & 1.00 (Reference) \\
\hline Fair to Poor & $1.22(1.01-1.48)$ & $1.10(0.90-1.34)$ \\
\hline \multicolumn{3}{|l|}{ Perceived Mental Health } \\
\hline Excellent/Very Good/Good & 1.00 (Reference) & 1.00 (Reference) \\
\hline Fair to Poor & $1.07(0.89-1.30)$ & $1.11(0.92-1.35)$ \\
\hline \multicolumn{3}{|c|}{$\begin{array}{l}\text { CVD or High-Risk Conditions for } \\
\text { CVD }\end{array}$} \\
\hline$\leq 1$ & 1.00 (Reference) & 1.00 (Reference) \\
\hline 2 & $0.82(0.63-1.06)$ & $0.93(0.70-1.22)$ \\
\hline$\geq 3$ & $1.01(0.76-1.34)$ & $1.00(0.72-1.38)$ \\
\hline
\end{tabular}




\section{Table A-1. (Continued)}

\begin{tabular}{lcc}
\hline \multicolumn{1}{c}{ Characteristics } & $\begin{array}{c}\text { Discontinuous-Uninsured } \\
\text { Odds Ratio (95\% Confidence Interval) }\end{array}$ & $\begin{array}{c}\text { Discontinuous-Insured } \\
\text { Odds Ratio (95\% Confidence Interval) }\end{array}$ \\
\hline Mental/Substance Abuse Illnesses & 1.00 (Reference) & 1.00 (Reference) \\
No & $1.12(0.91-1.38)$ & $0.91(0.73-1.13)$ \\
Yes & & \\
Respiratory Illnesses & 1.00 (Reference) & 1.00 (Reference) \\
No & $0.88(0.70-1.10)$ & $0.81(0.63-1.04)$ \\
Yes & & \\
Arthritis/Joint Pain & $1.00($ Reference) & 1.00 (Reference) \\
No & $1.13(0.93-1.37)$ & $1.30(1.04-1.62)^{*}$ \\
Yes & $1.00(0.94-1.07)$ & $1.03(0.97-1.11)$ \\
CCI Score & & \\
\hline
\end{tabular}

Study population includes MEPS respondents from the years 2002-2011, aged 18-64 years, who either reported being diagnosed with, or reported having an event (prescription medication, or inpatient, emergency, outpatient, or office-based provider visits) associated with, 1 or more cardiovascular disease or associated risk factor comorbidities considered in this study.

$* * * P$-value $<0.001, * * P<0.01, * P<0.05$ for the difference between Continuous Medicaid vs Discontinuous-Uninsured and Continuous Medicaid vs Discontinuous-Insured groups

SE: Standard error; GED: General educational development; FPL: Federal poverty level; MSA: Metropolitan Statistical Area; SSI: Supplementary Security Income due to disability; TANF: Temporary Assistance to Needy Families program participation; HMO: Health maintenance organization; MCO: Managed care organization; CCI: Charlson Comorbidity Index score (D'Hoore adapted CCI score was calculated in this study using the 3-digit ICD-9-CM codes in MEPS). 
APPENDIX B. SUPPLEMENTAL MATERIALS FOR CHAPTER 4

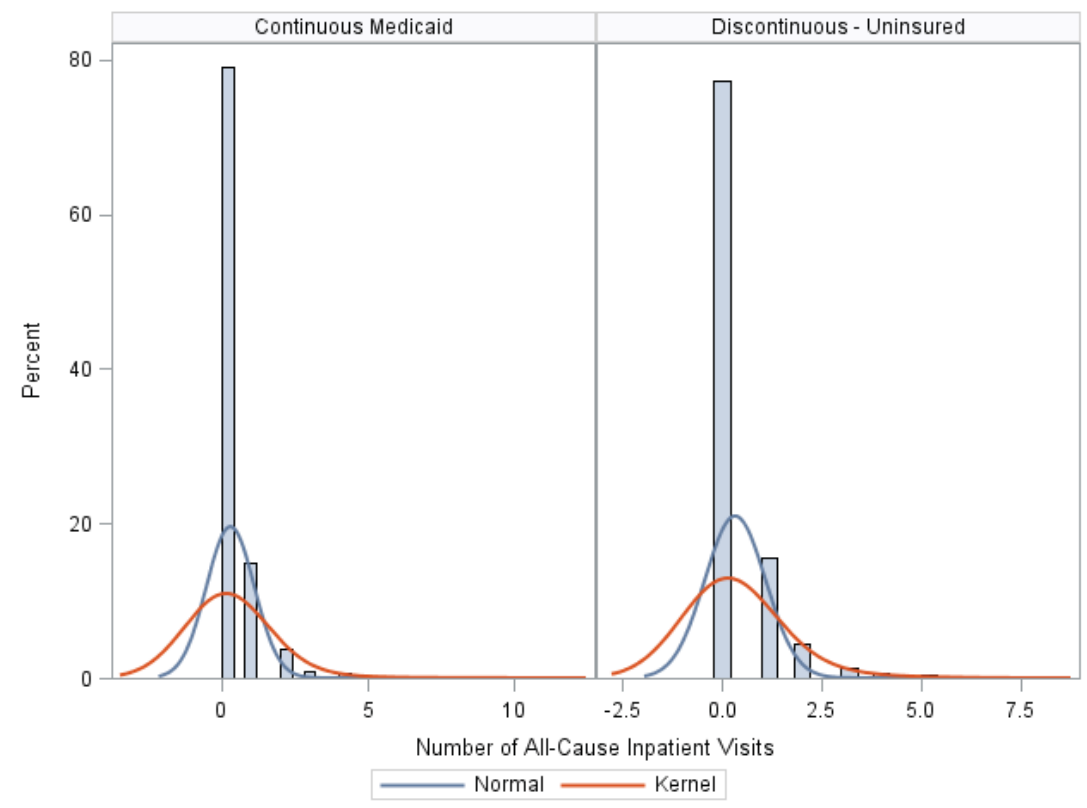

Figure B-1. Distribution of All-Cause Inpatient Visits among Continuous Medicaid and Discontinuous-Uninsured

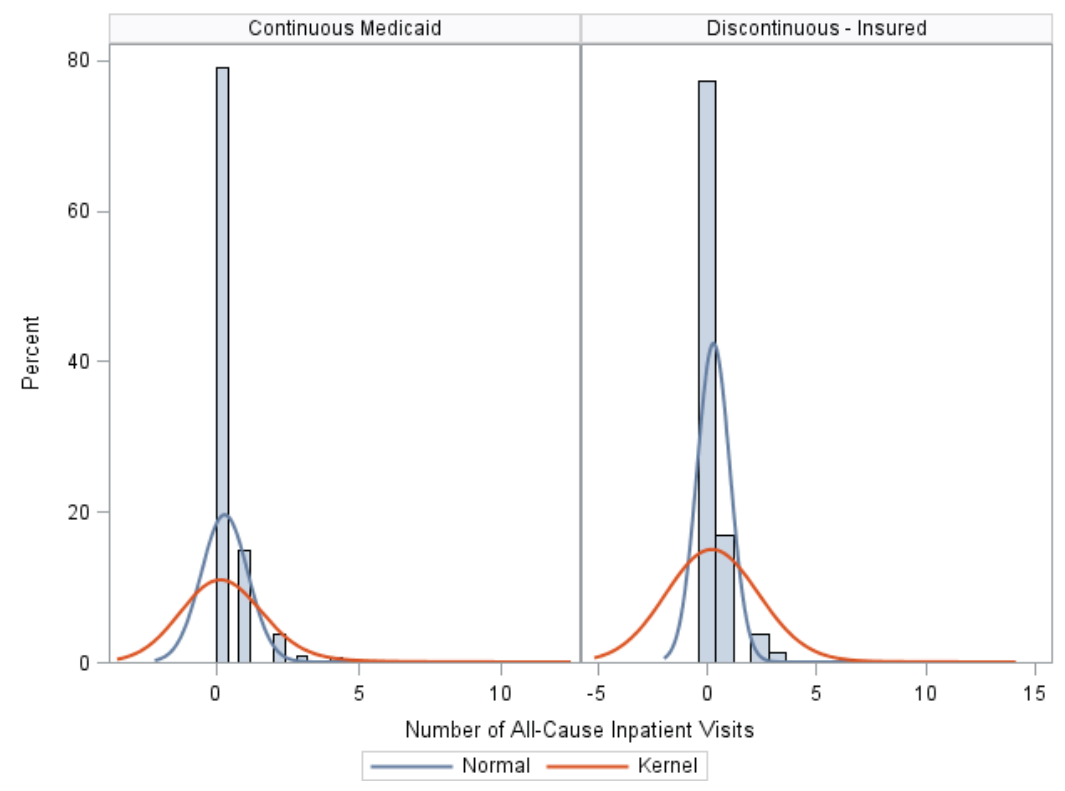

Figure B-2. Distribution of All-Cause Inpatient Visits among Continuous Medicaid and Discontinuous-Insured 


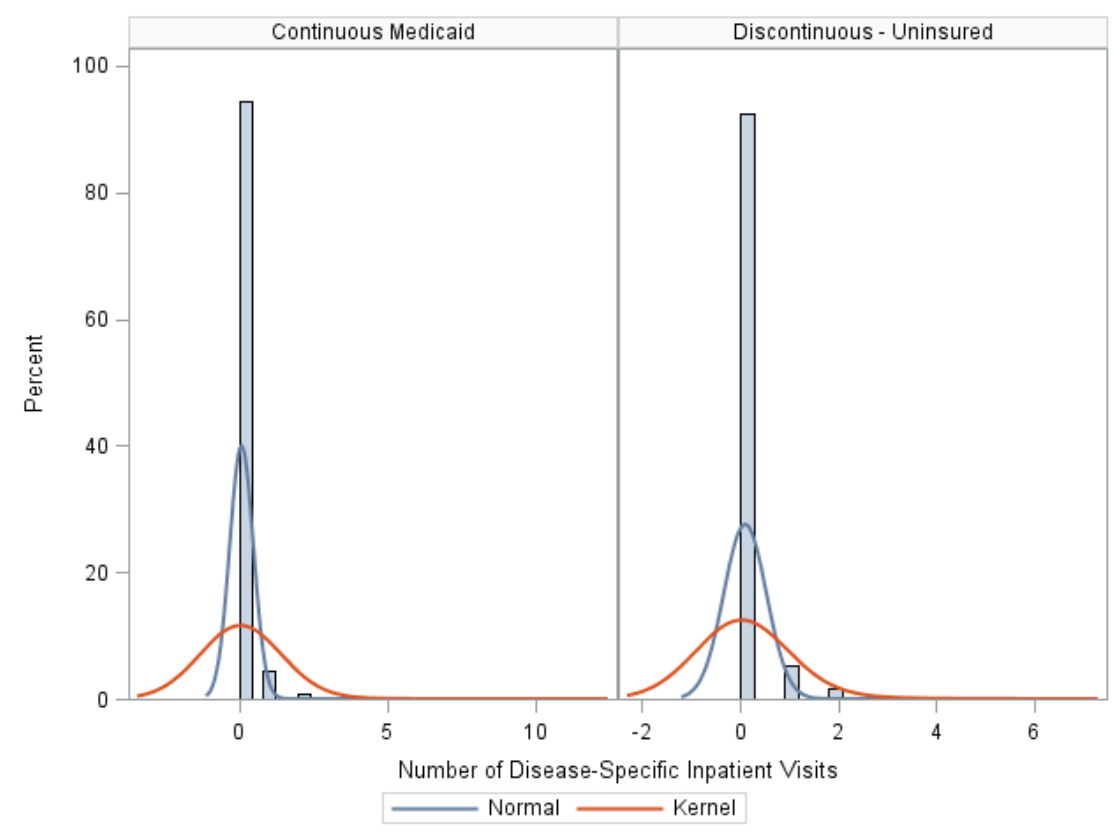

Figure B-3. Distribution of Disease-Specific Inpatient Visits among Continuous Medicaid and Discontinuous-Uninsured

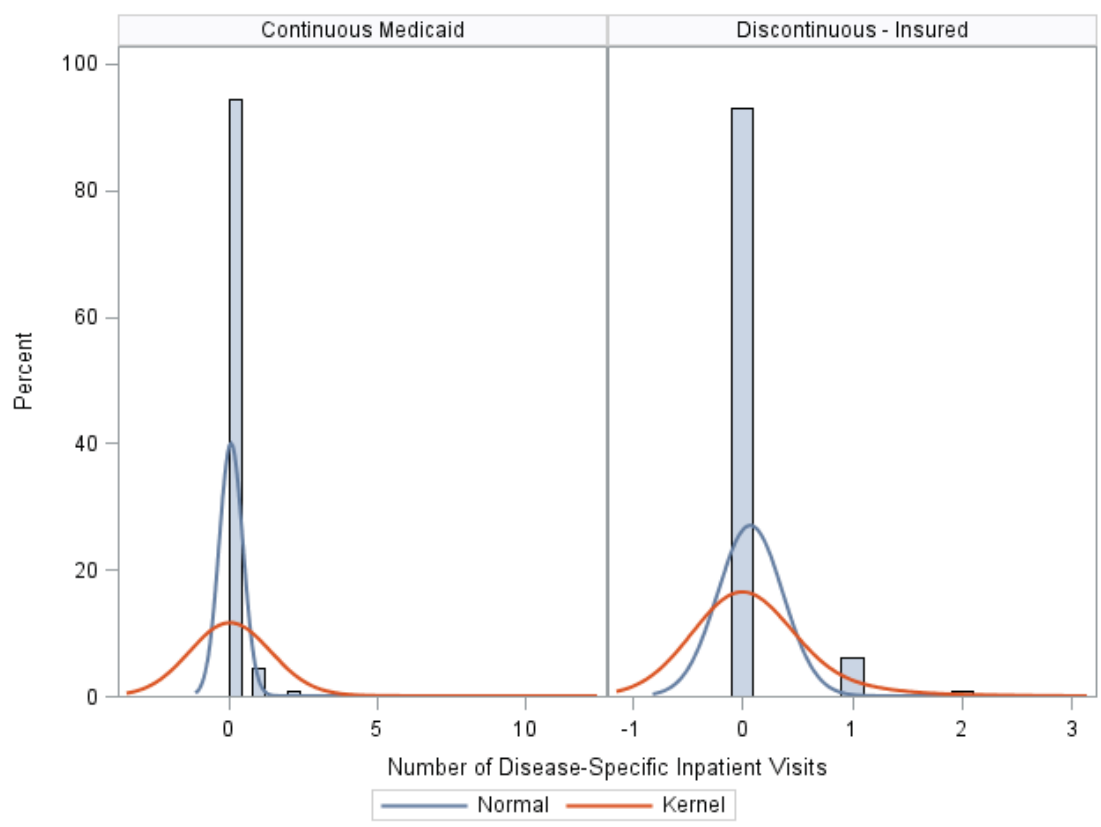

Figure B-4. Distribution of Disease-Specific Inpatient Visits among Continuous Medicaid and Discontinuous-Insured 


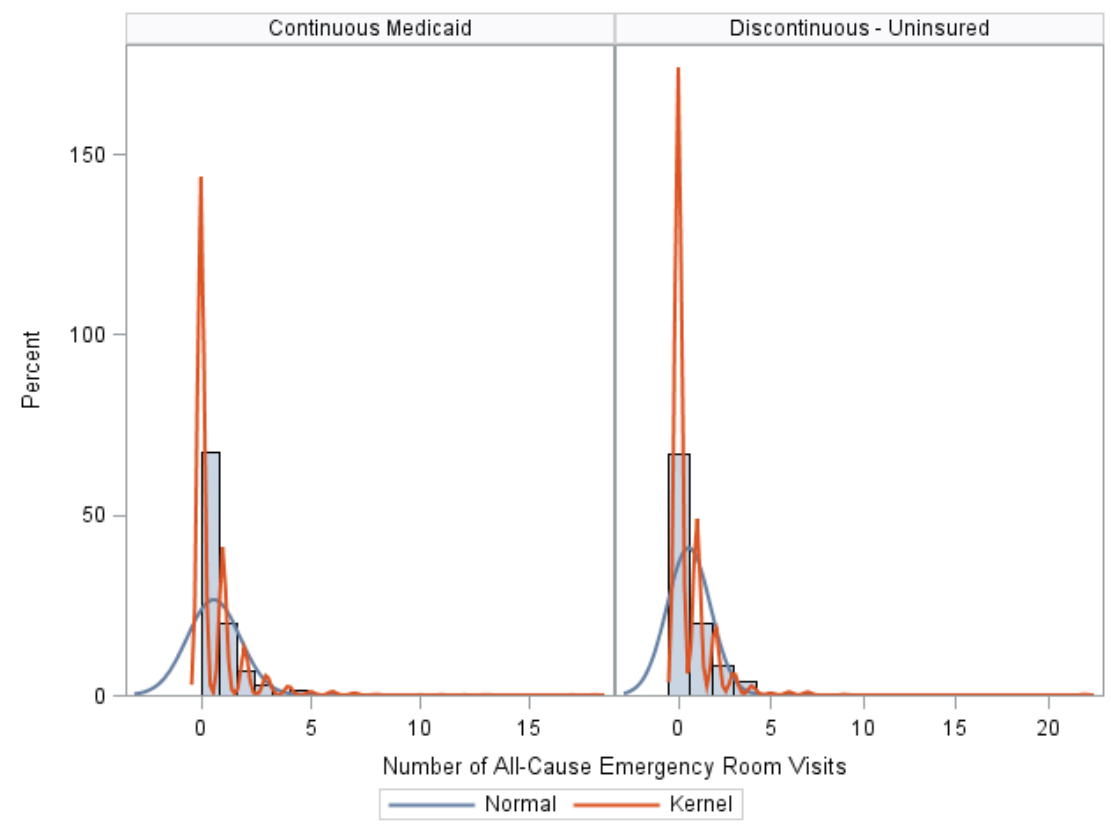

Figure B-5. Distribution of All-Cause Emergency Room Visits among Continuous Medicaid and Discontinuous-Uninsured

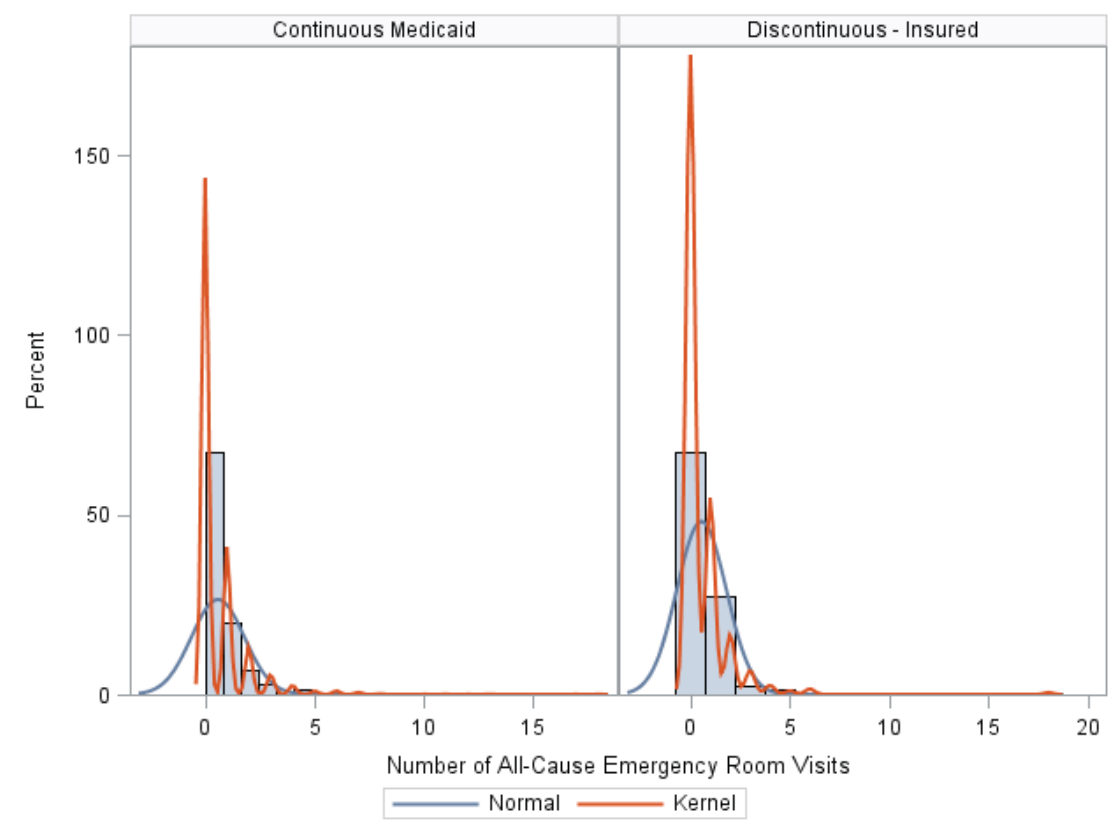

Figure B-6. Distribution of All-Cause Emergency Room Visits among Continuous Medicaid and Discontinuous-Insured 


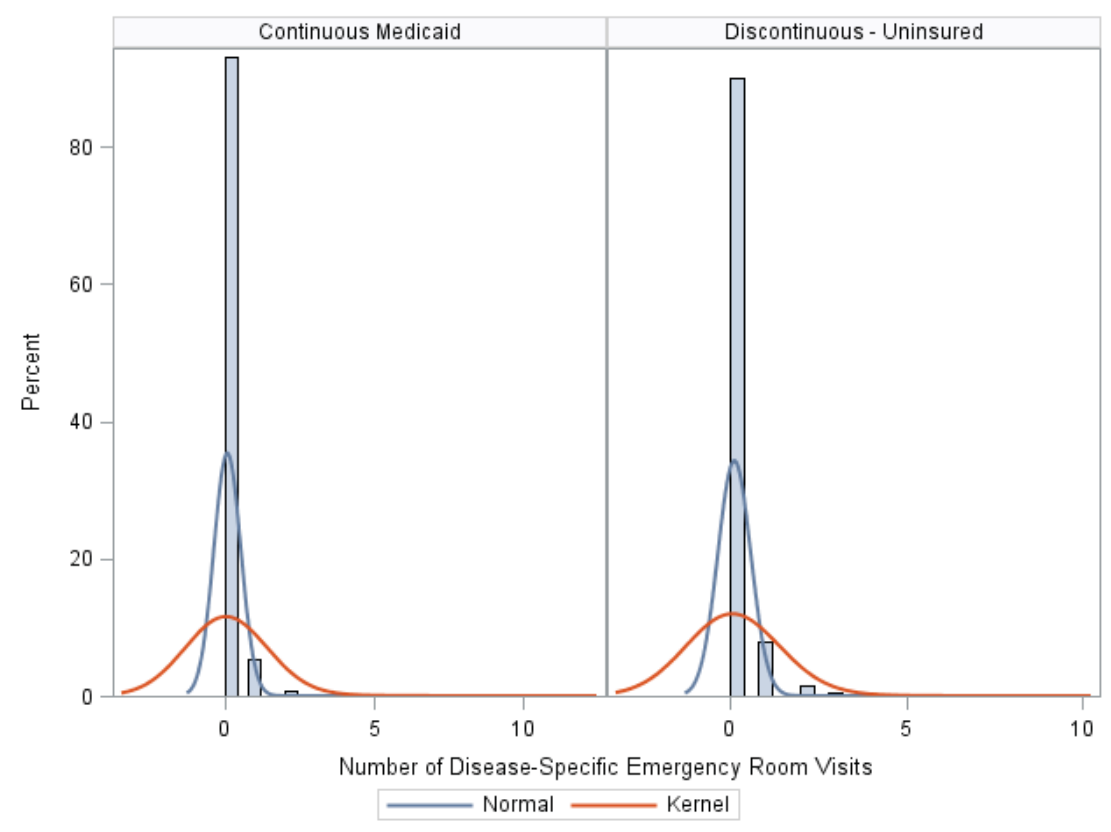

Figure B-7. Distribution of Disease-Specific Emergency Room Visits among Continuous Medicaid and Discontinuous-Uninsured

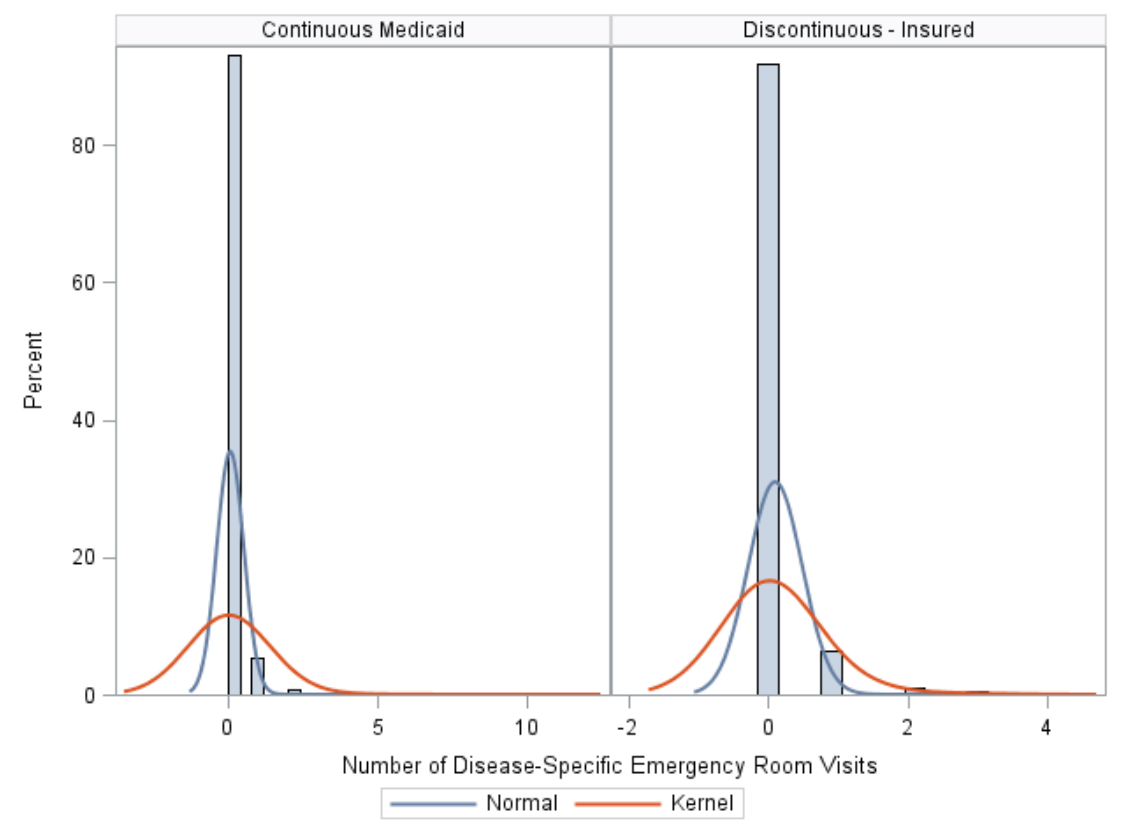

Figure B-8. Distribution of Disease-Specific Emergency Room Visits among Continuous Medicaid and Discontinuous-Insured 


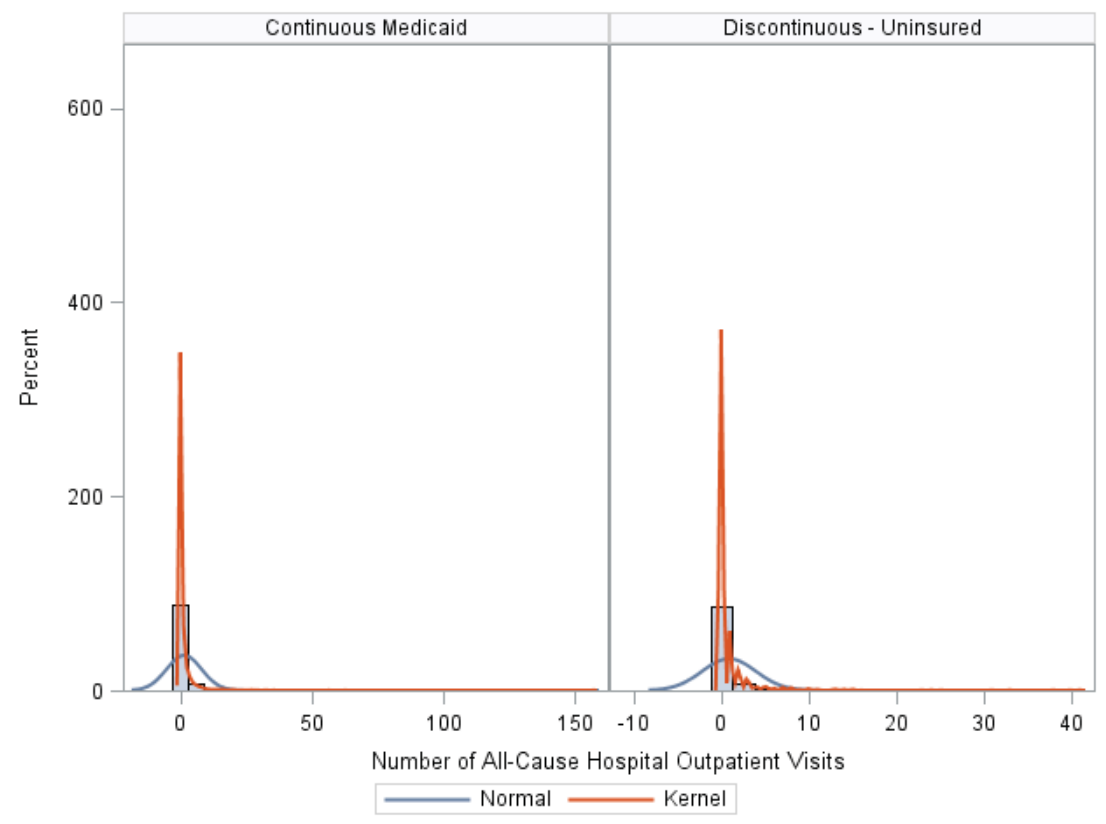

Figure B-9. Distribution of All-Cause Hospital Outpatient Visits among Continuous Medicaid and Discontinuous-Uninsured

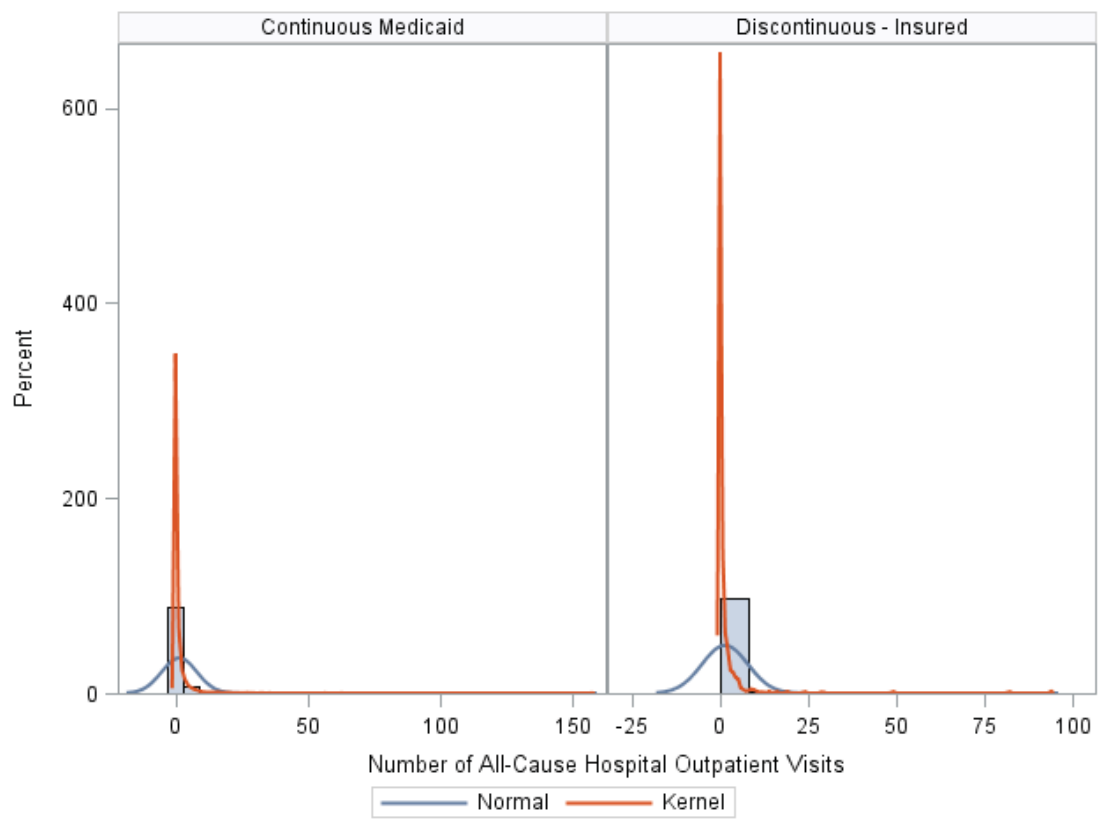

Figure B-10. Distribution of All-Cause Hospital Outpatient Visits among Continuous Medicaid and Discontinuous-Insured 


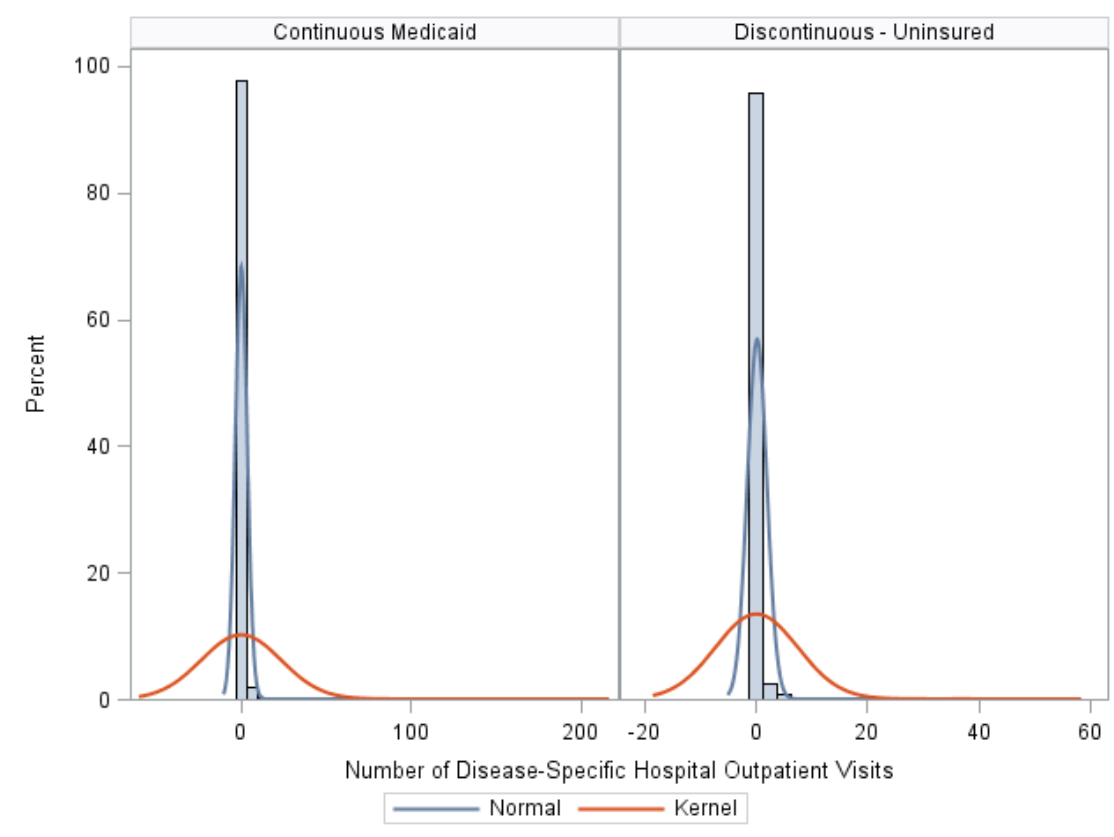

Figure B-11. Distribution of Disease-Specific Hospital Outpatient Visits among Continuous Medicaid and Discontinuous-Uninsured

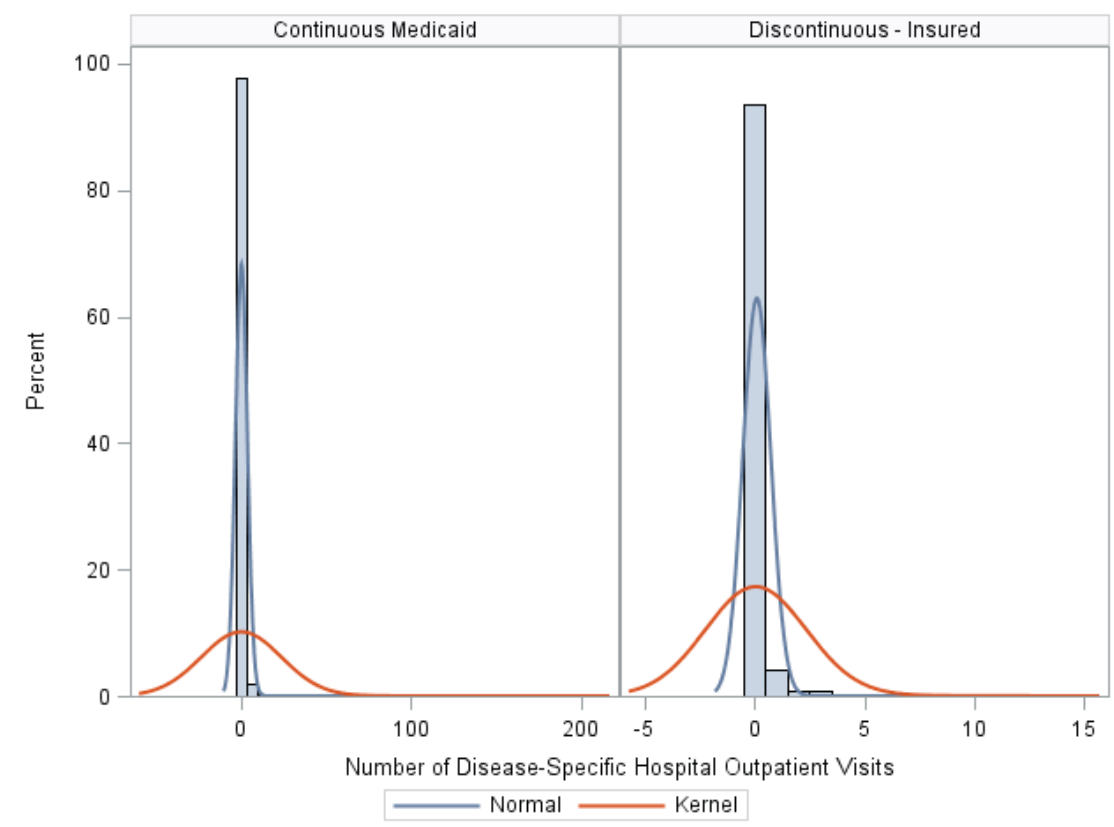

Figure B-12. Distribution of Disease-Specific Hospital Outpatient Visits among Continuous Medicaid and Discontinuous-Insured 


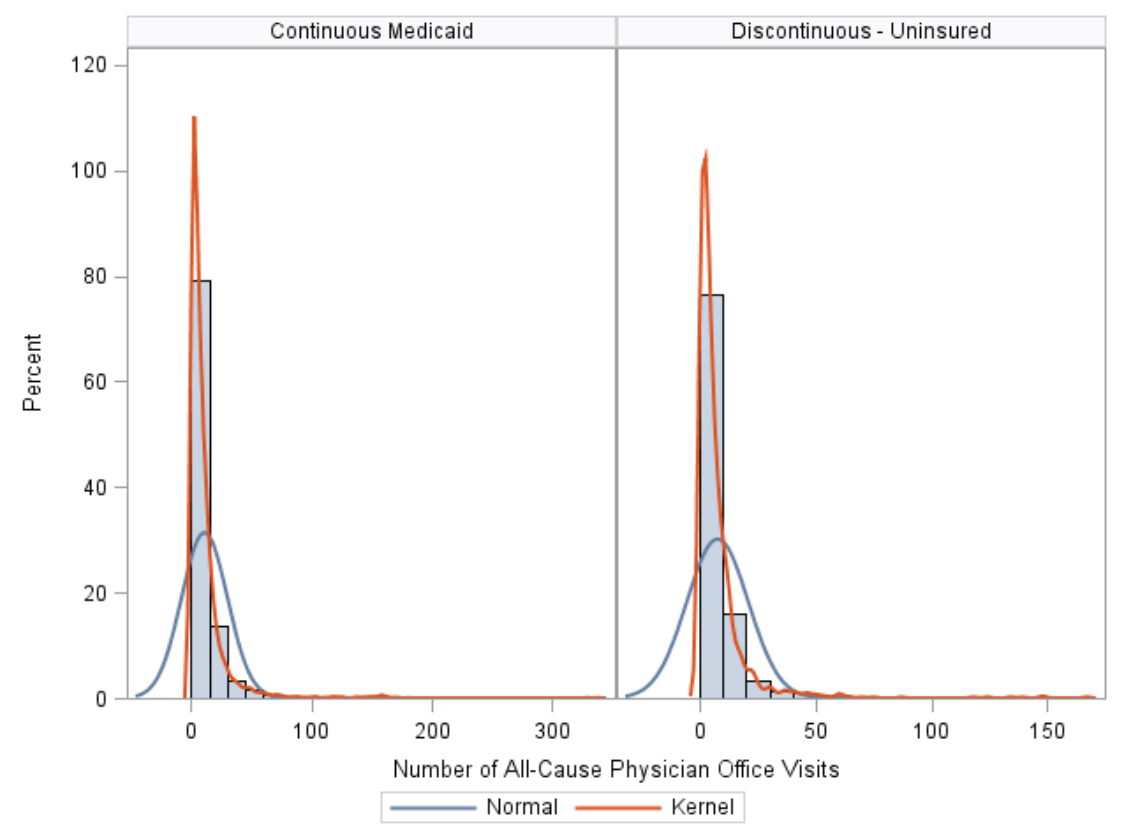

Figure B-13. Distribution of All-Cause Physician Office Visits among Continuous Medicaid and Discontinuous-Uninsured

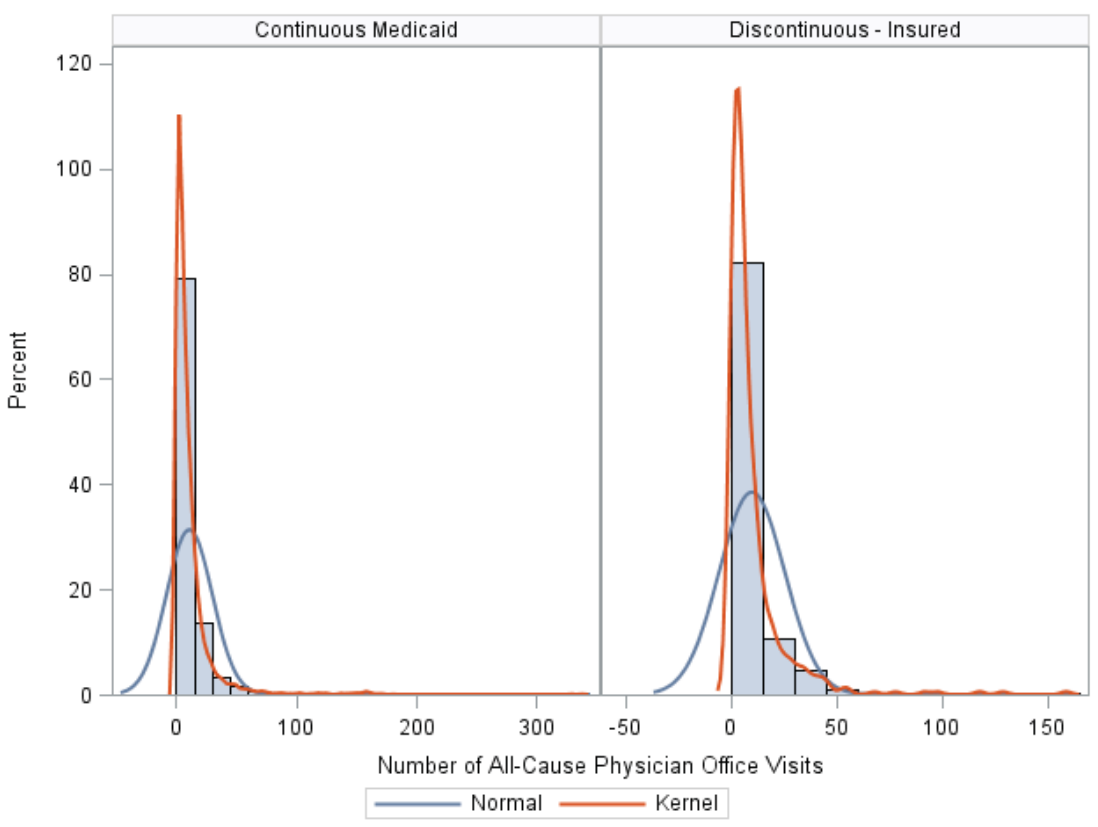

Figure B-14. Distribution of All-Cause Physician Office Visits among Continuous Medicaid and Discontinuous-Insured 


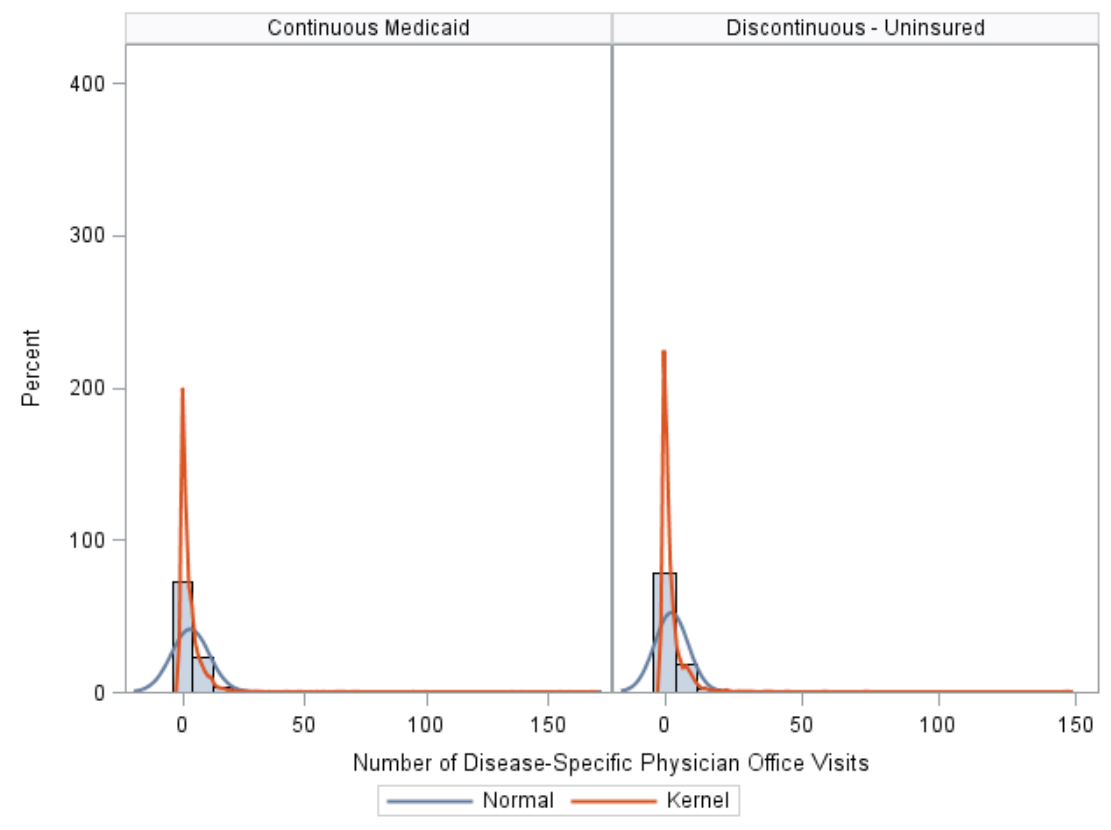

Figure B-15. Distribution of Disease-Specific Physician Office Visits among Continuous Medicaid and Discontinuous-Uninsured

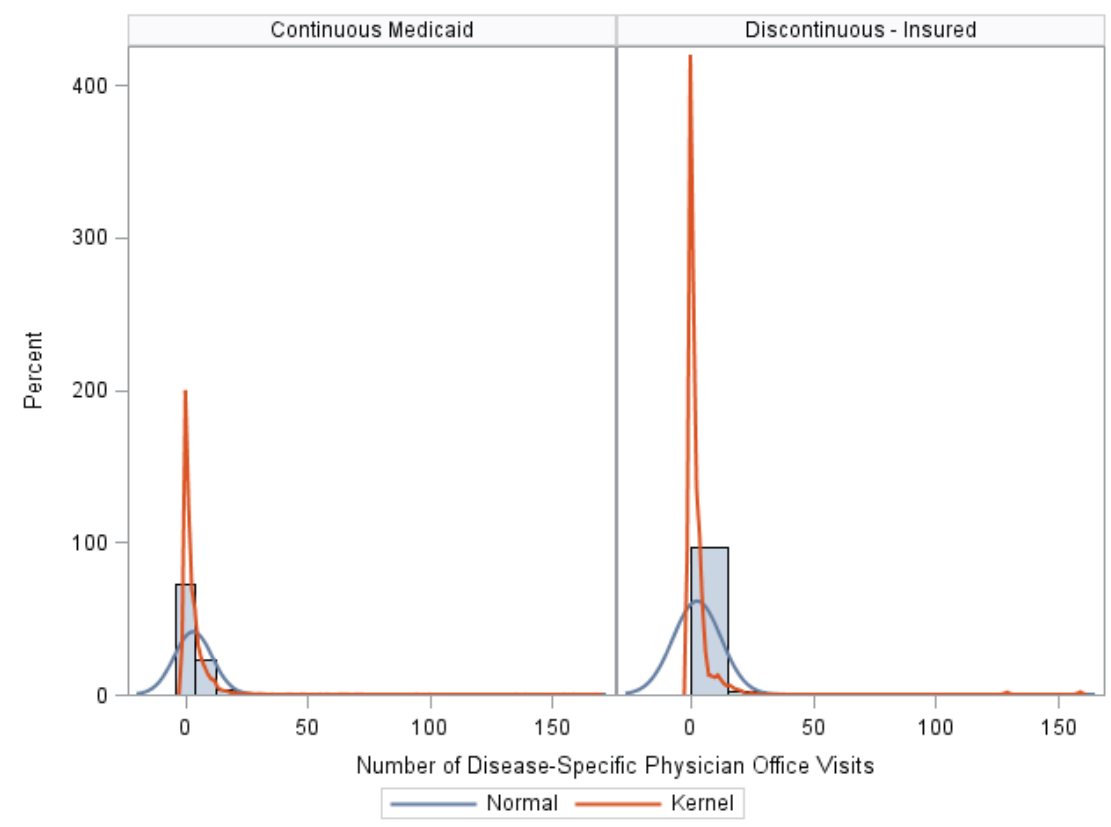

Figure B-16. Distribution of Disease-Specific Physician Office Visits among Continuous Medicaid and Discontinuous-Insured 


\section{VITA}

Kiraat Munshi was born in Mumbai, India in 1984. He is a registered Pharmacist in India after having successfully completed his undergraduate education in Pharmaceutical Sciences from Manipal University in Karnataka, India in 2007. He moved to the United States to pursue his master's degree in Pharmacy Administration at St. John's University in Jamaica, NY, which he successfully obtained in 2010. In 2011 he joined the University of Tennessee Health Science Center (UTHSC) as a doctoral student in the Health Outcomes and Policy Research program, majoring in Health Policy.

During his time at UTHSC, Kiraat has made several presentations, including poster and podium, published papers in peer-reviewed journals, and been in leadership positions in his capacity as the President of the UTHSC Student Chapter of the International Society for Pharmacoeconomics and Outcomes Research (ISPOR) organization, and co-Chair of the ISPOR 2013-2014 Student Council's Education Committee. In his role as a Data Manager for the SafeMed program, Kiraat has successfully handled several responsibilities, including but not limited to, training the SafeMed staff in data entry, oversight of the SafeMed patient data and hospitalization data quality and integrity, periodic analyses of the SafeMed data for examining process measures for the dashboard run charts and quarterly reports, and coordinating with the SafeMed measurement team in obtaining Medicare and Medicaid claims data and designing the final SafeMed data analysis plan. After having successfully defended his dissertation in June 2015, Kiraat will be awarded his doctoral degree in December 2015. 